\title{
Resonant nonlinear Neumann problems with indefinite weight
}

\author{
Dimitri Mugnai And NikolaOs S. Papageorgiou
}

\begin{abstract}
We consider nonlinear Neumann problems driven by the $p$-Laplacian plus an indefinite potential. First we develop the spectral properties of such differential operators. Subsequently, using these spectral properties and variational methods based on critical point theory, truncation techniques and Morse theory, we prove existence and multiplicity theorems for resonant problems.

Mathematics Subject Classification (2010): 35J20 (primary); 35J65, 58E05 (secondary).
\end{abstract}

\section{Introduction}

The aim of this paper is to study the existence and multiplicity of solutions for resonant nonlinear Neumann problems driven by the $p$-Laplacian plus an indefinite potential. To do this, we need to develop the spectral properties of such differential operators. Thus far, such differential operators were used only in the context of Dirichlet boundary value problems. In this direction we mention the works of Cuesta [11], Cuesta-Ramos Quoirin [12], Del Pezzo-Fernandez Bonder [15], Fernandez Bonder-Del Pezzo [16], Leadi-Yechoui [26], Lopez Gomez [32], who deal only with the eigenvalue problem. On the other hand Birindelli-Demengel [7] study Dirichlet problems with the $p$-Laplacian plus an indefinite potential and a superlinear reaction. The study of the corresponding Neumann problems is lagging behind. We mention the recent semilinear (i.e. $p=2$ ) works of Li [27] and Qian [38] and the quasilinear (i.e. $p>1$ ) work of Aizicovici-Papageorgiou-Staicu [1]. However, all the three works consider strictly positive potentials. In $[1,38]$ the reaction is superlinear, while Li [27] considers a reaction which exhibits an oscillatory behaviour.

Our work is divided into two parts. In the first part we deal with the eigenvalue problem and we develop the basic spectral properties of the Neumann $p$-Laplacian plus an indefinite potential, i.e. of eigenvalue problems having the form

$$
\begin{cases}-\Delta_{p} u+\beta|u|^{p-2} u=\hat{\lambda}|u|^{p-2} u & \text { in } \Omega, \\ \frac{\partial u}{\partial n}=0 & \text { on } \partial \Omega\end{cases}
$$

Received December 7, 2010; accepted in revised form March 31, 2011. 
where $\Omega$ is a bounded and smooth domain of $\mathbb{R}^{N}, N \geq 1, \Delta_{p} u=\operatorname{div}\left(|D u|_{N}^{p-2} D u\right)$ is the usual $p$-Laplace differential operator and $\beta$ is a weight function which may change sign.

In the second part of the paper we study asymptotically $p$-linear problems, which may be resonant, and we prove existence and multiplicity results for problems of the form

$$
\begin{cases}-\Delta_{p} u+\beta|u|^{p-2} u=f(z, u) & \text { in } \Omega, \\ \frac{\partial u}{\partial n}=0 & \text { on } \partial \Omega,\end{cases}
$$

where $f: \Omega \times \mathbb{R} \rightarrow \mathbb{R}$ is a function having asymptotically $p$-linear growth at infinity and obeying different hypotheses, according to the different cases we shall study.

Our tools are variational methods based on critical point theory, truncation techniques and Morse theory.

We prove several existence and multiplicity results, allowing the weight to be both unbounded or bounded, according to the conditions we put on the reaction term. The semilinear case, i.e. when $p=2$, can be investigated with the stronger tools of Morse theory, improving the general multiplicity results we get in the general quasilinear case.

For the reader's convenience, in the next section we recall some of the main notions and results from the aforementioned theories that we will use in the sequel.

\section{Mathematical background}

We start by recalling some elements from critical point theory. Let $X$ be a Banach space and let $X^{*}$ be its topological dual. By $\langle\cdot, \cdot\rangle$ we denote the duality brackets for the pair $\left(X^{*}, X\right)$. Let $\phi \in C^{1}(X)$; we say that $\phi$ satisfies the "Palais-Smale condition" ("PS-condition" for short) if the following holds:

every sequence $\left\{x_{n}\right\}_{n \geq 1} \subseteq X$ such that $\left\{\phi\left(x_{n}\right)\right\}_{n \geq 1} \subseteq \mathbb{R}$ is bounded and $\phi^{\prime}\left(x_{n}\right) \rightarrow 0$ in $X^{*}$ as $n \rightarrow \infty$, admits a strongly convergent subsequence.

Sometimes it is more convenient to use a more general compactness condition, known as the "Cerami condition" ("C-condition" for short): we say that $\phi \in C^{1}(\mathbb{R})$ satisfies the C-condition if the following holds:

every sequence $\left\{x_{n}\right\}_{n \geq 1} \subseteq X$ such that $\left\{\phi\left(x_{n}\right)\right\}_{n \geq 1} \subseteq \mathbb{R}$ is bounded and $\left(1+\left\|x_{n}\right\|\right) \phi^{\prime}\left(x_{n}\right) \rightarrow 0$ in $X^{*}$ as $n \rightarrow \infty$, admits a strongly convergent subsequence.

It was shown by Bartolo-Benci-Fortunato [3] that the Deformation Theorem, and consequently the minimax theory of critical values, remains valid if the usual PScondition is replaced by the weaker C-condition.

The following topological notion is important in critical point theory and will be used in the following sections. 
Definition 2.1. Let $Y$ be a Hausdorff topological space and $D_{0}, D$ and $S$ be nonempty closed subsets of $Y$ such that $D_{0} \subseteq D$. We say that the pair $\left\{D_{0}, D\right\}$ is linking with $S$ in $Y$ if and only if

(a) $D_{0} \cap S=\emptyset$;

(b) for all $h \in C(D, Y)$ satisfying $h_{\mid D_{0}}=I d_{\mid D_{0}}$, we have $h(D) \cap S \neq \emptyset$.

Using this notion, we have the following basic minimax principle (see, for example, [18]).

Theorem 2.2. If $X$ is a Banach space, $D_{0}, D$ and $S$ are nonempty closed subsets of $X$, the pair $\left\{D_{0}, D\right\}$ is linking with $S$ in $X, \phi \in C^{1}(\mathbb{R})$ satisfies the $C$-condition,

$$
\sup _{D_{0}} \phi \leq \inf _{S} \phi
$$

set $\Gamma:=\left\{\gamma \in C(D, X): \gamma_{\mid D_{0}}=I d_{\mid D_{0}}\right\}$ and

$$
c:=\inf _{\gamma \in \Gamma} \sup _{u \in D} \phi(\gamma(u))
$$

then $c \geq \inf _{S} \phi$ and $c$ is a critical value for $\phi$. Moreover, if $c=\inf _{S} \phi$, then we can find a critical point of $\phi$ in $S$.

By an appropriate choice of the linking sets, we obtain as corollaries of Theorem 2.2, the Mountain Pass Theorem, the Saddle Point Theorem and the Generalized Mountain Pass Theorem. For future use, we state the Mountain Pass Theorem.

Theorem 2.3. If $X$ is a Banach space, $\phi \in C^{1}(X)$ satisfies the $C$-condition, $x_{0}, x_{1} \in$ $X$ satisfy

$$
\begin{gathered}
\max \left\{\phi\left(x_{0}\right), \phi\left(x_{1}\right)\right\} \leq \inf \left\{\phi(x):\left\|x-x_{0}\right\|=\rho\right\}=\eta_{\rho}, \quad\left\|x_{1}-x_{0}\right\|>\rho, \\
\operatorname{set} \Gamma:=\left\{\gamma \in C([0,1], X): \gamma(0)=x_{0}, \gamma(1)=x_{1}\right\} \text { and } \\
c:=\inf _{\gamma \in \Gamma} \sup _{t \in[0,1]} \phi(\gamma(t)),
\end{gathered}
$$

then $c \geq \eta_{\rho}$ and $c$ is a critical value for $\phi$. Moreover, if $c=\inf _{\partial B_{\rho}\left(x_{0}\right)} \phi$, then we can find a critical point of $\phi$ in $\partial B_{\rho}\left(x_{0}\right)$.

Remark 2.4. It is easy to see that Theorem 2.3 follows from Theorem 2.2 if we choose $D_{0}=\left\{x_{0}, x_{1}\right\}, D=\left[x_{0}, x_{1}\right]=\left\{x \in X: x=(1-t) x_{0}+t x_{1}, 0 \leq t \leq 1\right\}$ and $S=\partial B_{\rho}\left(x_{0}\right)$. 
Next, let us recall some basic definitions and facts from Morse theory which we will use in the sequel. Let $\left(Y_{1}, Y_{2}\right)$ be a topological pair with $Y_{2} \subseteq Y_{1} \subseteq$ $X$. For every integer $k \geq 0$, by $H_{k}\left(Y_{1}, Y_{2}\right)$ we denote the $k^{\text {th }}$-relative singular homology group with integer coefficients for the pair $\left(Y_{1}, Y_{2}\right)$. We also recall that $H_{k}\left(Y_{1}, Y_{2}\right)=0$ for any integer $k<0$. Moreover, if $\phi \in C^{1}(X)$ and $c \in \mathbb{R}$, we introduce the following sets:

$$
\begin{gathered}
\phi^{c}:=\{x \in X: \phi(x) \leq c\}, \quad K_{\phi}:=\left\{x \in X: \phi^{\prime}(x)=0\right\} \\
\text { and } K_{\phi}^{c}=\left\{x \in K_{\phi}: \phi(x)=c\right\} .
\end{gathered}
$$

The critical groups of $\phi$ at an isolated critical point $x \in X$ with $\phi(x)=c$ are defined by

$$
C_{k}(\phi, x):=H_{k}\left(\phi^{c} \cap U, \phi^{c} \cap U \backslash\{x\}\right) \text { for all } k \geq 0,
$$

where $U$ is a neighborhood of $x$ such that $K_{\phi} \cap \phi^{c} \cap U=\{x\}$ (see Chang [9]). The excision property of singular homology theory implies that the previous definition of critical groups is independent of the particular choice of the neighborhood $U$.

Now, suppose that $\phi \in C^{1}(X)$ satisfies the C-condition and that inf $\phi\left(K_{\phi}\right)>$ $-\infty$. Let $c<\phi\left(K_{\phi}\right)$; the critical groups of $\phi$ at infinity are defined by

$$
C_{k}(\phi, \infty):=H_{k}\left(X, \phi^{c}\right) \quad \text { for all } k \geq 0,
$$

see Bartsch-Li [5].

The Deformation Theorem, which is valid since by assumption $\phi$ satisfies the C-condition (see [3]), implies that the definition of critical groups of $\phi$ at infinity is independent of the particular choice of the level $c<\inf \phi\left(K_{\phi}\right)$ used.

If $K_{\phi}$ is finite, defining

$$
M(t, x):=\sum_{k \geq 0} \operatorname{rank} C_{k}(\phi, x) t^{k} \quad \text { for all } t \in \mathbb{R}, x \in K_{\phi}
$$

and

$$
P(t, \infty):=\sum_{k \geq 0} \operatorname{rank} C_{k}(\phi, \infty) t^{k} \quad \text { for all } t \in \mathbb{R},
$$

we have the Morse relation

$$
\sum_{x \in K_{\phi}} M(t, x)=P(t, \infty)+(1+t) Q(t),
$$

where $Q(t):=\sum_{k \geq 0} \beta_{k} t^{k}$ is a formal series in $t \in \mathbb{R}$ with nonnegative integer coefficients (see Chang [9, page 36]).

Now, let $X=H$ be a Hilbert space, $x \in H, U$ is a neighborhood of $x$ and $\phi \in C^{2}(H)$. If $x$ is a critical point of $\phi$, then its "Morse index" is defined to be 
the supremum of the dimensions of the vector subspaces of $H$ on which $\phi^{\prime \prime}(x)$ is negative definite. Also, we say that $x$ is nondegenerate if $\phi^{\prime \prime}(x)$ is invertible. The critical groups of $\phi$ at a nondegenerate critical point $x \in H$ with Morse index $m$ are given by

$$
C_{k}(\phi, x)=\delta_{k, m} \mathbb{Z} \quad \text { for all } k \geq 0,
$$

where $\delta_{k, m}$ is the usual Kronecker delta function

$$
\delta_{k, m}= \begin{cases}1 & \text { if } k=m \\ 0 & \text { if } k \neq m\end{cases}
$$

In this study, we consider a bounded domain $\Omega$ with $C^{2}$ boundary; in addition to the usual Sobolev space $W^{1, p}(\Omega)$, we will also use the following "natural Neumann" spaces:

$$
C_{n}^{1}(\bar{\Omega}):=\left\{u \in C^{1}(\bar{\Omega}): \frac{\partial u}{\partial n}=0 \text { on } \partial \Omega\right\}
$$

and

$$
W_{n}^{1, p}(\Omega):={\overline{C_{n}^{1}(\bar{\Omega})}}^{\|\cdot\|},
$$

where $\|\cdot\|$ denotes the usual norm in $W^{1, p}(\Omega)$. When $p=2$ we also use the notation $H_{n}^{1}(\Omega)$. The Banach space $C_{n}^{1}(\bar{\Omega})$ is an ordered Banach space, with order cone

$$
C_{+}:=\left\{u \in C_{n}^{1}(\bar{\Omega}): u(z) \geq 0 \text { for all } z \in \bar{\Omega}\right\} .
$$

The cone above has a nonempty interior given by

$$
\text { int } C_{+}=\left\{u \in C_{+}: u(z)>0 \text { for all } z \in \bar{\Omega}\right\} .
$$

Another result which we will use is a theorem relating $C_{n}^{1}(\bar{\Omega})-$ and $W_{n}^{1, p}(\Omega)-$ minimizers of a $C^{1}$ functional. More precisely, let $f_{0}: \Omega \times \mathbb{R} \rightarrow \mathbb{R}$ be a Carathéodory function (i.e., the map $z \mapsto f(z, x)$ is measurable for all $x \in \mathbb{R}$ and $x \mapsto f(z, x)$ is continuous for a.e. $z \in \Omega$ ) which has subcritical growth, i.e.

$$
\left|f_{0}(z, x)\right| \leq a_{0}(z)+c_{0}|x|^{r-1} \quad \text { for a.e. } z \in \Omega \text { and all } x \in \mathbb{R} \text {, }
$$

with $a_{0} \in L^{\infty}(\Omega)_{+}=\left\{u \in L^{\infty}(\Omega): u \geq 0\right\}, c_{0}>0$ and

$$
1<r<p^{*}= \begin{cases}\frac{N p}{N-p} & \text { if } p<N \\ \infty & \text { if } p \geq N\end{cases}
$$

Let $F_{0}(z, x)=\int_{0}^{x} f_{0}(z, s) d s$, and consider the $C^{1}$ functional $\psi_{0}: W_{n}^{1, p}(\Omega) \rightarrow \mathbb{R}$ defined by

$$
\psi_{0}(u)=\frac{1}{p}\|D u\|_{p}^{p}-\int_{\Omega} F_{0}(z, u(z)) d z \quad \text { for all } u \in W_{n}^{1, p}(\Omega) .
$$


Concerning $C_{n}^{1}(\bar{\Omega})$ - and $W_{n}^{1, p}(\Omega)$-minimizers for the functional $\psi_{0}$, we have the following result:

Theorem 2.5. If $u_{0} \in W_{n}^{1, p}(\Omega)$ is a local $C_{n}^{1}(\bar{\Omega})$-minimizer of $\psi_{0}$, i.e. there exists $\rho_{0}>0$ such that

$$
\psi_{0}\left(u_{0}\right) \leq \psi_{0}\left(u_{0}+h\right) \quad \text { for all } h \in C_{n}^{1}(\bar{\Omega}) \text { with }\|h\|_{C_{n}^{1}(\bar{\Omega})} \leq \rho_{0},
$$

then $u_{0} \in C_{n}^{1}(\bar{\Omega})$ and $u_{0}$ is also a local $W_{n}^{1, p}(\Omega)$-minimizer of $\psi_{0}$, i.e. there exists $\rho_{1}>0$ such that

$$
\psi_{0}\left(u_{0}\right) \leq \psi_{0}\left(u_{0}+h\right) \quad \text { for all } h \in W_{n}^{1, p}(\Omega) \text { with }\|h\| \leq \rho_{0} .
$$

Remark 2.6. For the "Dirichlet"space $H_{0}^{1}(\Omega)$, this result was first proved by Brezis-Nirenberg [8]. It was extended to the spaces $W_{0}^{1, p}(\Omega)(1<p<\infty)$ by Garcia Azorero-Manfredi-Peral Alonso [17] (see also Guo-Zhang [22] where $p \geq 2$ ). The corresponding result for the "Neumann spaces" $W_{n}^{1, p}(\Omega)(1<p<\infty)$ is due to Motreanu-Motreanu-Papageorgoiu [34].

Let $\langle\cdot, \cdot\rangle$ denote the duality brackets for the pair $\left(W_{n}^{1, p}(\Omega)^{*}, W_{n}^{1, p}(\Omega)\right)$ and consider the nonlinear map $A: W_{n}^{1, p}(\Omega) \rightarrow W_{n}^{1, p}(\Omega)^{*}$ defined by

$$
\langle A(u), v\rangle=\int_{\Omega}|D u|_{N}^{p-2}(D u, D v)_{N} d z \quad \text { for all } u, v \in W_{n}^{1, p}(\Omega) .
$$

Concerning the map $A$, we have the following classical result:

Proposition 2.7. The nonlinear map $A: W_{n}^{1, p}(\Omega) \rightarrow W_{n}^{1, p}(\Omega)^{*}$ defined above is monotone, continuous (hence maximal monotone) and of type $(S)_{+}$, i.e., if $u_{n} \rightarrow u$ in $W_{n}^{1, p}(\Omega)$ and $\lim \sup _{n \rightarrow \infty}\left\langle A\left(u_{n}\right), u_{n}-u\right\rangle \leq 0$, then $u_{n} \rightarrow u$ in $W_{n}^{1, p}(\Omega)$.

As final remarks concerning notations, throughout this work, by $\|\cdot\|$ we will denote the norm in the Sobolev space $W^{1, p}(\Omega)$; as it is already clear from the definition of $\psi_{0}$, by $\|\cdot\|_{p}(1 \leq p \leq \infty)$ we shall denote the norm of $L^{p}(\Omega)$ or of $L^{p}\left(\Omega, \mathbb{R}^{N}\right)$, by $(\cdot, \cdot)_{N}$ and $|\cdot|_{N}$ the scalar product and the norm in $\mathbb{R}^{N}$, respectively, while the Lebesgue measure in $\mathbb{R}^{N}$ will be denoted by $\mathscr{L}_{N}(\cdot)$. Finally, we shall also use the notation $r^{ \pm}=\max \{ \pm r, 0\}$ for any $r \in \mathbb{R}$.

\section{The eigenvalue problem}

In this section we develop the spectral properties of the Neumann $p$-Laplacian plus an indefinite potential. Let $\Omega \subset \mathbb{R}^{N}$ be a bounded domain with a $C^{2}$ boundary $\partial \Omega$. We consider the following nonlinear Neumann eigenvalue problem:

$$
\begin{cases}-\Delta_{p} u+\beta|u|^{p-2} u=\hat{\lambda}|u|^{p-2} u & \text { in } \Omega \\ \frac{\partial u}{\partial n}=0 & \text { on } \partial \Omega\end{cases}
$$


Here $\Delta_{p} u=\operatorname{div}\left(|D u|_{N}^{p-2} D u\right)$ is the $p$-Laplace differential operator and $\beta \in$ $L^{q}(\Omega), \frac{N p}{p-1}<q \leq \infty$, is a weight function which may change sign. By an eigenvalue we mean a real number $\hat{\lambda}$ for which (3.1) has a nontrivial weak solution $u \in W_{n}^{1, p}(\Omega)$. First we show that problem (3.1) has a principal eigenvalue. Note that the weight function $\beta$ can be both indefinite (i.e. changes sign) and unbounded. We mention that problem (3.1) was studied for $N=1$ (ordinary differential equations), using completely different methods by Zhang [43] and Binding-Rynne [6].

Proposition 3.1. If $\beta \in L^{q}(\Omega)$ with $q>\frac{N p}{p-1}$, then problem (3.1) has a smallest eigenvalue $\hat{\lambda}_{1} \in \mathbb{R}$ which is simple and has a positive eigenfunction $\hat{u}_{1} \in C^{1, \alpha}(\Omega)$ with $0<\alpha<1$.

Proof. Let $\mathscr{E}: W^{1, p}(\Omega) \rightarrow \mathbb{R}$ be the $C^{1}$ functional defined by

$$
\mathscr{E}(u)=\|D u\|_{p}^{p}+\int_{\Omega} \beta|u|^{p} d z,
$$

and consider the set $M \subset W^{1, p}(\Omega)$ defined by

$$
M=\left\{u \in W^{1, p}(\Omega):\|u\|_{p}=1\right\} .
$$

By Liusternik's Theorem (see, for example, [36], page 74) we know that $M$ is a $C^{1}$ Banach manifold. Let

$$
\hat{\lambda}_{1}:=\inf \{\mathscr{E}(u): u \in M\} .
$$

Claim 1: $\hat{\lambda}_{1}>-\infty$.

Note that $q>\frac{N p}{p-1}=N p^{\prime}\left(p^{\prime}\right.$ being the conjugate exponent of $p$, i.e. $\left.\frac{1}{p}+\frac{1}{p^{\prime}}=1\right)$, hence $q^{\prime}<\left(N p^{\prime}\right)^{\prime}=\frac{N p}{N p-p+1}$, and so we have $p q^{\prime}<p^{*}$. Then, if $u \in W^{1, p}(\Omega)$, by the Sobolev Embedding Theorem, we have $|u|^{p} \in L^{q^{\prime}}(\Omega)$. Hence, by virtue of Hölder's inequality, we have

$$
\left.\left|\int_{\Omega} \beta\right| u\right|^{p} d z \mid \leq\|\beta\|_{q}\|u\|_{p q^{\prime}}^{p} .
$$

We know that $W^{1, p}(\Omega) \hookrightarrow L^{p q^{\prime}}(\Omega) \hookrightarrow L^{p}(\Omega)$ and the first embedding is compact. So, invoking Ehrling's inequality (see, for example, [36], page 698), given $\varepsilon>0$, we can find $c(\varepsilon)>0$ such that

$$
\|u\|_{p q^{\prime}} \leq \varepsilon\|u\|+c(\varepsilon)\|u\|_{p}^{p} \quad \text { for all } u \in W^{1, p}(\Omega) .
$$

From (3.3) and (3.4) we have

$$
\|D u\|_{p}^{p}+\|u\|_{p}^{p}-\int_{\Omega} \beta|u|^{p} d z \leq\|D u\|_{p}^{p}+\|u\|_{p}^{p}+\varepsilon\|\beta\|_{q}\|u\|^{p}+c(\varepsilon) \beta\left\|_{q}\right\| u \|_{p}^{p},
$$


so that

$$
\left(1-\varepsilon\|\beta\|_{q}\right)\|u\|^{p} \leq \mathscr{E}(u)+\left(1+c(\varepsilon)\|\beta\|_{q}\right)\|u\|_{p}^{p} .
$$

We now choose $\varepsilon \in\left(0,1 /\|\beta\|_{q}\right)$. Then

$$
\hat{c}(\varepsilon)\|u\|^{p} \leq \mathscr{E}(u)+\left(1+c(\varepsilon)\|\beta\|_{q}\right)\|u\|_{p}^{p}, \quad \text { with } \hat{c}(\varepsilon):=c(\varepsilon)-\varepsilon\|\beta\|_{q}>0 .
$$

So, if $u \in M$, then from (3.5) we see that

$$
-\left(1+c(\varepsilon)\|\beta\|_{q}\right) \leq \mathscr{E}(u),
$$

and thus $\hat{\lambda}_{1}>-\infty$, which proves Claim 1 .

Claim 2: The infimum in (3.2) is realized at a function $\hat{u}_{1} \in M$.

Let $\left\{u_{n}\right\}_{n \geq 1} \subset M$ be a minimizing sequence, i.e. $\mathscr{E}\left(u_{n}\right) \rightarrow \lambda_{1}^{+}$as $n \rightarrow \infty$. It is clear from (3.5) that $\left\{u_{n}\right\}_{n \geq 1} \subset W^{1, p}(\Omega)$ is bounded. So, up to a subsequence, we may assume that

$$
u_{n} \rightarrow \hat{u}_{1} \text { in } W^{1, p}(\Omega) \text { and } u_{n} \rightarrow \hat{u}_{1} \text { in } L^{p q^{\prime}}(\Omega) \text { as } n \rightarrow \infty .
$$

From (3.6) we infer that

$$
\left\|D \hat{u}_{1}\right\|_{p}^{p} \leq \liminf _{n \rightarrow \infty}\left\|D u_{n}\right\|_{p}^{p} \quad \text { and } \quad \lim _{n \rightarrow \infty} \int_{\Omega} \beta\left|u_{n}\right|^{p} d z=\int_{\Omega} \beta\left|\hat{u}_{1}\right|^{p} d z,
$$

and thus $\mathscr{E}\left(\hat{u}_{1}\right) \leq \lambda_{1}$.

It is clear from (3.6) that $\left\|\hat{u}_{1}\right\|_{p}=1$, i.e. $\hat{u}_{1} \in M$. Hence $\mathscr{E}\left(\hat{u}_{1}\right)=\hat{\lambda}_{1}$, and this proves Claim 2.

The Lagrange multiplier rule (see, for example, [36, page 76], implies that $\hat{\lambda}_{1}$ is an eigenvalue for problem (3.1), with $\hat{u}_{1} \in W^{1, p}(\Omega)$ being the associated eigenfunction. An application of the Moser iteration technique (see, for example, Hu-Papageorgiou [23]) implies that $\hat{u}_{1} \in L^{\infty}(\Omega)$. Then, invoking Theorem 4.4 of Le [25], we infer that $\hat{u}_{1} \in C^{1, \alpha}(\Omega)$ for some $\alpha \in(0,1)$. Moreover, since $\mathscr{E}(|u|)=\mathscr{E}(u)$, we deduce that $\hat{u}_{1}$ does not change sign and we may assume that $\hat{u}_{1} \geq 0$. Then, using the Harnack inequality of Trudinger [41], we conclude that $\hat{u}_{1}(z)>0$ for all $z \in \Omega$.

To show the simplicity of $\hat{\lambda}_{1}$, we use the generalized Picone identity due to Allegretto-Huang [2]. Let $\hat{v}_{1} \in W^{1, p}(\Omega)$ be another eigenfunction associated to $\hat{\lambda}_{1}$. From the previous discussion we know that $\hat{v}_{1} \in C^{1, \alpha}(\Omega)$ and we may also assume that $\hat{v}_{1}(z)>0$ for all $z \in \Omega$. Now set $\hat{v}_{1}^{n}:=\hat{v}_{1}+1 / n, n \geq 1$, and introduce the following functions defined in $\Omega$ :

$$
\begin{gathered}
L\left(\hat{u}_{1}, \hat{v}_{1}^{n}\right):=\left|D \hat{u}_{1}\right|_{N}^{p}+(p-1) \frac{\hat{u}_{1}^{p}}{\left(\hat{v}_{1}^{n}\right)^{p}}\left|D \hat{v}_{1}^{n}\right|_{N}^{p}-p \frac{\hat{u}_{1}^{p}}{\left(\hat{v}_{1}^{n}\right)^{p}}\left|D \hat{v}_{1}^{n}\right|_{N}^{p-2}\left(D \hat{v}_{1}^{n}, D \hat{u}_{1}\right)_{N}, \\
R\left(\hat{u}_{1}, \hat{v}_{1}^{n}\right):=\left|D \hat{u}_{1}\right|_{N}^{p}-\left|D \hat{v}_{1}^{n}\right|_{N}^{p-2}\left(D\left(\frac{\hat{u}_{1}^{p}}{\left(\hat{v}_{1}^{n}\right)^{p-1}}\right), D \hat{v}_{1}^{n}\right)_{N} .
\end{gathered}
$$


From the generalized Picone identity of Allegretto-Huang [2] (see also [36, page 328], we have

$$
\begin{aligned}
0 & \leq \int_{\Omega} L\left(\hat{u}_{1}, \hat{v}_{1}^{n}\right) d z=\int_{\Omega} R\left(\hat{u}_{1}, \hat{v}_{1}^{n}\right) d z \\
& =\int_{\Omega}\left[\left|D \hat{u}_{1}\right|_{N}^{p}-\left|D \hat{v}_{1}^{n}\right|_{N}^{p-2}\left(D \hat{v}_{1}^{n}, D\left(\frac{\hat{u}_{1}^{p}}{\left(\hat{v}_{1}^{n}\right)^{p-1}}\right)\right)_{N}\right] d z ;
\end{aligned}
$$

since $\hat{u}_{1}^{p} / \hat{v}_{1}^{n} \in W^{1, p}(\Omega)$, by the nonlinear Green's identity (see [36, page 330], the last integral equals

$$
\int_{\Omega}\left[\left|D \hat{u}_{1}\right|_{N}^{p}+\Delta_{p} \hat{v}_{1}^{n} \frac{\hat{u}_{1}^{p}}{\left(\hat{v}_{1}^{n}\right)^{p-1}}\right] d z=\int_{\Omega}\left[\left|D \hat{u}_{1}\right|_{N}^{p}+\Delta_{p} \hat{v}_{1} \frac{\hat{u}_{1}^{p}}{\left(\hat{v}_{1}^{n}\right)^{p-1}}\right] d z .
$$

Since $\hat{v}_{1}$ solves (3.1), then the last integral is equal to

$$
\int_{\Omega}\left[\left|D \hat{u}_{1}\right|_{N}^{p}+\left(\beta-\hat{\lambda}_{1}\right) \hat{v}_{1}^{p-1} \frac{\hat{u}_{1}^{p}}{\left(\hat{v}_{1}^{n}\right)^{p-1}}\right] d z .
$$

By the Lebesgue Dominated Convergence Theorem, passing to the limit, we finally get

$$
0 \leq \int_{\Omega}\left[\left|D \hat{u}_{1}\right|_{N}^{p}+\beta \hat{u}_{1}^{p}\right] d z-\hat{\lambda}_{1}\left\|\hat{u}_{1}\right\|_{p}^{p}=\mathscr{E}\left(\hat{u}_{1}\right)-\hat{\lambda}_{1},
$$

since $\hat{u}_{1} \in M$. This clearly implies that $\int_{\Omega} L\left(\hat{u}_{1}, \hat{v}_{1}\right) d z=0$, and since $L\left(\hat{u}_{1}, \hat{v}_{1}\right) \geq 0$ (see $[2,36])$, then

$$
L\left(\hat{u}_{1}(z), \hat{v}_{1}(z)\right)=0 \quad \text { for a.e. } z \in \Omega,
$$

which implies that there exists $k>0$ such that

$$
\hat{u}_{1}=k \hat{v}_{1} \text {, }
$$

see $[2,36]$.

This proves that $\hat{\lambda}_{1}$ is simple, i.e. $\hat{\lambda}_{1}$ is a principal eigenvalue.

If $\beta \in L^{\infty}(\Omega)$ (bounded weight function), then we can improve the conclusion of the previous proposition.

Proposition 3.2. If $\beta \in L^{\infty}(\Omega)$, then problem (3.1) has a smallest eigenvalue $\hat{\lambda}_{1} \in \mathbb{R}$ which is simple and has an eigenfunction $\hat{u}_{1} \in \operatorname{int} C_{+} \cap C^{1, \alpha}(\bar{\Omega})$ for some $\alpha \in(0,1]$.

Proof. The existence and simplicity of the smallest eigenvalue $\hat{\lambda}_{1} \in \mathbb{R}$ follows from Proposition 3.1, and again we have $\hat{u}_{1} \in L^{\infty}(\Omega)$. Since $\beta \in L^{\infty}(\Omega)$, we can apply Theorem 2 of Lieberman [29] and conclude that $\hat{u}_{1} \in C^{1, \alpha}(\bar{\Omega})$ for some $\alpha \in(0,1)$, and that $\hat{u}_{1} \geq 0$. Note that

$$
\Delta_{p} \hat{u}_{1} \leq\left(\|\beta\|_{\infty}+\hat{\lambda}_{1}\right) \hat{u}_{1}^{p-1} \quad \text { a.e. in } \Omega,
$$

and thus $\hat{u}_{1} \in \operatorname{int} C_{+}$, see Vazquez [42] and Pucci-Serrin [37, page 120]. 
Remark 3.3. In (3.1) we can admit a weighted right hand side of the form $\tilde{\lambda} m(z)|u(z)|^{p-2} u(z)$ with $m \in L^{\infty}(\Omega), m \geq 0, m \not \equiv 0$. Indeed, as above, we have a principal eigenvalue $\tilde{\lambda}_{1}(m) \in \mathbb{R}$. Moreover, $m \mapsto \tilde{\lambda}_{1}(m)$ is continuous on $L^{\infty}\left(\tilde{\sim}_{)_{+}}\right.$and exhibits the following monotonicity property: if $m \leq m^{\prime}, m \neq \equiv m^{\prime}$, then $\tilde{\lambda}_{1}\left(m^{\prime}\right)<\tilde{\lambda}_{1}(m)$, see (3.2) and Proposition 3.1.

Next we show that any eigenfunction corresponding to an eigenvalue $\lambda \neq \hat{\lambda}_{1}$ is nodal (i.e. sign changing) and that $\hat{\lambda}_{1}$ is isolated.

Proposition 3.4. If $\beta \in L^{q}(\Omega), q>\frac{N p}{p-1}$, then every eigenvalue $\lambda \neq \hat{\lambda}_{1}$ has nodal eigenfunctions and $\hat{\lambda}_{1}$ is isolated.

Proof. Let $\lambda$ be an eigenvalue different from $\hat{\lambda}_{1}$ and let $u \in W^{1, p}(\Omega)$ be an eigenfunction associated to it. We know that $u \in C^{1, \alpha}(\Omega)$ for some $\alpha \in(0,1]$ (see Le [25]). Suppose that $u$ is not nodal. Then, without loss of generality, we may assume that $u \geq 0$. As before, using the Harnack inequality of Trudinger [41] (see also Pucci-Serrin [37, page 163]), we have $u(z)>0$ for every $z \in \Omega$. Set $u^{\varepsilon}=u+\varepsilon, \varepsilon>0$. Then, as in the proof of Proposition 3.1, using the generalized Picone identity, we have

$$
\begin{aligned}
& 0 \leq \Omega L\left(\hat{u}_{1}, u^{\varepsilon}\right) d z=\int_{\Omega} R\left(\hat{u}_{1}, u^{\varepsilon}\right) d z \\
& \hat{\lambda}_{1}-\int_{\Omega} \beta \hat{u}_{1}^{p} d z+\int_{\Omega} \beta u^{p-1} \frac{\hat{u}_{1}^{p}}{(u+\varepsilon)^{p-1}} d z-\lambda \int_{\Omega} u^{p-1} \frac{\hat{u}_{1}^{p}}{(u+\varepsilon)^{p-1}} d z .
\end{aligned}
$$

By the Lebesgue Dominated Convergence Theorem this implies

$$
0 \leq\left(\hat{\lambda}_{1}-\lambda\right) \int_{\Omega} \hat{u}_{1}^{p} d z=\hat{\lambda}_{1}-\lambda<0
$$

since $\lambda>\hat{\lambda}_{1}$. This is a contradiction, thus proving that the eigenfunction $u$ corresponding to $\lambda$ must be nodal.

Now, let $D \subseteq \Omega$ be a nodal domain of $u$ (i.e., a connected component of $\Omega \backslash\{u=0\})$. We know that $u \chi_{D} \in W^{1, p}(\Omega)$ and $D\left(u \chi_{D}\right)=(D u) \chi_{D}, \chi_{D}$ being the characteristic function of the set $D$. We also have

$$
A(u)+\beta|u|^{p-2} u=\lambda|u|^{p-2} u .
$$

Acting on (3.7) with $h=u \chi_{D}$, we obtain

$$
\left\langle A(u), u \chi_{D}\right\rangle+\int_{D} \beta|u|^{p} d z=\lambda \int_{D}|u|^{p} d z
$$

that is, since $D\left(u \chi_{D}\right)=(D u) \chi_{D}$,

$$
\int_{D}|D u|_{N}^{p} d z+\int_{D} \beta|u|^{p} d z=\lambda \int_{D}|u|^{p} d z .
$$


Hence

$$
\mathscr{E}\left(u \chi_{D}\right)=\lambda \int_{D}|u|^{p} d z
$$

From the proof of Proposition 3.1 (see (3.5)), we know that there exists $c_{1}>0$ such that

$$
\begin{aligned}
\left\|u \chi_{D}\right\|^{p} & \leq c_{1}\left(\mathscr{E}\left(u \chi_{D}\right)+\left\|u \chi_{D}\right\|_{p}^{p}\right) \\
& =c_{1}(\lambda+1)\left\|u \chi_{D}\right\|_{p}^{p} \quad \text { by }(3.8) \\
& \leq c_{1}(\lambda+1) \mathscr{L}_{N}(D)^{1-p / p^{*}}\left\|u \chi_{D}\right\|_{p^{*}}^{p},
\end{aligned}
$$

since $p<p^{*}$ and so $L^{p^{*}}(\Omega) \hookrightarrow L^{p}(\Omega)$. Thus, by the Sobolev Embedding Theorem, there exists $c_{2}>0$ such that

$$
\left\|u \chi_{D}\right\|_{p^{*}}^{p} \leq c_{2} \mathscr{L}_{N}(D)^{1-p / p^{*}}\left\|u \chi_{D}\right\|_{p^{*}}^{p},
$$

and hence, since $u \neq 0$,

$$
1 \leq c_{2} \mathscr{L}_{N}(D)^{1-p / p^{*}}
$$

Using this estimate, we can prove that the principal eigenvalue $\hat{\lambda}_{1}$ is isolated. We argue by contradiction: suppose that we can find a sequence $\left\{\lambda_{n}\right\}_{n>1} \subset \mathbb{R}$ such that $\lambda_{n} \rightarrow \hat{\lambda}_{1}^{+}$and $\lambda_{n}$ is an eigenvalue for problem (3.1) for every $n \geq 1$. Then there exist $u_{n} \in W^{1, p}(\Omega) \cap C^{1}(\Omega) \backslash\{0\}$ such that

$$
A\left(u_{n}\right)+\beta\left|u_{n}\right|^{p-2} u_{n}=\lambda_{n}\left|u_{n}\right|^{p-2} u_{n} \quad \forall n \geq 1 .
$$

Let $y_{n}=u_{n} /\left\|u_{n}\right\|, n \geq 1$. Then $\left\|y_{n}\right\|=1$ for all $n \geq 1$, and so we can assume that

$$
y_{n} \rightarrow y \text { in } W^{1, p}(\Omega) \text { and } y_{n} \rightarrow y \text { in } L^{p q^{\prime}}(\Omega) \text { and a.e. in } \Omega \text { as } n \rightarrow \infty \text {. }
$$

Multiplying (3.10) by $\left\|u_{n}\right\|^{1-p}$, we obtain

$$
A\left(y_{n}\right)+\beta\left|y_{n}\right|^{p-2} y_{n}=\lambda_{n}\left|y_{n}\right|^{p-2} y_{n} \quad \text { for all } n \geq 1 \text {. }
$$

Acting on (3.12) with $y-y_{n} \in W^{1, p}(\Omega)$, passing to the limit as $n \rightarrow \infty$ and using (3.11), we obtain

$$
\lim _{n \rightarrow \infty}\left\langle A\left(y_{n}, y_{n}-y\right\rangle=0,\right.
$$

which implies, by Proposition 2.7 , that

$$
y_{n} \rightarrow y \text { in } W^{1, p}(\Omega) \text { and a.e. in } \Omega .
$$

Therefore, if in (3.12) we pass to the limit as $n \rightarrow \infty$ and use (3.13), we have

$$
A(y)+\beta|y|^{p-2} y=\hat{\lambda}_{1}|y|^{p-2} y \quad \text { with }\|y\|=1 \text { by (3.13). }
$$

Hence

$$
\mathscr{E}(y)=\hat{\lambda}_{1}\|y\|_{p}^{p},
$$

and so, by Proposition 3.1, there exists $k \in \mathbb{R} \backslash\{0\}$ such that $y=k \hat{u}_{1}$. 
Without any loss of generality, we may assume that $k>0$. Now, let $D_{-}^{n}=$ $\left\{z \in \Omega: y_{n}(z)<0\right\}$. Since $\hat{u}_{1}>0$ in $\Omega$, we have $\mathscr{L}_{N}\left(D_{-}^{n}\right) \rightarrow 0$ as $n \rightarrow \infty$, which, together with (3.13), contradicts (3.9). Therefore, we conclude that $\hat{\lambda}_{1}$ is isolated.

Denote by $\sigma_{p}(\Omega)$ the set of all eigenvalues for problem (3.1). It is clear from the proof of Proposition 3.4 above, that the following result holds.

Proposition 3.5. If $\beta \in L^{q}(\Omega)$ with $q>\frac{N p}{p-1}$, then the set $\sigma_{p}(\Omega) \subset \mathbb{R}$ of eigenvalues of (3.1) is closed.

From Propositions 3.4 and 3.5 it follows that

$$
\hat{\lambda}_{2}=\inf \left\{\lambda: \lambda \in \sigma_{p}(\Omega) \backslash\left\{\hat{\lambda}_{1}\right\}\right\} \in \sigma_{p}(\Omega),
$$

and this is the second eigenvalue for problem (3.1). For this second eigenvalue, we have the following minimax characterization.

Proposition 3.6. If $\beta \in L^{q}(\Omega)$ with $q>\frac{N p}{p-1}$, then

$$
\hat{\lambda}_{2}=\inf _{\gamma \in \Gamma} \max _{-1 \leq t \leq 1} \mathscr{E}(\gamma(t)),
$$

where $\Gamma=\left\{\gamma \in C([-1,1], M): \gamma(-1)=-\hat{u}_{1}, \gamma(1)=\hat{u}_{1}\right\}$.

Proof. First we show that $\mathscr{E}$ satisfies the PS-condition on the manifold $M$ : let $\left\{u_{n}\right\}_{n \geq 1} \subseteq M$ be such that

$$
\left|\mathscr{E}\left(u_{n}\right)\right| \leq M_{1} \quad \text { for some } M_{1}>0 \text { and for all } n \geq 1
$$

and

$$
\left.\left|\left\langle A\left(u_{n}\right), h\right\rangle+\int_{\Omega} \beta\right| u_{n}\right|^{p-2} u_{n} h d z \mid \leq \varepsilon_{n}\|h\| \quad \text { for all } h \in T_{u_{n}} M,
$$

where $\varepsilon_{n} \rightarrow 0^{+}$as $n \rightarrow \infty$. Here, as usual, $T_{u_{n}} M$ denotes the tangent space to $M$ at $u_{n}$. We know that

$$
T_{u_{n}} M=\left\{h \in W^{1, p}(\Omega): \int_{\Omega}\left|u_{n}\right|^{p-2} u_{n} h d z=0\right\}, \quad n \geq 1,
$$

for example see [18, page 600]. Then, for every $y \in W^{1, p}(\Omega)$, we have that

$$
h=y-\left(\int_{\Omega}\left|u_{n}\right|^{p-2} u_{n} y d z\right) u_{n} \in T_{u_{n}} M \quad \text { for all } n \geq 1 .
$$


Using this $h$ as a test function in (3.15), we obtain

$$
\left.\left|\left\langle A\left(u_{n}\right), y\right\rangle+\int_{\Omega} \beta\right| u_{n}\right|^{p-2} u_{n} y d z-\left(\int_{\Omega}\left|u_{n}\right|^{p-2} u_{n} y d z\right) \mathscr{E}\left(u_{n}\right) \mid \leq \varepsilon_{n}\|h\|
$$

for all $n \geq 1$. By [20, page 48], there exists $c_{3}>0$ such that

$$
\left.\left|\left\langle A\left(u_{n}\right), y\right\rangle+\int_{\Omega} \beta\right| u_{n}\right|^{p-2} u_{n} y d z-\left(\int_{\Omega}\left|u_{n}\right|^{p-2} u_{n} y d z\right) \mathscr{E}\left(u_{n}\right) \mid \leq \varepsilon_{n} c_{3}\|y\|
$$

for all $n \geq 1$.

From the proof of Proposition 3.1, see (3.5), we know that there exists $c_{4}>0$ such that

$$
\left\|u_{n}\right\|^{p} \leq c_{4}\left(\mathscr{E}\left(u_{n}\right)+1\right) \quad \text { for all } n \geq 1 .
$$

Hence, by (3.14), we get that $\left\{u_{n}\right\}_{n \geq 1} \subseteq W^{1, p}(\Omega)$ is bounded. Thus we may assume that

$$
u_{n} \rightarrow u \text { in } W^{1, p}(\Omega) \text { and } u_{n} \rightarrow u \text { in } L^{p q^{\prime}}(\Omega) .
$$

In (3.16) we choose $y=u_{n}-u \in W^{1, p}(\Omega)$, pass to the limit as $n \rightarrow \infty$ and use (3.17); in this way we obtain

$$
\lim _{n \rightarrow \infty}\left\langle A\left(u_{n}\right), u_{n}-u\right\rangle=0,
$$

which, together with Proposition 2.7, implies that

$$
u_{n} \rightarrow u \text { in } W^{1, p}(\Omega) \text { as } n \rightarrow \infty .
$$

This proves that $\mathscr{E}$ satisfies the PS-condition on the $C^{1}$ Banach manifold $M$.

Now, we know that $\pm \hat{u}_{1}$ are both minimizers of $\mathscr{E}$ with $\mathscr{E}\left( \pm \hat{u}_{1}\right)=\hat{\lambda}_{1}$. Moreover, it is clear that both $\pm \hat{u}_{1}$ are strict local minimizers of $\mathscr{E}$. More precisely, we will now show that for $\rho_{0}>0$ small enough, we can find $\rho_{ \pm} \in\left(0, \rho_{0}\right)$ such that

$$
\mathscr{E}\left( \pm \hat{u}_{1}\right)<\inf \left\{\mathscr{E}(u): u \in M \text { and }\left\|u-\left( \pm \hat{u}_{1}\right)\right\|=\rho_{ \pm}\right\} \quad \text { and } \rho_{ \pm}<2\left\|\hat{u}_{1}\right\|
$$

We do the proof for $\hat{u}_{1}$, the proof being analogous for $-\hat{u}_{1}$. Arguing by contradiction, suppose that, given any $\rho \in\left(0, \rho_{0}\right)$, we can find a sequence $\left\{u_{n}\right\}_{n \geq 1} \subseteq$ $M \cap \partial B_{\rho}\left(\hat{u}_{1}\right)$ such that $\mathscr{E}\left(u_{n}\right) \rightarrow \mathscr{E}\left(\hat{u}_{1}\right)$ as $n \rightarrow \infty$. By (3.5) it is readily seen that $\left\{u_{n}\right\}_{n \geq 1} \subseteq W^{1, p}(\Omega)$ is bounded, thus we may assume that

$$
u_{n} \rightarrow u \text { in } W^{1, p}(\Omega) \text { and } u_{n} \rightarrow u \text { in } L^{p q^{\prime}}(\Omega) .
$$

Since $\mathscr{E}$ is sequentially weakly lower semicontinuous, from (3.19) it immediately follows that

$$
\mathscr{E}(u) \leq \liminf _{n \rightarrow \infty} \mathscr{E}\left(u_{n}\right)=\mathscr{E}\left(\hat{u}_{1}\right)=\hat{\lambda}_{1}
$$


By (3.19) we get that $u \in M$; hence from $\mathscr{E}(u)=\mathscr{E}\left(\hat{u}_{1}\right)=\hat{\lambda}_{1}$ we get $u=\hat{u}_{1}$ by Proposition 3.1. By the Mean Value Theorem we can find $t_{n} \in(0,1)$ such that

$$
\begin{aligned}
\mathscr{E}\left(u_{n}\right)- & \mathscr{E}\left(\frac{u_{n}+\hat{u}_{1}}{2}\right)=\left\langle\mathscr{E}^{\prime}\left(t_{n} u_{n}+\left(1-t_{n}\right) \frac{u_{n}+\hat{u}_{1}}{2}\right), \frac{u_{n}-\hat{u}_{1}}{2}\right\rangle \\
= & \left\langle A\left(t_{n} u_{n}+\left(1-t_{n}\right) \frac{u_{n}+\hat{u}_{1}}{2}\right), \frac{u_{n}-\hat{u}_{1}}{2}\right\rangle \\
& +\int_{\Omega} \beta\left|t_{n} u_{n}+\left(1-t_{n}\right) \frac{u_{n}+\hat{u}_{1}}{2}\right|^{p-2}\left(t_{n} u_{n}+\left(1-t_{n}\right) \frac{u_{n}+\hat{u}_{1}}{2}\right) \frac{u_{n}-\hat{u}_{1}}{2} d z .
\end{aligned}
$$

Assuming without loss of generality that $t_{n} \rightarrow t \in[0,1]$, we pass to the limit as $n \rightarrow \infty$ in the previous equality: from (3.19), the sequential weak lower semicontinuity of $\mathscr{E}$, the convergence $\mathscr{E}\left(u_{n}\right) \rightarrow \mathscr{E}\left(\hat{u}_{1}\right)$, since $t_{n}+1>0$ and recalling that $u=\hat{u}_{1}$, we obtain

$$
\limsup _{n \rightarrow \infty} \frac{t_{n}+1}{2}\left\langle A\left(t_{n} u_{n}+\left(1-t_{n}\right) \frac{u_{n}+\hat{u}_{1}}{2}\right), u_{n}-\hat{u}_{1}\right\rangle \leq 0,
$$

and thus

$$
\limsup _{n \rightarrow \infty}\left\langle A\left(t_{n} u_{n}+\left(1-t_{n}\right) \frac{u_{n}+\hat{u}_{1}}{2}\right), t_{n} u_{n}+\left(1-t_{n}\right) \frac{u_{n}+\hat{u}_{1}}{2}-\hat{u}_{1}\right\rangle \leq 0,
$$

which implies by Proposition 2.7 that

$$
t_{n} u_{n}+\left(1-t_{n}\right) \frac{u_{n}+\hat{u}_{1}}{2} \rightarrow \hat{u}_{1} \quad \text { in } W^{1, p}(\Omega) .
$$

But note that

$$
0 \leftarrow\left\|t_{n} u_{n}+\left(1-t_{n}\right) \frac{u_{n}+\hat{u}_{1}}{2}-\hat{u}_{1}\right\|=\frac{t_{n}+1}{2}\left\|u_{n}-\hat{u}_{1}\right\| \geq \frac{\rho}{2} \quad \text { for all } n \geq 1,
$$

since $t_{n}+1>1$, a contradiction. Similarly for $-\hat{u}_{1}$ and these two things together prove (3.18).

It follows that

$$
\tilde{\lambda}=\inf _{\gamma \in \Gamma} \max _{-1 \leq t \leq 1} \mathscr{E}(\gamma(t))>\mathscr{E}\left( \pm \hat{u}_{1}\right)=\hat{\lambda}_{1}
$$

since every path joining $\hat{u}_{1}$ and $-\hat{u}_{1}$ necessarily crosses $\partial B_{\rho_{ \pm}}\left( \pm \hat{u}_{1}\right)$. Since $\mathscr{E} \mid M$ satisfies the PS-condition, we can apply Corollary 3.6 of Ghoussoub [19, page 53-54] and infer that $\tilde{\lambda}$ is a critical value of $\mathscr{E} \mid M$, and thus an eigenvalue for problem(3.1) with $\tilde{\lambda}>\hat{\lambda}_{1}$.

We will now show that $\tilde{\lambda}=\lambda_{2}$ by showing that there is no eigenvalue of (3.1) contained in the open interval $\left(\hat{\lambda}_{1}, \tilde{\lambda}\right)$. We argue indirectly: suppose by contradiction that $\lambda \in\left(\lambda_{1}, \tilde{\lambda}\right)$ is an eigenvalue of (3.1) and let $u \in W^{1, p}(\Omega)$ be an 
eigenfunction associated to $\lambda$. From Proposition 3.4 we know that $u$ is nodal, and so both $u^{+} \neq 0$ and $u^{-} \neq 0$. Now consider the following two paths on the manifold $M$ :

$$
\begin{aligned}
& \left.\gamma_{1}(t)=\frac{u^{+}-t u^{-}}{\left\|u^{+}-t u^{-}\right\|_{p}}, \quad t \in[0,1] \quad \text { (this path joins } \frac{u^{+}}{\left\|u^{+}\right\|_{p}} \text { and } u\right) \\
& \left.\gamma_{2}(t)=\frac{-u^{-}+(1-t) u^{+}}{\left\|-u^{-}+(1-t) u^{+}\right\|_{p}}, \quad t \in[0,1] \quad \text { (this path joins } u \text { and } \frac{-u^{-}}{\left\|u^{-}\right\|_{p}}\right)
\end{aligned}
$$

The concatenation of the two paths $\gamma=\gamma_{1} \cup \gamma_{2}$ joins $\frac{u^{+}}{\left\|u^{+}\right\|_{p}}$ and $\frac{-u^{-}}{\left\|u^{-}\right\|_{p}}$. Moreover, note that the functions $u^{+}$and $u^{-}$have disjoint interior supports, and since $A(u)+\beta|u|^{p-2} u=\lambda|u|^{p-2} u$, acting first with $u^{+} \in W^{1, p}(\Omega)$ and then with $u^{-} \in W^{1, p}(\Omega)$, we obtain

$$
\mathscr{E}\left(u^{+}\right)=\lambda\left\|u^{+}\right\|_{p}^{p} \text { and } \mathscr{E}\left(u^{-}\right)=\lambda\left\|u^{-}\right\|_{p}^{p}
$$

Therefore, it easily follows that

$$
\mathscr{E}\left(\gamma_{1}(t)\right)=\mathscr{E}\left(\gamma_{2}(t)\right)=\lambda \quad \text { for all } t \in[0,1] .
$$

We now consider the set $\hat{L}_{2}=\left\{u \in W^{1, p}(\Omega): \mathscr{E}(u)<\lambda\right\}$. Evidently, $\hat{L}_{2}$ is not path-connected, or otherwise we could violate the minimax expression of $\tilde{\lambda}>\lambda$. Moreover, using the Ekeland variational principle and using the fact that $\mathscr{E}_{\mid M}$ satisfies the PS-condition, we infer that every path component of $\hat{L}_{2}$ contains a critical point of $\mathscr{E}_{\mid M}$. Since the only critical points of $\mathscr{E}_{\mid M}$ in $\hat{L}_{2}$ are $\pm \hat{u}_{1}$ and, as already observed, $\hat{L}_{2}$ is not path-connected, we infer that $\hat{L}_{2}$ has exactly two path components, one containing $\hat{u}_{1}$, and the other containing $-\hat{u}_{1}$.

Note that $\frac{u^{+}}{\left\|u^{+}\right\|_{p}}$ cannot be a critical point of $\mathscr{E}_{\mid M}$, since $\frac{u^{+}}{\left\|u^{+}\right\|_{p}}$ has constant sign and $\mathscr{E}\left(\frac{u^{+}}{\left\|u^{+}\right\|_{p}}\right)=\lambda$. So we can find a path $s:[-\varepsilon, \varepsilon] \rightarrow M$ such that

$$
s(0)=\frac{u^{+}}{\left\|u^{+}\right\|_{p}} \quad \text { and } \quad \frac{d}{d t}\left(\mathscr{E}_{\mid M}\right)(s(t)) \neq 0 .
$$

Hence, starting with this path, we can move from $\frac{u^{+}}{\left\|u^{+}\right\|_{p}}$ to a point $y \in M$ along a path on the manifold $M$ which stays within $\hat{L}_{2}$, with the exception of the starting point $\frac{u^{+}}{\left\|u^{+}\right\|_{p}}$. So $u \in \hat{L}_{2}$, and we denote by $U_{1}$ the path-component of $\hat{L}_{2}$ containing $y$. Without any loss of generality, we may assume that $\hat{u}_{1} \in U_{1}$. Then we can join $y$ and $\hat{u}_{1}$ with a path remaining in $U_{1}$. Concatenating such a path with the path $s$, we see that finally we have a path $\gamma_{+}:[0,1] \rightarrow \overline{U_{1}}$ such that

$$
\gamma_{+}(0)=\hat{u}_{1}, \gamma_{+}(1)=\frac{u^{+}}{\left\|u^{+}\right\|_{p}} \text { and } \gamma_{+}(t) \in U_{1} \subseteq \hat{L}_{2} \text { for all } t \in[0,1) .
$$


In a similar fashion, if $U_{2}$ is the other path-component of $\hat{L}_{2}$ with $-\hat{u}_{1} \in U_{2}$, we can produce a path $\gamma_{-}:[0,1] \rightarrow \overline{U_{2}}$ such that

$$
\gamma_{-}(0)=\frac{-u^{-}}{\left\|u^{-}\right\|_{p}}, \gamma_{-}(1)=-\hat{u}_{1} \text { and } \gamma_{-}(t) \in U_{2} \subseteq \hat{L}_{2} \text { for all } t \in(0,1] \text {. }
$$

Concatenating $\gamma_{+}, \gamma, \gamma_{-}$, we produce a path $\gamma_{*} \in \Gamma$ such that $\mathscr{E}\left(\gamma_{*}(t)\right) \leq \lambda$ for all $t \in[-1,1]$; hence $\tilde{\lambda} \leq \lambda$, a contradiction with the fact that $\lambda<\tilde{\lambda}$. Therefore, there is no eigenvalue of (3.1) in the interval $\left(\hat{\lambda}_{1}, \tilde{\lambda}\right)$, and so we conclude that $\tilde{\lambda}=\hat{\lambda}_{2}$.

Remark 3.7. In fact from the above proof we see that we also have the characterization

$$
\hat{\lambda}_{2}=\inf _{\hat{\gamma} \in \hat{\Gamma}} \max _{-1 \leq t \leq 1} \mathscr{E}(\hat{\gamma}(t)) \text {, }
$$

where

$$
\hat{\Gamma}=\{\hat{\gamma} \in C([-1,1], M): \hat{\gamma}(-1) \leq 0 \leq \hat{\gamma}(1)\} .
$$

Now, let $V=\left\{u \in W^{1, p}(\Omega): \int_{\Omega} \hat{u}_{1} u d z=0\right\}$ and set

$$
\hat{\lambda}_{V}=\inf \{\mathscr{E}(u): u \in M \cap V\}
$$

Proposition 3.8. $\hat{\lambda}_{1}<\hat{\lambda}_{V} \leq \hat{\lambda}_{2}$.

Proof. First of all, it is clear from (3.20) that $\hat{\lambda}_{1} \leq \hat{\lambda}_{V}$.

Suppose by contradiction that $\hat{\lambda}_{1}=\hat{\lambda}_{V}$. Then we can find a sequence $\left\{u_{n}\right\}_{n \geq 1} \subset$ $M \cap V$ such that $\mathscr{E}\left(u_{n}\right) \rightarrow \hat{\lambda}_{1}=\hat{\lambda}_{V}$. By (3.5) we immediately see that $\left\{u_{n}\right\}_{n \geq 1} \subset$ $W^{1, p}(\Omega)$ is bounded, and so we may assume that

$$
u_{n} \rightarrow u \text { in } W^{1, p}(\Omega) \text { and } u_{n} \rightarrow u \text { in } L^{p q^{\prime}}(\Omega)
$$

Exploiting the sequential weak lower semicontinuity of $\mathscr{E}$, by (3.21) and since $u \in$ $M \cap V$, we have

$$
\hat{\lambda}_{1} \leq \mathscr{E}(u) \leq \liminf _{n \rightarrow \infty} \mathscr{E}\left(u_{n}\right)=\hat{\lambda}_{1}=\hat{\lambda}_{V}
$$

and hence

$$
\mathscr{E}(u)=\hat{\lambda}_{1},
$$

which implies that $u= \pm \hat{u}_{1}$, a contradiction to the fact that $u \in M \cap V$.

Thus $\hat{\lambda}_{1}<\hat{\lambda}_{V}$.

Now, suppose that $\hat{\lambda}_{2}<\hat{\lambda}_{V}$. Then, by virtue of Proposition 3.6, we can find $\gamma \in \Gamma$ such that

$$
\mathscr{E}(\gamma(t))<\hat{\lambda}_{V} \quad \text { for all } t \in[-1,1]
$$


From the very definition of $\Gamma$, we know that $\gamma(-1)=-\hat{u}_{1}$ and $\gamma(1)=\hat{u}_{1}$. So, if we consider the continuous function $\sigma:[-1,1] \rightarrow \mathbb{R}$ defined by

$$
\sigma(t)=\int_{\Omega} \hat{u}_{1} \gamma(t) d z \quad \text { for all } t \in[-1,1],
$$

then $\sigma(-1)<0<\sigma(1)$, and so by Bolzano's Theorem on intermediate values, we can find $t^{*} \in(-1,1)$ such that $\sigma\left(t^{*}\right)=0$. Hence $\gamma\left(t^{*}\right) \in V$ and so $\hat{\lambda}_{V} \leq \mathscr{E}\left(\gamma\left(t^{*}\right)\right)$ by (3.20), which contradicts (3.22).

Remark 3.9. Let $\mathcal{S}=\left\{V \subseteq W^{1, p}(\Omega): W^{1, p}(\Omega)=\mathbb{R} \oplus V\right\}$, i.e. $\mathcal{S}$ is the set of all topological complements of $\mathbb{R}$ in $W^{1, p}(\Omega)$ and set

$$
\hat{\lambda}_{\mathcal{S}}=\inf _{V \in \mathcal{S}} \sup _{v \in V} \mathscr{E}(v) .
$$

A natural question is wether $\hat{\lambda}_{\mathcal{S}}=\hat{\lambda}_{2}$.

\section{Nonlinear problems: existence theorems}

In this section we use the spectral properties established in Section 3, in order to study the existence of nontrivial smooth solutions for the following nonlinear Neumann problem with indefinite weight:

$$
\begin{cases}-\Delta_{p} u+\beta|u|^{p-2} u=f(z, u) & \text { in } \Omega \\ \frac{\partial u}{\partial n}=0 & \text { on } \partial \Omega\end{cases}
$$

First we prove an existence theorem for resonant (hence noncoercive) equations. The hypotheses on the reaction function $f$ are the following:

$\boldsymbol{H}_{\mathbf{1}}: f: \Omega \times \mathbb{R} \rightarrow \mathbb{R}$ is a Carathéodory function such that $f(z, 0)=0$ a.e. in $\Omega$ and

(i): there exist $a \in L^{\infty}(\Omega)_{+}, c>0$ such that $|f(z, x)| \leq a(z)+c|x|^{p-1}$ for a.e. $z \in \Omega$ and all $x \in \mathbb{R}$

(ii): there exists $\eta_{0}<\hat{\lambda}_{2}, \beta_{0}>0$ and $\tau \in[1, p]$ such that, if $F(z, x)=$ $\int_{0}^{x} f(z, s) d s$, then

$$
\hat{\lambda}_{1} \leq \liminf _{|x| \rightarrow \infty} \frac{p F(z, x)}{|x|^{p}} \leq \limsup _{|x| \rightarrow \infty} \frac{p F(z, x)}{|x|^{p}} \leq \eta_{0} \quad \text { uniformly for a.e. } z \in \Omega
$$

and

$$
\beta_{0} \leq \liminf _{|x| \rightarrow \infty} \frac{p F(z, x)-f(z, x) x}{|x|^{\tau}} \quad \text { uniformly for a.e. } z \in \Omega
$$


(iii): there exist $\delta_{0}>0$ and $\mu \in(1, p)$ such that $\mu F(z, x) \geq f(z, x) x>0$ for a.e. $z \in \Omega$ and for all $0<|x| \leq \delta_{0}$. Moreover, there exist $K>0$ such that $F(z, x) \geq K|x|^{\mu}$ for a.e. $z \in \Omega$ and for all $|x| \leq \delta_{0}$.

\section{Remark 4.1.}

1. It is clear that hypothesis $\boldsymbol{H}_{\mathbf{1}}$ (ii) permits resonance at infinity with respect to the principal eigenvalue $\hat{\lambda}_{1}$; as a consequence, this hypothesis makes the energy functional associated to problem (4.1) (see below) noncoercive.

2. Condition $\boldsymbol{H}_{\mathbf{1}}$ (iii) is a reversed Ambrosetti-Rabinowitz condition near 0 .

3. The second requirement in $\boldsymbol{H}_{\mathbf{1}}$ (iii) is automatically satisfied if $f: \bar{\Omega} \times \mathbb{R} \rightarrow \mathbb{R}$ is continuous, but in general is not true if $f$ is defined only in $\Omega$, unless $\inf _{z \in \Omega} F\left(z, \pm \delta_{0}\right)>0$, although this fact is not noted very often. For example, consider a function $f:(0, \pi) \times \mathbb{R} \rightarrow \mathbb{R}$ which, for $x$ small, is defined as $f(z, x)=$ $\sin z|x|^{\mu-2} x$; then it obviously verifies $\mu F(z, x) \geq f(z, x) x>0$ a.e. in $\Omega$ for all $x \neq 0$, but there is no $K>0$ such that $F(z, x) \geq K|x|^{\mu}$ a.e. in $\Omega$ for $x$ small. See Mugnai [35].

Example 4.2. The following function satisfies assumption $\boldsymbol{H}_{\mathbf{1}}$ (for the sake of simplicity we drop the $z$-dependence):

$$
f(x)= \begin{cases}\theta|x|^{\mu-2} x & \text { if }|x| \leq 1, \\ \eta|x|^{p-2} x-(\eta-\theta)|x|^{q-2} x & \text { if }|x|>1,\end{cases}
$$

with $\theta<\hat{\lambda}_{1} \leq \eta<\hat{\lambda}_{2}$ and $1<q<p, 1<\mu<p$.

Let $\phi: W^{1, p}(\Omega) \rightarrow \mathbb{R}$ be the energy functional associated to problem (4.1), defined by

$$
\phi(u)=\frac{1}{p} \mathscr{E}(u)-\int_{\Omega} F(z, u) d z \quad \text { for all } u \in W^{1, p}(\Omega) .
$$

Evidently, assuming the hypotheses above, $\phi \in C^{1}\left(W^{1, p}(\Omega)\right)$.

Proposition 4.3. If hypotheses $\boldsymbol{H}_{\mathbf{1}}$ (i), (ii) hold and $\beta \in L^{q}(\Omega)$ with $q>\frac{N p}{p-1}$, then $\phi$ satisfies the $C$-condition.

Proof. Let $\left\{u_{n}\right\}_{n \geq 1} \subseteq W^{1, p}(\Omega)$ be a sequence such that

$$
\left|\phi\left(u_{n}\right)\right| \leq M_{2} \quad \text { for some } M_{2}>0 \text { and all } n \geq 1,
$$

and

$$
\left(1+\left\|u_{n}\right\|\right) \phi^{\prime}\left(u_{n}\right) \rightarrow 0 \quad \text { in } W^{1, p}(\Omega)^{*} \text { as } n \rightarrow \infty .
$$

From (4.3) we have

$$
\left|\left\langle\phi^{\prime}\left(u_{n}\right), h\right\rangle\right| \leq \frac{\varepsilon_{n}\|h\|}{1+\left\|u_{n}\right\|} \quad \text { for all } h \in W^{1, p}(\Omega), \text { with } \varepsilon_{n} \rightarrow 0 \text { as } n \rightarrow \infty .
$$


In (4.4) we choose $h=u_{n} \in W^{1, p}(\Omega)$ and obtain

$$
\left|\mathscr{E}\left(u_{n}\right)-\int_{\Omega} f\left(z, u_{n}\right) u_{n} d z\right| \leq \varepsilon_{n} \quad \text { for all } n \geq 1 .
$$

On the other hand, from (4.2) we have

$$
-\mathscr{E}\left(u_{n}\right)+p \int_{\Omega} F\left(z, u_{n}\right) d z \leq p M_{2} \quad \text { for all } n \geq 1 .
$$

We add (4.5) and (4.6) and obtain

$$
\int_{\Omega}\left[p F\left(z, u_{n}\right)-f\left(z, u_{n}\right) u_{n}\right] d z \leq M_{3} \text { for some } M_{3}>0 \text { and all } n \geq 1 \text {. }
$$

By virtue of hypotheses $\boldsymbol{H}_{\mathbf{1}}$ (i), (ii), we can find $\beta_{1} \in\left(0, \beta_{0}\right)$ and $c_{3}>0$ such that

$$
\beta_{1}|x|^{\tau}-c_{3} \leq p F(z, x)-f(z, x) x \quad \text { for a.e. } z \in \Omega \text { and all } x \in \mathbb{R} .
$$

We use (4.7) and (4.8) and obtain that

$$
\left\{u_{n}\right\}_{n \geq 1} \subseteq L^{\tau}(\Omega) \quad \text { is bounded. }
$$

An additional estimate is needed: recall that $\tau \leq p<p^{*}$ (see hypothesis $\boldsymbol{H}_{\mathbf{1}}$ (ii)). So we can find $t \in[0,1)$ such that $\frac{1}{p}=\frac{1-t}{\tau}+\frac{t}{p^{*}}$. Invoking the interpolation inequality, we have

$$
\left\|u_{n}\right\|_{p} \leq\left\|u_{n}\right\|_{\tau}^{1-t}\left\|u_{n}\right\|_{p^{*}}^{t}
$$

and thus there exists $M_{4}>0$ such that

$$
\left\|u_{n}\right\|_{p}^{p} \leq M_{4}\left\|u_{n}\right\|_{p^{*}}^{t p} \quad \text { for all } n \geq 1 .
$$

First suppose that $N \neq p$ and recall that in this case $W^{1, p}(\Omega) \hookrightarrow L^{p^{*}}(\Omega)$ (Sobolev Embedding Theorem, with $p^{*}=\infty$ if $N>p$ ). Thus, from (4.10) we have that there exists $M_{5}>0$ such that

$$
\left\|u_{n}\right\|_{p}^{p} \leq M_{5}\left\|u_{n}\right\|^{t p} \quad \text { for all } n \geq 1 .
$$

Hypothesis $\boldsymbol{H}_{\mathbf{1}}(\mathrm{i})$ implies that

$$
|f(z, x) x| \leq c_{4}\left(1+|x|^{p}\right) \quad \text { for a.e. } z \in \Omega \text {, all } x \in \mathbb{R} \text { and some } c_{4}>0 .
$$

From (4.5) and (4.12) we have

$$
\mathscr{E}\left(u_{n}\right) \leq M_{6}\left(1+\left\|u_{n}\right\|_{p}^{p}\right) \text { for some } M_{6}>0 \text { and all } n \geq 1 ;
$$

and so, by (3.5),

$$
\left\|u_{n}\right\|^{p} \leq M_{7}\left(1+\left\|u_{n}\right\|_{p}^{p}\right) \text { for some } M_{7}>0 \text { and all } n \geq 1 \text {, }
$$


which implies, with (4.11), that

$$
\left\|u_{n}\right\|^{p} \leq M_{8}\left(1+\left\|u_{n}\right\|^{t p}\right) \quad \text { for some } M_{8}>0 \text { and all } n \geq 1 \text {. }
$$

Now recall that $0 \leq t<1$, and so it follows that

$$
\left\{u_{n}\right\}_{n \geq 1} \subseteq W^{1, p}(\Omega) \quad \text { is bounded. }
$$

If $N=p$, then $W^{1, p}(\Omega) \hookrightarrow L^{q}(\Omega)$ for all $q \in[1, \infty)$ by Sobolev Embedding Theorem, and $p^{*}=\infty$. Thus, replacing $p^{*}$ by any $q>p$ in the previous reasoning, we get (4.13) again.

By (4.13) we may assume that

$$
u_{n} \rightarrow u \text { in } W^{1, p}(\Omega) \text { and } u_{n} \rightarrow u \text { in } L^{p q^{\prime}}(\Omega) \text { as } n \rightarrow \infty .
$$

Now in (4.4) we choose $h=u_{n}-u \in W^{1, p}(\Omega)$, we pass to the limit as $n \rightarrow \infty$ and use (4.14) to obtain

$$
\lim _{n \rightarrow \infty}\left\langle A\left(u_{n}\right), u_{n}-u\right\rangle=0
$$

which implies, by Proposition 2.7 , that $u_{n} \rightarrow u$ in $W^{1, p}(\Omega)$, i.e. $\phi$ satisfies the C-condition.

Recall that $\hat{u}_{1} \in C^{1, \alpha}(\Omega)(0<\alpha<1), \hat{u}_{1} \geq 0$ is the $L^{p}$-normalized principal eigenfunction for problem (3.1) (see Proposition 3.1).

Proposition 4.4. If hypotheses $\boldsymbol{H}_{\mathbf{1}}(\mathrm{i})$, (ii) hold and $\beta \in L^{q}(\Omega)$ with $q>\frac{N p}{p-1}$, then $\phi_{\mid \mathbb{R} \hat{u}_{1}}$ is anticoercive, i.e. $\phi\left(t \hat{u}_{1}\right) \rightarrow-\infty$ as $|t| \rightarrow \infty$.

Proof. It is clear from hypothesis $\boldsymbol{H}_{\mathbf{1}}$ (ii) that, without any loss of generality, we may assume that $\tau<p$. Moreover, we can find $\beta_{1} \in\left(0, \beta_{0}\right)$ and $M_{9}=M_{9}\left(\beta_{1}\right)>0$ such that

$$
\beta_{1}|x|^{\tau} \leq p F(z, x)-f(z, x) x \quad \text { for a.e. } z \in \Omega \text { and all } x \geq M_{9} .
$$

Thus (4.15) implies

$$
\frac{d}{d x}\left(\frac{F(z, x)}{x^{p}}\right)=\frac{f(z, x) x-p F(z, x)}{x^{p+1}} \leq-\beta_{1} x^{\tau-p-1}
$$

for a.e. $z \in \Omega$ and all $x \geq M_{9}$.

Integrating (4.16) over $[y, x], M_{9} \leq y \leq x$, we obtain

$$
\frac{F(z, x)}{x^{p}}-\frac{F(z, y)}{y^{p}} \leq \frac{\beta_{1}}{p-\tau}\left(\frac{1}{x^{p-\tau}}-\frac{1}{y^{p-\tau}}\right)
$$

for a.e. $z \in \Omega$ and all $x \geq y \geq M_{9}$. 
We now let $x \rightarrow \infty$; since $\tau<p$, from (4.17) and hypothesis $\boldsymbol{H}_{\mathbf{1}}(\mathrm{ii})$, we have

$$
\frac{\hat{\lambda}_{1}}{p}-\frac{F(z, y)}{y^{p}} \leq-\frac{\beta_{1}}{(p-\tau) y^{p-\tau}} \quad \text { for a.e. } z \in \Omega \text { and all } y \geq M_{9}
$$

that is

$$
\frac{\hat{\lambda}_{1}}{p} y^{p}-F(z, y) \leq-\frac{\beta_{1}}{(p-\tau)} y^{\tau} \quad \text { for a.e. } z \in \Omega \text { and all } y \geq M_{9},
$$

and so

$$
\frac{\hat{\lambda}_{1}}{p} y^{p}-F(z, y) \leq-\frac{\beta_{1}}{(p-\tau)} y^{\tau}+c_{5} \quad \text { for a.e. } z \in \Omega \text { and all } y \geq 0
$$

for some $c_{5}>0$.

Now, take $t>0$, so that by (4.18) we get

$$
\phi\left(t \hat{u}_{1}\right)=\frac{\hat{\lambda}_{1}}{p}\left\|t \hat{u}_{1}\right\|_{p}^{p}-\int_{\Omega} F\left(z, t \hat{u}_{1}\right) d z \leq-\frac{\beta_{1}}{(p-\tau)} t^{\tau}\left\|\hat{u}_{1}\right\|_{\tau}^{\tau}+c_{6}
$$

for some $c_{6}>0$, and so $\phi\left(t \hat{u}_{1}\right) \rightarrow-\infty$ as $t \rightarrow \infty$.

In a similar fashion, we also show that

$$
\phi\left(t \hat{u}_{1}\right) \rightarrow-\infty \quad \text { as } \quad t \rightarrow-\infty
$$

and therefore we can conclude that $\phi_{\mid \mathbb{R} \hat{u}_{1}}$ is anticoercive.

We now introduce the set

$$
S=\left\{u \in W^{1, p}(\Omega): \mathscr{E}(u)=\hat{\lambda}_{2}\|u\|_{p}^{p}\right\}
$$

Proposition 4.5. If hypotheses $\boldsymbol{H}_{1}(\mathrm{i})$, (ii) hold and $\beta \in L^{q}(\Omega)$ with $q>\frac{N p}{p-1}$, then $\phi_{\mid S}$ is coercive.

Proof. By virtue of hypotheses $\boldsymbol{H}_{\mathbf{1}}(\mathrm{i})$, (ii), we can find $\eta_{1} \in\left(\eta_{0}, \hat{\lambda}_{2}\right)$ and $c_{7}>0$ such that

$$
F(z, x) \leq \frac{\eta_{1}}{p}|x|^{p}+c_{7} \quad \text { for a.e. } z \in \Omega \text { and all } x \in \mathbb{R}
$$

Then from (3.5) and (4.19) we have that

$$
\|u\|^{p} \leq c_{8}\|u\|_{p}^{p} \quad \text { for some } c_{8}>0 \text { and all } u \in S .
$$


Therefore, if $u \in S$, we have

$$
\begin{aligned}
\phi(u) & =\frac{1}{p} \mathscr{E}(u)-\int_{\Omega} F(z, u) d z \\
& \geq \frac{1}{p}\left(\hat{\lambda}_{2}-\eta_{1}\right)\|u\|_{p}^{p}-c_{7} \mathscr{L}_{N}(\Omega) \quad(\text { by }(4.20) \text { and the fact that } u \in S) \\
& \geq \frac{1}{c_{8} p}\left(\hat{\lambda}_{2}-\eta_{1}\right)\|u\|^{p}-c_{7} \mathscr{L}_{N}(\Omega) \quad(\text { by }(4.21)),
\end{aligned}
$$

and thus $\phi_{\mid S}$ is coercive, since $\eta_{1}<\hat{\lambda}_{2}$.

Propositions 4.4 and 4.5 imply that we can find $t^{*}>0$ large enough, and bigger than 1 , such that

$$
\phi\left( \pm t^{*} \hat{u}_{1}\right)<\inf _{S} \phi=m_{S} .
$$

Now, set $D_{0}=\left\{ \pm t^{*} \hat{u}_{1}\right\}$ and denote by $D$ the convex hull of $-t^{*} \hat{u}_{1}$ and $t^{*} \hat{u}_{1}$, i.e. $D=\left\{u \in W^{1, p}(\Omega): u=\varepsilon\left(-t^{*} \hat{u}_{1}\right)+(1-\varepsilon)\left(t^{*} \hat{u}_{1}\right), \varepsilon \in[0,1]\right\}$.

Proposition 4.6. If hypotheses $\boldsymbol{H}_{\mathbf{1}}$ (i), (ii) hold and $\beta \in L^{q}(\Omega)$ with $q>\frac{N p}{p-1}$, then the pair $\left\{D_{0}, D\right\}$ is linking with $S$ in $W^{1, p}(\Omega)$ (see Definition 2.1).

Proof. We consider the set $E=\left\{u \in W^{1, p}(\Omega): \mathscr{E}(u)<\hat{\lambda}_{2}\|u\|^{p}\right\}$. Note that $\pm t^{*} \hat{u}_{1} \in E$ by (4.22) and (4.19). We claim that $E$ is not path-connected and that $\pm t^{*} \hat{u}_{1}$ belong to different path components of $E$. To this end, let $\hat{\gamma} \in$ $C\left([-1,1], W^{1, p}(\Omega)\right)$ be a path which joins $-t^{*} \hat{u}_{1}$ and $t^{*} \hat{u}_{1}$. Then

$$
\hat{\gamma}(-1)=-t^{*} \hat{u}_{1} \quad \text { and } \quad \hat{\gamma}(1)=t^{*} \hat{u}_{1} .
$$

We extend $\hat{\gamma}$ along the line segments

$\operatorname{conv}\left\{-t^{*} \hat{u}_{1},-\hat{u}_{1}\right\}=\left\{u \in W^{1, p}(\Omega): u=\varepsilon\left(-t^{*} \hat{u}_{1}\right)+(1-\varepsilon)\left(-\hat{u}_{1}\right), \varepsilon \in[0,1]\right\}$

and

$$
\operatorname{conv}\left\{t^{*} \hat{u}_{1}, \hat{u}_{1}\right\}=\left\{u \in W^{1, p}(\Omega): u=\varepsilon\left(t^{*} \hat{u}_{1}\right)+(1-\varepsilon)\left(\hat{u}_{1}\right), \varepsilon \in[0,1]\right\}
$$

in the obvious linear way, in order to reach $-\hat{u}_{1}$ and $\hat{u}_{1}$ respectively. We denote this extension of $\hat{\gamma}$ by $\hat{\gamma}_{0}$. Note that $\hat{\gamma}_{0} \in \Gamma$ (see Proposition 3.6), and so

$$
\hat{\lambda}_{2} \leq \max _{-1 \leq t \leq 1} \frac{\mathscr{E}\left(\hat{\gamma}_{0}(t)\right)}{\left\|\hat{\gamma}_{0}(t)\right\|_{p}^{p}}
$$

by Proposition 3.6. Thus there exists $t_{0} \in(0,1)$ such that $\hat{\gamma}\left(t_{0}\right) \notin E$ and this shows that $E$ is not path-connected and that the elements $\pm t^{*} \hat{u}_{1}$ belong to different path components of $E$. 
Now take $\gamma \in C\left(D, W^{1, p}(\Omega)\right)$ such that $\gamma_{\mid D_{0}}=I d_{\mid D_{0}}$. Then $\gamma\left(-t^{*} \hat{u}_{1}\right)=-t^{*} \hat{u}_{1}$ and $\gamma\left(t^{*} \hat{u}_{1}\right)=t^{*} \hat{u}_{1}$. Since $\pm t^{*} \hat{u}_{1}$ belong to distinct path components of $E$, we have $\gamma(D) \cap \partial E \neq \emptyset$. But $\partial E \subseteq S$. Hence $\gamma(D) \cap S \neq \emptyset$ and this proves that the pair $\left\{D_{0}, D\right\}$ is linking with $S$ in $W^{1, p}(\Omega)$.

Now we are ready for the first existence result.

Theorem 4.7. If hypotheses $\boldsymbol{H}_{\mathbf{1}}$ hold and $\beta \in L^{q}(\Omega)$ with $q>\frac{N p}{p-1}$, then problem (4.1) has a nontrivial smooth solution $u_{0} \in C^{1, \alpha}(\Omega)(0<\alpha \leq 1)$.

Proof. Propositions 4.3, 4.4, 4.5 and 4.6 permit the use of Theorem 2.2, so we can find $u_{0} \in W^{1, p}(\Omega)$ such that $\phi^{\prime}\left(u_{0}\right)=0$ and, by (4.22),

$$
m_{S} \leq \phi\left(u_{0}\right)=\inf _{\gamma \in \Gamma^{*}} \max _{0 \leq t \leq 1} \phi(\gamma(t)),
$$

where $\Gamma^{*}=\left\{\gamma \in C\left([0,1], W^{1, p}(\Omega)\right): \gamma(0)=-t^{*} \hat{u}_{1}, \gamma(1)=t^{*} \hat{u}_{1}\right\}$. Moreover, we know that the pair $\left\{D_{0}, D\right\}$ and $S$ homologically link in $W^{1, p}(\Omega)$ (see Chang [9, pages 89-90]). Hence, by [9, pages 89-90], we have

$$
C_{1}\left(\phi, u_{0}\right) \neq 0 .
$$

Hypothesis $\boldsymbol{H}_{\mathbf{1}}$ (iii), saying that $F(z, x) \geq K|x|^{\mu}$ for a.e. $z \in \Omega$ and all $|x| \leq \delta_{0}$ for some $K>0$, ensures that we can apply Proposition 2.1 of [24], see also [30], and conclude that

$$
C_{k}(\phi, 0)=0 \quad \text { for all } k \geq 0 .
$$

Comparing (4.23) and (4.24), we see that $u_{0} \neq 0$,and so it is a nontrivial solution of problem (4.1). Nonlinear regularity theory (see Lieberman [29]) implies that $u \in C^{1, \alpha}(\Omega)$ for some $\alpha \in(0,1]$.

If the weight is bounded, we can drop hypothesis $\boldsymbol{H}_{\mathbf{1}}$ (iii), which dictates a $p$-concavity condition near the origin, and replace it by a condition which implies a $p$-linear growth also near the origin, allowing for resonance with respect to the principal eigenvalue $\hat{\lambda}_{1}$ to occur. More precisely, we now consider the following hypotheses for the reaction function $f$ :

$\boldsymbol{H}_{2}: f: \Omega \times \mathbb{R} \rightarrow \mathbb{R}$ is a Carathéodory function such that $f(z, 0)=0$ a.e. in $\Omega$, hypotheses $\boldsymbol{H}_{\mathbf{2}}$ (i), (ii) are the same as the corresponding hypotheses $\boldsymbol{H}_{\mathbf{1}}$ (i), (ii), and

(iii): there exists $\delta_{0}>0$ such that $F(z, x) \leq \frac{\hat{\lambda}_{1}}{p}|x|^{p}$ for a.e. $z \in \Omega$ and all $|x| \leq \delta_{0}$.

Theorem 4.8. If hypotheses $\boldsymbol{H}_{2}$ hold and $\beta \in L^{\infty}(\Omega)$, then problem (4.1) has a nontrivial solution $u_{0} \in C_{n}^{1, \alpha}(\bar{\Omega})(0<\alpha \leq 1)$. 
Proof. Since $\boldsymbol{H}_{\mathbf{1}}$ (i), (ii) hold, we can apply all Proposition 4.3-4.6, so, as before, via Theorem 2.2, we obtain a critical point $u_{0}$ of $\phi$ such that

$$
C_{1}\left(\phi, u_{0}\right) \neq 0 \text {. }
$$

On the other hand, if $\delta_{0}$ is as postulated by hypothesis $\boldsymbol{H}_{\mathbf{2}}$ (iii) and $u \in C_{n}^{1}(\bar{\Omega})$ is such that $\|u\|_{C_{n}^{1}(\bar{\Omega})}<\delta_{0}$, then $F(z, u(z)) \leq \frac{\hat{\lambda}_{1}}{p} u(z)^{p}$ a.e. in $\Omega$, and so, by (3.2),

$$
\phi(u) \geq \frac{1}{p} \mathscr{E}(u)-\frac{\hat{\lambda}_{1}}{p}\|u\|_{p}^{p} \geq 0=\phi(0)
$$

for all $u \in C_{n}^{1}(\bar{\Omega})$ with $\|u\|_{C_{n}^{1}(\bar{\Omega})} \leq \delta_{0}$. Thus $u=0$ is a local $C_{n}^{1}(\bar{\Omega})$-minimizer of $\phi$. Hence, by Theorem $2.5, u=0$ is also a local $W_{n}^{1, p}(\Omega)$-minimizer for $\phi$. By (2.2) we have that

$$
C_{k}(\phi, 0)=\delta_{k, 0} \mathbb{Z} \quad \text { for all } k \geq 0 .
$$

Comparing (4.25) and (4.26), we conclude that $u_{0} \neq 0$. Moreover, $u_{0} \in C_{n}^{1, \alpha}(\bar{\Omega})$ $(0<\alpha \leq 1)$, see Lieberman [29].

We have another existence result for resonant Neumann problems with the following assumptions on $f$ :

$\boldsymbol{H}_{\mathbf{3}}: f: \Omega \times \mathbb{R} \rightarrow \mathbb{R}$ is a Carathéodory function such that $f(z, 0)=0$ a.e. in $\Omega$, hypothesis $\boldsymbol{H}_{\mathbf{3}}(\mathrm{i})$ is the same as the corresponding hypothesis $\boldsymbol{H}_{\mathbf{1}}(\mathrm{i})$,

(ii): $\lim _{|x| \rightarrow \infty} \frac{p F(z, x)}{|x|^{p}}=\hat{\lambda}_{1}$ uniformly for a.e. $z \in \Omega$ and

$$
\lim _{|x| \rightarrow \infty}[p F(z, x)-f(z, x) x]=-\infty \quad \text { uniformly for a.e. } z \in \Omega
$$

(iii): there exists $\theta \in L^{\infty}(\Omega)$ such that

$$
\theta(z) \leq \hat{\lambda}_{1} \quad \text { a.e. in } \Omega, \quad \theta \neq \hat{\lambda}_{1}
$$

and

$$
\lim _{x \rightarrow 0} \frac{f(z, x)}{|x|^{p-2} x} \leq \theta(z) \quad \text { uniformly for a.e. } z \in \Omega .
$$

Remark 4.9. Hypothesis $\boldsymbol{H}_{3}$ (ii) implies that the problem is resonant at infinity with respect to the principal eigenvalue $\hat{\lambda}_{1}$ from the left.

Example 4.10. The following function satisfies hypotheses $\boldsymbol{H}_{\mathbf{3}}$ (again we drop the $z$-dependence):

$$
f(x)= \begin{cases}\theta|x|^{p-2} x & \text { if }|x| \leq 1, \\ \hat{\lambda}_{1}|x|^{p-2} x-\hat{c} \frac{x}{|x|^{2}} & \text { if }|x|>1,\end{cases}
$$

where $\theta<\hat{\lambda}_{1}, \hat{c}=\hat{\lambda}_{1}-\theta>0$ and $1 \leq r<\infty$. Note that such a function does not satisfy $\boldsymbol{H}_{\mathbf{1}}$ (ii).

We start with a simple lemma which will be useful also in the next section. 
Lemma 4.11. If $\theta \in L^{\infty}(\Omega), \theta \leq \hat{\lambda}_{1}$ a.e. in $\Omega$ and $\theta \neq \hat{\lambda}_{1}$, then there exists $\mu_{0}>0$ such that

$$
\psi(u)=\mathscr{E}(u)-\int_{\Omega} \theta|u|^{p} d z \geq \mu_{0}\|u\|^{p} \quad \text { for all } u \in W^{1, p}(\Omega) .
$$

Proof. Evidently $\psi \geq 0$. Suppose that the lemma is not true. Then, exploiting the $p$-homogeneity of $\psi$, we can find a sequence $\left\{u_{n}\right\}_{n \geq 1} \subseteq W^{1, p}(\Omega)$ such that

$$
\left\|u_{n}\right\|=1 \quad \text { for all } n \geq 1 \quad \text { and } \psi\left(u_{n}\right) \rightarrow 0^{+} \quad \text { as } n \rightarrow \infty .
$$

We may assume that

$$
u_{n} \rightarrow u \quad \text { in } W^{1, p}(\Omega) \quad \text { and } u_{n} \rightarrow u \quad \text { in } L^{p}(\Omega) \quad \text { as } n \rightarrow \infty .
$$

It follows from (4.27) and the lower weak semicontinuity of $\mathscr{E}$ that $\psi(u) \leq 0$, and so

$$
\mathscr{E}(u)=\int_{\Omega} \theta|u|^{p} d z \leq \hat{\lambda}_{1}\|u\|_{p}^{p} .
$$

If $u=0$, then from (3.5) applied to $u_{n}$ and (4.27), we see that $u_{n} \rightarrow 0$ in $W^{1, p}(\Omega)$, a contradiction to the fact that $\left\|u_{n}\right\|=1$ for all $n \geq 1$. Hence $u \neq 0$. But from (4.28) and (3.2) we have $\mathscr{E}(u)=\hat{\lambda}_{1}\|u\|_{p}^{p}$, and so $u= \pm t \hat{u}_{1}$ for some $t>0$. Recalling that $\hat{u}_{1}(z)>0$ for all $z \in \Omega$ (see Proposition 3.1), from (4.28) and the hypothesis on $\theta$, we have $\mathscr{E}(u)<\hat{\lambda}_{1}\|u\|_{p}^{p}$, again a contradiction. The lemma is thus proved.

Proposition 4.12. If hypotheses $\boldsymbol{H}_{3}(\mathrm{i})$, (ii) hold, and $\beta \in L^{q}(\Omega)$ with $q>\frac{N p}{p-1}$, then $\phi$ satisfies the $C$-condition.

Proof. Let $\left\{u_{n}\right\}_{n \geq 1} \subseteq W^{1, p}(\Omega)$ be a C-sequence satisfying (4.2) and (4.3). Then, as in the proof of Proposition 4.3, starting from (4.2) and (4.3) but using the reversed signs, we obtain an inequality opposite to (4.7), i.e.

$$
\int_{\Omega}\left[p F\left(z, u_{n}\right)-f\left(z, u_{n}\right) u_{n}\right] d z \geq M_{10} \text { for some } M_{10}>0 \text { and all } n \geq 1 \text {. }
$$

Claim: $\left\{u_{n}\right\}_{n \geq 1} \subseteq W^{1, p}(\Omega)$ is bounded.

Suppose that the Claim is not true. Then, by passing to a suitable subsequence if necessary, we may assume that $\left\|u_{n}\right\| \rightarrow \infty$. Let $y_{n}=u_{n} /\left\|u_{n}\right\|, n \geq 1$. Then $\left\|y_{n}\right\|=1$ for all $n \geq 1$ and so we may assume that

$$
y_{n} \rightarrow y \quad \text { in } W^{1, p}(\Omega) \quad \text { and } y_{n} \rightarrow y \quad \text { in } L^{p q^{\prime}}(\Omega) \quad \text { as } n \rightarrow \infty .
$$

Let $F(z, x)=\frac{\hat{\lambda}_{1}}{p}|x|^{p}+F_{0}(z, x)$. It is clear from hypothesis $\boldsymbol{H}_{\mathbf{3}}$ (ii) that

$$
\lim _{|x| \rightarrow \infty} \frac{F_{0}(z, x)}{|x|^{p}}=0 \quad \text { uniformly for a.e. } z \in \Omega \text {. }
$$


Then (4.31), and hypothesis $\boldsymbol{H}_{\mathbf{3}}(\mathrm{i})$ imply that, given $\varepsilon>0$, there exists $C_{\varepsilon}>0$ such that

$$
F_{0}(z, x) \leq \frac{\varepsilon}{p}|x|^{p}+c_{\varepsilon} \quad \text { for a.e. } z \in \Omega \text { and all } x \in \mathbb{R} .
$$

From (4.2), recalling that $\left\{u_{n}\right\}_{n \geq 1}$ is a C-sequence, we have that for all $n \geq 1$

$$
\begin{aligned}
M_{2} \geq \phi\left(u_{n}\right) & =\frac{1}{p} \mathscr{E}\left(u_{n}\right)-\frac{\hat{\lambda}_{1}}{p}\left\|u_{n}\right\|_{p}^{p}-\int_{\Omega} F_{0}\left(z, u_{n}\right) d z \\
& \geq \frac{1}{p} \mathscr{E}\left(u_{n}\right)-\frac{\hat{\lambda}_{1}+\varepsilon}{p}\left\|u_{n}\right\|_{p}^{p}-c_{\varepsilon} \mathscr{L}_{N}(\Omega),
\end{aligned}
$$

and hence

$$
\frac{M_{2}}{\left\|u_{n}\right\|^{p}} \geq \frac{1}{p} \mathscr{E}\left(y_{n}\right)-\frac{\hat{\lambda}_{1}+\varepsilon}{p}\left\|y_{n}\right\|_{p}^{p}-\frac{c_{\varepsilon} \mathscr{L}_{N}(\Omega)}{\left\|u_{n}\right\|^{p}},
$$

and so, by (4.30),

$$
\mathscr{E}(y) \leq\left(\hat{\lambda}_{1}+\varepsilon\right)\|y\|_{p}^{p}
$$

Since $\varepsilon>0$ was arbitrary, we let $\varepsilon \rightarrow 0^{+}$and obtain

$$
\mathscr{E}(y) \leq \hat{\lambda}_{1}\|y\|_{p}^{p},
$$

and by Proposition 3.1

$$
\mathscr{E}(y)=\hat{\lambda}_{1}\|y\|_{p}^{p} .
$$

Thus, either $y=0$ or $y= \pm t \hat{u}_{1}$ for some $t>0$.

If $y=0$, then $y_{n} \rightarrow 0$ in $W^{1, p}(\Omega)$ by (3.5), a contradiction to the fact that $\left\|y_{n}\right\|=1$ for all $n \geq 1$. So $y= \pm t \hat{u}_{1}$, and since $\hat{u}_{1}(z)>0$ for all $z \in \Omega$, it follows that $\left|u_{n}(z)\right| \rightarrow \infty$ for all $z \in \Omega$ as $n \rightarrow \infty$. Thus, by $\boldsymbol{H}_{3}$ (ii) we have

$$
\lim _{n \rightarrow \infty}\left[p F\left(z, u_{n}\right)-f\left(z, u_{n}\right) u_{n}\right]=-\infty \quad \text { for all } z \in \Omega \text { as } n \rightarrow \infty,
$$

and by Fatou's Lemma we get

$$
\lim _{n \rightarrow \infty} \int_{\Omega}\left[p F\left(z, u_{n}\right)-f\left(z, u_{n}\right) u_{n}\right] d z=-\infty,
$$

which contradicts (4.29). This proves the claim.

Because of the Claim, we may assume that

$$
u_{n} \rightarrow u \quad \text { in } W^{1, p}(\Omega) \quad \text { and } u_{n} \rightarrow u \quad \text { in } L^{p q^{\prime}}(\Omega) \quad \text { as } n \rightarrow \infty .
$$

From this, using Proposition 2.7 and acting as in the proof of Proposition 4.3, we conclude that $\phi$ satisfies the C-condition. 
Proposition 4.13. If $\boldsymbol{H}_{3}(\mathrm{i})$, (ii) hold and $\beta \in L^{q}(\Omega)$ with $q>\frac{p N}{p-1}$, then $\phi_{\mid \mathbb{R} \hat{u}_{1}}$ is anticoercive.

Proof. The proof is similar to that of Proposition 4.4, and so we only sketch it. By $\boldsymbol{H}_{\mathbf{3}}$ (ii) it is readily seen that also

$$
\lim _{|x| \rightarrow \infty}\left[p F_{0}(z, x)-f_{0}(z, x) x\right]=-\infty \quad \text { uniformly for a.e. } z \in \Omega .
$$

Then

$$
\frac{d}{d x}\left(\frac{F_{0}(z, x)}{x^{p}}\right)=\frac{f_{0}(z, x) x-p F_{0}(z, x)}{x^{p+1}} \geq 0 \quad \text { for a.e. } z \in \Omega \text { and all }|x| \geq M,
$$

for some $M>0$. Integrating over $[M, x]$, we get $F_{0}(z, x) \geq x^{p} F_{0}(z, M) / M^{p}$ and analogously for $x<0$. Hence $\lim _{|x| \rightarrow \infty} F_{0}(z, x)=\infty$ uniformly for a.e. $z \in \Omega$. Thus

$$
\lim _{|t| \rightarrow \infty} \phi\left(t \hat{u}_{1}\right)=-\lim _{|t| \rightarrow \infty} \int_{\Omega} F_{0}\left(z, t \hat{u}_{1}\right) d z=-\infty
$$

and the proof is complete.

Using hypothesis $\boldsymbol{H}_{\mathbf{3}}$ (iii) and Lemma 4.11 we can now show the following proposition.

Proposition 4.14. If $\boldsymbol{H}_{\mathbf{3}}(\mathrm{i})$, (iii) hold and $\beta \in L^{q}(\Omega)$ with $q>\frac{N p}{p-1}$, then $u=0$ is a local minimizer of $\phi$.

Proof. By virtue of hypotheses $\boldsymbol{H}_{\mathbf{3}}$ (i), (iii), given any $\varepsilon>0$, we can find $c_{\varepsilon}>0$ such that

$$
F(z, x) \leq \frac{1}{p}(\theta(z)+\varepsilon)|x|^{p}+c_{\varepsilon}|x|^{r} \quad \text { for a.e. } z \in \Omega \text {, all } x \in \mathbb{R} \text {, with } r>p \text {. }
$$

Then for every $u \in W^{1, p}(\Omega)$ we have

$$
\begin{aligned}
\phi(u) & =\frac{1}{p} \mathscr{E}(u)-\int_{\Omega} F(z, u) d z \\
& \geq \frac{1}{p} \mathscr{E}(u)-\frac{1}{p} \int_{\Omega} \theta|u|^{p} d z-\frac{\varepsilon}{p}\|u\|^{p}-c_{9}\|u\|^{r} \quad \text { by (4.33) } \\
& \geq \frac{\mu_{0}-\varepsilon}{p}\|u\|^{p}-c_{9}\|u\|^{r} \quad \text { by Lemma 4.11. }
\end{aligned}
$$

Choosing $\varepsilon \in\left(0, \mu_{0}\right)$, from (4.34) we have

$$
\phi(u) \geq c_{10}\|u\|^{p}-c_{9}\|u\|^{r} \quad \text { for some } c_{10}>0 .
$$

Since $r>p$, from (4.35) we see that 0 is a local minimizer for $\phi$. 
Now we are ready for the new existence theorem.

Theorem 4.15. If hypotheses $\boldsymbol{H}_{3}$ hold and $\beta \in L^{q}(\Omega)$ with $q>\frac{N p}{p-1}$, then problem (4.1) has a nontrivial solution $u_{0} \in C^{1, \alpha}(\Omega)(0<\alpha \leq 1)$.

Proof. From Proposition 4.14 we know that $u=0$ is a local minimizer of $\phi$. By (4.35) we actually have that $u=0$ is a strict local minimizer. Hence we can find $\rho>0$ small enough such that

$$
\phi(0)=0<\inf \{\phi(u):\|u\|=\rho\}=\eta_{\rho} .
$$

Then Propositions 4.12 and 4.13 and (4.36) above allow us to use Theorem 2.3 (the Mountain Pass Theorem), and so we obtain $u_{0} \in W^{1, p}(\Omega)$ such that

$$
\phi^{\prime}\left(u_{0}\right)=0 \quad \text { and } \quad \phi(0)=0<\eta_{\rho} \leq \phi\left(u_{0}\right) .
$$

From the inequality in (4.37) we see that $u_{0} \neq 0$; from the equality we have that $u_{0}$ solves (4.1). Moreover, $u_{0} \in C^{1, \alpha}(\Omega)(0<\alpha \leq 1)$ by nonlinear regularity theory.

As before, we can modify the behaviour of $f(z, \cdot)$ near 0 , assume that the weight is bounded and produce a new existence theorem. So the new hypotheses on $f$ are the following:

$\boldsymbol{H}_{\mathbf{4}}: f: \Omega \times \mathbb{R} \rightarrow \mathbb{R}$ is a Carthéodory function such that $f(z, 0)=0$ a.e. in $\Omega$, hypotheses $\boldsymbol{H}_{\mathbf{4}}$ (i), (ii) are the same as the corresponding hypotheses $\boldsymbol{H}_{\mathbf{3}}$ (i), (ii) and

(iii): there exist $\hat{\beta}>\hat{\lambda}_{2}$ and $\delta_{0}>0$ such that

$$
\frac{\hat{\beta}}{p}|x|^{p} \leq F(z, x) \quad \text { for a.e. } z \in \Omega \text { and all }|x| \leq \delta_{0}
$$

and

$$
\hat{\lambda}_{1}|x|^{p} \leq f(z, x) x \quad \text { for a.e. } z \in \Omega \text { and all } x \in \mathbb{R} .
$$

Remark 4.16. Hypothesis $\boldsymbol{H}_{\mathbf{4}}$ (iii) implies that $u=0$ is not a local minimizer of $\phi$ and so the mountain pass geometry fails. Thus our approach will be based on Theorem 2.2. Note that Propositions 4.12 and 4.13 still remain valid under this new set of assumptions.

Theorem 4.17. If hypotheses $\boldsymbol{H}_{\mathbf{4}}$ hold and $\beta \in L^{\infty}(\Omega)$, then problem (4.1) has a nontrivial solution $u_{0} \in C^{1, \alpha}(\bar{\Omega})(0<\alpha \leq 1)$. 
Proof. As before, we write $F(z, x)=\frac{\hat{\lambda}_{1}}{p}|x|^{p}+F_{0}(z, x)$. Then for all $u \in V$ (see (3.20)), we have

$$
\begin{aligned}
\phi(u) & =\frac{1}{p} \mathscr{E}(u)-\frac{\hat{\lambda}_{1}}{p}\|u\|_{p}^{p}-\int_{\Omega} F_{0}(z, u) d z \\
& \left.\geq \frac{1}{p} \mathscr{E}(u)-\frac{\hat{\lambda}_{1}}{p \hat{\lambda}_{V}} \mathscr{E}(u)-\frac{\varepsilon}{p}\|u\|_{p}^{p}-c_{\varepsilon} \mathscr{L}_{N}(\Omega) \quad \text { see }(3.20) \text { and }(4.32)\right) \\
& \geq \frac{1}{p}\left(1-\frac{\hat{\lambda}_{1}+\varepsilon}{\hat{\lambda}_{V}}\right) \mathscr{E}(u)-c_{\varepsilon} \mathscr{L}_{N}(\Omega) \quad \text { by }(3.20) .
\end{aligned}
$$

Now choose $\varepsilon \in\left(\hat{\lambda}_{1}, \hat{\lambda}_{V}\right)$ (see Proposition 3.8). Then, from (4.38) we see that $\phi_{\mid V}$ is coercive, and so it is bounded below. Using Proposition 4.13, we can find $t^{*}>0$ large enough such that $\phi\left( \pm t^{*} \hat{u}_{1}\right)<m_{V}=\inf _{V} \phi$.

We consider the sets $D_{0}=\left\{ \pm t^{*} \hat{u}_{1}\right\}$, the convex hull

$D=\left[-t^{*} \hat{u}_{1}, t^{*} \hat{u}_{1}\right]=\left\{u \in W^{1, p}(\Omega): u=\varepsilon\left(-t^{*} \hat{u}_{1}\right)+(1-\varepsilon) t^{*} \hat{u}_{1}, 0 \leq \varepsilon \leq 1\right\}$

and the already introduced set $V=\left\{u \in W^{1, p}(\Omega): \int_{\Omega} \hat{u}_{1} u d z=0\right\}$. As in Proposition 4.6, we check that the pair $\left\{D_{0}, D\right\}$ is linking with $V$ in $W^{1, p}(\Omega)$. So we can apply Theorem 2.2 and obtain $u_{0} \in W^{1, p}(\Omega)$ such that

$$
\phi^{\prime}\left(u_{0}\right)=0 \quad \text { and } \quad m_{V} \leq \phi\left(u_{0}\right)=c_{0}=\inf _{\hat{\gamma} \in \hat{\Gamma}} \max _{t \in[0,1]} \phi(\hat{\gamma}(t)),
$$

where

$$
\hat{\Gamma}=\left\{\hat{\gamma} \in C\left([0,1], W^{1, p}(\Omega): \hat{\gamma}(0)=t^{*} \hat{u}_{1}, \hat{\gamma}(1)=-t^{*} \hat{u}_{1}\right\} .\right.
$$

From the first equation in (4.39) we see that $u_{0}$ is a solution of (4.1) and $u_{0} \in$ $C^{1, \alpha}(\bar{\Omega})(0<\alpha \leq 1)$, see Lieberman [29].

We need to show the nontriviality of $u_{0}$. According to the minimax expression of $\phi\left(u_{0}\right)=c_{0}$ in (4.39), it suffices to produce a path $\hat{\gamma}_{*} \in \hat{\Gamma}$ such that $\phi_{\mid \hat{\gamma}_{*}}<0$.

To this end, let $M_{C}=M \cap C^{1}(\bar{\Omega})$. We consider $M$ endowed with the relative $W^{1, p}(\Omega)$-topology and $M_{C}$ furnished with the relative $C^{1}(\bar{\Omega})$-topology. Evidently $M_{C}$ is dense in $M$. Now, let

$$
\Gamma=\left\{\gamma \in C([-1,1], M): \gamma(-1)=-\hat{u}_{1}, \gamma(1)=\hat{u}_{1}\right\}
$$

and

$$
\Gamma_{C}=\left\{\gamma \in C\left([-1,1], M_{C}\right): \gamma(-1)=-\hat{u}_{1}, \gamma(1)=\hat{u}_{1}\right\} .
$$


Then it is clear that $\Gamma_{C}$ is dense in $\Gamma$, and so, invoking Proposition 3.6, we see that, given $\delta>0$, we can find $\tilde{\gamma}=\tilde{\gamma}_{\delta} \in \Gamma_{C}$ such that

$$
\max \{\mathscr{E}(\tilde{\gamma}(t)):-1 \leq t \leq 1\} \leq \hat{\lambda}_{2}+\delta .
$$

Since $\tilde{\gamma} \in \Gamma_{C}$, we can find $\mu>0$ small enough such that $\mu|\tilde{\gamma}(t)(z)| \leq \delta_{0}$ for all $t \in[-1,1]$ and all $z \in \bar{\Omega}$, with $\delta_{0}$ as postulated by hypothesis $\boldsymbol{H}_{\mathbf{4}}$ (iii). Then

$$
F(z, \mu \tilde{\gamma}(t)(z)) \geq \frac{\hat{\beta}}{p}|\mu \tilde{\gamma}(t)(z)|^{p} \quad \text { for all } t \in[-1,1] \text { and a.e. } z \in \Omega \text {. }
$$

Therefore, for every $t \in[-1,1]$, we have

$$
\begin{aligned}
\phi(\mu \tilde{\gamma}(t)) & =\frac{\mu^{p}}{p} \mathscr{E}(\tilde{\gamma}(t))-\int_{\Omega} F(z, \mu \tilde{\gamma}(t)) d z \\
& \leq \frac{\mu^{p}}{p}\left(\hat{\lambda}_{2}+\delta\right)-\frac{\hat{\beta}}{p} \mu^{p}\|\tilde{\gamma}(t)\|_{p}^{p} \quad \text { by (4.40) and (4.41) } \\
& =\frac{\mu^{p}}{p}\left(\hat{\lambda}_{2}+\delta-\hat{\beta}\right),
\end{aligned}
$$

since $\tilde{\gamma}$ has values in $M$.

Now recall that $\hat{\beta}>\hat{\lambda}_{2}$ (see $\boldsymbol{H}_{\mathbf{4}}$ (iii)). So, choosing $\delta \in\left(0, \hat{\beta}-\hat{\lambda}_{2}\right)$, we infer that $\phi(\mu \tilde{\gamma}(t))<0$ for all $t \in[-1,1]$, and so

$$
\phi_{\mid \mu \tilde{\gamma}}<0 .
$$

Of course, we can always assume $\mu<t^{*}$, and so we can consider $\sigma:\left[\mu, t^{*}\right] \rightarrow \mathbb{R}$ defined by $\sigma(s)=\phi\left(s \hat{u}_{1}\right)$. Then $\sigma$ is of class $C^{1}$ and

$$
\sigma^{\prime}(s)=\left\langle\phi^{\prime}\left(s \hat{u}_{1}\right), \hat{u}_{1}\right\rangle=\frac{1}{s}\left(\mathscr{E}\left(s \hat{u}_{1}\right)-\int_{\Omega} f\left(z, s \hat{u}_{1}\right) \hat{u}_{1}\right) \leq 0
$$

by $\boldsymbol{H}_{\mathbf{4}}(\mathrm{iii})$, and so

$$
\sigma(s) \leq \sigma(\mu) \quad \text { for all } s \in\left[\mu, t^{*}\right] .
$$

Now, we consider the linear path $\gamma_{+}$joining $t^{*} \hat{u}_{1}$ and $\mu \hat{u}_{1}$. Then from (4.43) and (4.44), we see that

$$
\phi_{\mid \gamma_{+}}<0
$$

We continue with $\tilde{\gamma}_{\mu}=\mu \tilde{\gamma}$, which joins $\mu \hat{u}_{1}$ and $-\mu \hat{u}_{1}$, and along it we have (4.43). Finally, reasoning as in the construction of $\gamma_{+}$, we produce a path $\gamma_{-}$joining $-\mu \hat{u}_{1}$ and $-t \hat{u}_{1}$ such that

$$
\phi_{\mid \gamma_{-}}<0 \text {. }
$$

We now concatenate $\gamma_{+}, \tilde{\gamma}_{\mu}$ and $\gamma_{-}$, producing a path $\hat{\gamma}_{*} \in \hat{\Gamma}$ such that, due to (4.43), (4.45) and (4.46),

$$
\phi_{\mid \hat{\gamma}_{*}}<0,
$$

and so $u_{0} \neq 0$. 


\section{Nonlinear problems: multiplicity theorems}

In this section we prove two multiplicity results for problem (4.1). In the first one we assume that the weight function $\beta$ is bounded, while in the second case we deal with an unbounded weight function $\beta$.

For the first multiplicity result the hypotheses on $f$ are the following:

H5 $_{\mathbf{5}} f: \Omega \times \mathbb{R} \rightarrow \mathbb{R}$ is a Carathéodory function such that $f(z, 0)=0$ a.e. in $\Omega$, hypotheses $\boldsymbol{H}_{\mathbf{5}}(\mathrm{i})$, (iii) are the same as the corresponding hypotheses $\boldsymbol{H}_{\mathbf{1}}$ (i), (iii) and

(ii): there exist two functions $w_{ \pm} \in W^{1, p}(\Omega) \cap C(\bar{\Omega})$ such that

$$
\begin{gathered}
w_{-}(z) \leq c_{-}<0<c_{+} \leq w_{+} \quad \text { for all } z \in \bar{\Omega} \\
\underset{\Omega}{\operatorname{ess} \sup }\left\{f\left(\cdot, w_{+}(\cdot)\right)-\beta(\cdot) w_{+}(\cdot)^{p-1}\right\}<0 \\
<\underset{\Omega}{\operatorname{essinf}}\left\{f\left(\cdot, w_{-}(\cdot)\right)-\beta(\cdot)\left|w_{-}(\cdot)\right|^{p-2} w_{-}(\cdot)\right\}, \\
A\left(w_{-}\right) \leq 0 \leq A\left(w_{+}\right) \quad \text { in } W^{1, p}(\Omega)^{*}
\end{gathered}
$$

and for $r=\max \left\{\left\|w_{-}\right\|_{\infty},\left\|w_{+}\right\|_{\infty}\right\}$, there exists $\xi_{r}>0$ such that for a.e. $z \in \Omega$ the function

$$
x \mapsto f(z, x)+\xi_{r}|x|^{p-2} x
$$

is nondecreasing on $[-r, r]$.

Remark 5.1. Since $\boldsymbol{H}_{5}$ includes part of hypotheses $\boldsymbol{H}_{\mathbf{1}}$, it is clear that again we permit resonance with respect to the principal eigenvalue $\hat{\lambda}_{1}$.

Example 5.2. The following function satisfies hypotheses $\boldsymbol{H}_{\mathbf{5}}$ (again we have dropped the $z$-dependence):

$$
f(x)= \begin{cases}|x|^{q-2} x-\xi|x|^{p-2} x & \text { if }|x| \leq 1 \\ \eta|x|^{p-2} x-\theta|x|^{\tau-2} x & \text { if }|x|>1\end{cases}
$$

with $1<q, \tau<p, \xi>1+\|\beta\|_{\infty}, \eta \in\left[\hat{\lambda}_{1}, \hat{\lambda}_{2}\right)$ and $\theta=\eta+\xi-1$.

Example 5.3. With the function defined in the example above, a natural choice is to take $w_{-}=-1$ and $w_{+}=1$.

First we produce constant sign smooth (i.e. $\left.C^{1, \alpha}(\bar{\Omega})\right)$ solutions.

Proposition 5.4. If hypotheses $\boldsymbol{H}_{5}$ hold and $\beta \in L^{\infty}(\Omega)$, then problem (4.1) has two nontrival constant sign smooth solutions

$$
\begin{aligned}
& u_{0} \in \operatorname{int} C_{+} \text {satisfying } w_{+}-u_{0} \in \operatorname{int} C_{+} \\
& v_{0} \in-\operatorname{int} C_{+} \text {satisfying } v_{0}-w_{-} \in \operatorname{int} C_{+}
\end{aligned}
$$

and both solutions are local minimizers of the energy functional $\phi$. 
Proof. First we show the existence of the positive smooth solution. To this end, take $\lambda>\|\beta\|_{\infty}$ and consider the following truncation-perturbation of the reaction term $f$ :

$$
g_{\lambda}^{+}(z, x)= \begin{cases}0 & \text { if } x \leq 0, \\ f(z, x)+\lambda x^{p-1} & \text { if } 0 \leq x \leq w_{+}(z), \\ f\left(z, w_{+}(z)\right)+\lambda w_{+}(z)^{p-1} & \text { if } w_{+}(z) \leq x .\end{cases}
$$

Of course, this is a Carathéodory function. We set $G_{\lambda}^{+}(z, x)=\int_{0}^{x} g_{\lambda}^{+}(z, s) d s$ and we introduce the $C^{1}$ functional $\phi_{\lambda}^{+}: W_{n}^{1, p}(\Omega) \rightarrow \mathbb{R}$ defined by

$$
\phi_{\lambda}^{+}(u)=\frac{1}{p} \mathscr{E}(u)+\frac{\lambda}{p}\|u\|_{p}^{p}-\int_{\Omega} G_{\lambda}^{+}(z, u) d z \quad \text { for all } u \in W_{n}^{1, p}(\Omega) .
$$

From (5.1) and since $\lambda>\|\beta\|_{\infty}$, we see that $\phi_{\lambda}^{+}$is coercive. Moreover, exploiting the compact embedding of $W_{n}^{1, p}(\Omega)$ into $L^{p q^{\prime}}(\Omega)$, we easily check that $\phi_{\lambda}^{+}$is sequentially weakly lower semicontinuous. Thus, by the Weierstrass Theorem, there exists $u_{0} \in W_{n}^{1, p}(\Omega)$ such that

$$
\phi_{\lambda}^{+}\left(u_{0}\right)=\inf \left\{\phi_{\lambda}^{+}(u): u \in W_{n}^{1, p}(\Omega)\right\}=m_{\lambda}^{+} .
$$

Of course, we can always assume that $\delta_{0}<\min \left\{1, c_{+}\right\}$, see hypotheses $\boldsymbol{H}_{\mathbf{5}}$ (ii), (iii). Moreover, recall that by $\boldsymbol{H}_{\mathbf{5}}$ (iii) there exist $\delta_{0}>0, \mu \in(1, p)$ and $K>0$ such that

$$
F(z, x) \geq K|x|^{\mu} \text { a.e. in } \Omega \text { for all }|x| \leq \delta_{0} .
$$

Then for any $\varepsilon \in\left(0, \delta_{0}\right]$ we have (recall that constant functions belong to $W_{n}^{1, p}(\Omega)$ )

$$
\begin{aligned}
\phi_{\lambda}^{+}(\varepsilon) & \leq \frac{\|\beta\|_{\infty}}{p} \varepsilon^{p}-\int_{\Omega} F(z, \varepsilon) d z \quad \text { see (5.1) } \\
& \leq \frac{\|\beta\|_{\infty}}{p} \varepsilon^{p}-K \varepsilon^{\mu} \mathscr{L}_{N}(\Omega) .
\end{aligned}
$$

Since $\mu<p$, from (5.3) we see that by choosing $\varepsilon \in\left(0, \delta_{0}\right]$, even smaller if necessary, we will have $\phi_{\lambda}^{+}(\varepsilon)<0$. Thus

$$
m_{\lambda}^{+}=\phi_{\lambda}^{+}\left(u_{0}\right)<0=\phi_{\lambda}^{+}(0),
$$

and so $u_{0} \neq 0$.

Now, recall that $\left(\phi_{\lambda}^{+}\right)^{\prime}\left(u_{0}\right)=0$, that is

$$
A\left(u_{0}\right)+(\lambda+\beta)\left|u_{0}\right|^{p-2} u_{0}=N_{\lambda}^{+}\left(u_{0}\right),
$$

where

$$
N_{\lambda}^{+}(u)(\cdot)=g_{\lambda}^{+}(\cdot, u(\cdot)) \quad \text { for all } u \in W_{n}^{1, p}(\Omega) .
$$


On (5.4) we act with $-u_{0}^{-} \in W_{n}^{1, p}(\Omega)$ and obtain

$$
-\int_{\Omega}\left|D u_{0}^{-}\right|_{N}^{p} d z-\int_{\Omega}(\beta+\lambda)\left(u_{0}^{-}\right)^{p} d z=0 \quad \text { see }(5.1),
$$

and so $\left\|u_{0}^{-}\right\|=0$, i.e.

$$
u_{0} \geq 0, u_{0} \neq 0 \text {. }
$$

Then

$$
A\left(u_{0}\right)+(\lambda+\beta) u_{0}^{p-1}=N_{\lambda}^{+}\left(u_{0}\right),
$$

that is

$$
\begin{cases}-\Delta_{p} u_{0}+(\lambda+\beta) u_{0}^{p-1}=g_{\lambda}^{+}\left(z, u_{0}\right) & \text { a.e. in } \Omega \\ \frac{\partial u}{\partial n}=0 & \text { on } \partial \Omega .\end{cases}
$$

By nonlinear regularity theory (see [23] and [29]), we have that $u_{0} \in C_{+} \backslash\{0\}$. obtain

Next we act on (5.4) with $\left(u_{0}-w_{+}\right)^{+} \in W_{n}^{1, p}(\Omega)$, and recalling (5.1), we

$$
\begin{aligned}
\left\langle A\left(u_{0}\right),\left(u_{0}-w_{+}\right)^{+}\right\rangle & +\int_{\Omega}(\lambda+\beta) u_{0}^{p-1}\left(u_{0}-w_{+}\right)^{+} d z \\
& =\int_{\Omega}\left(f\left(z, w_{+}\right)+\lambda w_{+}^{p-1}\right)\left(u_{0}-w_{+}\right)^{+} d z \\
& \leq \int_{\Omega}(\lambda+\beta) w_{+}^{p-1}\left(u_{0}-w_{+}\right)^{+} d z \quad \text { by } \boldsymbol{H}_{5}(\mathrm{ii}) .
\end{aligned}
$$

Since $A\left(w_{+}\right) \geq 0$ by hypothesis $\boldsymbol{H}_{\mathbf{5}}$ (ii), from the previous inequality we get

$$
\left\langle A\left(u_{0}\right)-A\left(w_{+}\right),\left(u_{0}-w_{+}\right)^{+}\right\rangle \leq \int_{\Omega}(\lambda+\beta)\left(w_{+}^{p-1}-u_{0}^{p-1}\right)\left(u_{0}-w_{+}\right)^{+} d z \leq 0,
$$

that is

$$
\int_{\left\{u_{0}>w_{+}\right\}}\left(\left|D u_{0}\right|_{N}^{p-2} D u_{0}-\left|D w_{+}\right|_{N}^{p-2} D w_{+}, D u_{0}-D w_{+}\right)_{N} d z \leq 0,
$$

so that $\mathscr{L}_{N}\left(\left\{u_{0}>w_{+}\right\}\right)=0$, i.e. $u_{0} \leq w_{+}$.

Therefore, we have $0 \leq u_{0} \leq w_{+}$, and so (5.6) becomes, by (5.1),

$$
A\left(u_{0}\right)+\beta u_{0}^{p-1}=N\left(u_{0}\right),
$$

with $N(u)(\cdot)=f(\cdot, u(\cdot))$ for all $u \in W_{n}^{1, p}(\Omega)$, that is

$$
\begin{cases}-\Delta_{p} u_{0}(z)+\beta(z) u_{0}^{p-1}=f\left(z, u_{0}(z)\right) & \text { a.e. in } \Omega \\ \frac{\partial u_{0}}{\partial n}=0 & \text { on } \partial \Omega\end{cases}
$$


Now, let $\xi_{r}>0$ be as in hypothesis $\boldsymbol{H}_{\mathbf{5}}(i i)$. We can always assume that $\xi_{r}>\|\beta\|_{\infty}$, and then we have

$$
-\Delta_{p} u_{0}(z)+\left(\xi_{r}+\beta(z)\right) u_{0}^{p-1}=f\left(z, u_{0}(z)\right)+\xi_{r} u_{0}(z)^{p-1} \geq 0 \quad \text { a.e. in } \Omega,
$$

and then

$$
\Delta_{p} u_{0}(z) \leq\left(\xi_{r}+\|\beta\|_{\infty}\right) u_{0}^{p-1} \quad \text { a.e. in } \Omega .
$$

By the Strong Maximum Principle (see Vazquez [42] and Pucci-Serrin [37]) the last inequality implies that $u_{0} \in \operatorname{int} C_{+}$.

Next, let $\delta>0$ and set $u_{\delta}=u_{0}+\delta \in \operatorname{int} C_{+}$. Then

$$
\begin{aligned}
& -\Delta_{p} u_{\delta}(z)+\left(\xi_{r}+\beta(z)\right) u_{\delta}(z)^{p-1} \\
= & -\Delta_{p} u_{0}(z)+\left(\xi_{r}+\beta(z)\right) u_{0}(z)^{p-1}+\rho(\delta) \text { with } \rho(\delta) \rightarrow 0^{+} \text {as } \delta \rightarrow 0^{+} \\
= & f\left(z, u_{0}(z)+\xi_{r} u_{0}(z)^{p-1}+\rho(\delta)\right. \\
\leq & f\left(z, w_{+}(z)\right)+\xi_{r} w_{+}(z)^{p-1}+\rho(\delta) \quad \text { a.e. in } \Omega
\end{aligned}
$$

by $\boldsymbol{H}_{\mathbf{5}}$ (ii) and the fact that $u_{0} \leq w_{+}$.

Let $\sigma=\underset{\Omega}{\operatorname{ess} \sup }\left\{f\left(\cdot, w_{+}(\cdot)\right)-\beta(\cdot) w_{+}(\cdot)^{p-1}\right\}<0$ by $\boldsymbol{H}_{\mathbf{5}}$ (ii). Since $\rho(\delta) \rightarrow$ $0^{+}$as $\delta \rightarrow 0^{+}$, for $\delta>0$ small we have that $\rho(\delta) \leq-\sigma$. Hence

$$
\rho(\delta)+f\left(z, w_{+}(z)\right) \leq \beta(z) w_{+}(z)^{p-1} \quad \text { a.e. in } \Omega,
$$

and by (5.8) we immediately get

$$
A\left(u_{\delta}\right)+\left(\xi_{r}+\beta\right) u_{\delta}^{p-1} \leq\left(\xi_{r}+\beta\right) w_{+}^{p-1} \leq A\left(w_{+}\right)+\left(\xi_{r}+\beta\right) w_{+}^{p-1}
$$

by $\boldsymbol{H}_{\mathbf{5}}$ (ii), and so

$$
A\left(u_{\delta}\right)-A\left(w_{+}\right)+\left(\xi_{r}+\beta\right)\left(u_{\delta}^{p-1}-w_{+}^{p-1}\right) \leq 0 .
$$

Acting on this inequality with $\left(u_{\delta}-w_{+}\right)^{+} \in W_{n}^{1, p}(\Omega)$, we immediately obtain $u_{\delta} \leq w_{+}$, and so $w_{+}-u_{0} \in \operatorname{int} C_{+}$. Recalling that $u_{0} \in \operatorname{int} C_{+}$, we can find $\rho>0$ so small that

$$
\begin{aligned}
\bar{B}_{n}^{C^{1}(\bar{\Omega})}\left(u_{0}\right) & =\left\{u \in C_{n}^{1}(\bar{\Omega}):\left\|u-u_{0}\right\|_{C_{n}^{1}(\bar{\Omega})} \leq \rho\right\} \\
& \subseteq\left[0, w_{+}\right]=\left\{u \in W_{n}^{1, p}(\Omega): 0 \leq u(z) \leq w_{+}(z) \text { a.e in } \Omega\right\} .
\end{aligned}
$$

Note that $\phi_{\lambda \mid\left[0, w_{+}\right]}^{+}=\phi_{\mid\left[0, w_{+}\right]}$. Therefore, $u_{0}$ is a local $C_{n}^{1}(\bar{\Omega})$-minimizer of $\phi$, and hence $u_{0}$ is a local $W_{n}^{1, p}(\Omega)$-minimizer of $\phi$ by Theorem 2.5 . 
To produce the negative smooth solution, we introduce the "negative" truncation-perturbation of $f$ defined by

$$
g_{\lambda}^{-}(z, x)= \begin{cases}f\left(z, w_{-}(z)\right)+\lambda\left|w_{-}(z)\right|^{p-2} w_{-}(z) & \text { if } x<w_{-}(z) \\ f(z, x)+\lambda|x|^{p-2} x & \text { if } w_{-}(z) \leq x \leq 0 \\ 0 & \text { if } x>0\end{cases}
$$

Of course, this is a Carathéodory function. Then, as natural, we set $G_{\lambda}^{-}(z, x)=$ $\int_{0}^{x} g_{\lambda}^{-}(z, s) d s$ and we consider the $C^{1}$ functional $\phi_{\lambda}^{-}: W_{n}^{1, p}(\Omega) \rightarrow \mathbb{R}$ defined by

$$
\phi_{\lambda}^{-}(u)=\frac{1}{p} \mathscr{E}(u)+\frac{\lambda}{p}\|u\|_{p}^{p}-\int_{\Omega} G_{\lambda}^{-}(z, u(z)) d z
$$

for all $u \in W_{n}^{1, p}(\Omega)$. Working as above, using this time (5.9) in place of (5.1), and $w_{-}$in place of $w_{+}$, we can produce a second smooth constant sign solution $v_{0} \in-\operatorname{int} C_{+}$with $v_{0}-w_{-} \in \operatorname{int} C_{+}$, which is a local minimizer of $\phi$.

The previous result can be immediately improved by using the tools recalled above.

Theorem 5.5. If hypotheses $\boldsymbol{H}_{5}$ hold and $\beta \in L^{\infty}(\Omega)$, then problem (4.1) has at least three nontrivial smooth solutions

$$
u_{0} \in \operatorname{int} C_{+}, \quad v_{0} \in-\operatorname{int} C_{+} \quad \text { and } \quad y_{0} \in C_{n}^{1}(\bar{\Omega}) \backslash\{0\} .
$$

Proof. From Proposition 5.4 we already have two constant sign smooth solutions $u_{0} \in \operatorname{int} C_{+}$and $v_{0} \in-\operatorname{int} C_{+}$. We also know that both are local minimizers of $\phi$, and without loss of generality we may assume that $\phi\left(v_{0}\right) \leq \phi\left(u_{0}\right)$. Now, let $\rho \in(0,1)$ be so small that

$$
\phi\left(v_{0}\right) \leq \phi\left(u_{0}\right)<\inf \left\{\phi(u):\left\|u-u_{0}\right\|=\rho\right\}=\eta_{\rho} .
$$

Let $D_{0}=\left\{v_{0}, u_{0}\right\}, D=\left\{u=\varepsilon u_{0}+(1-\varepsilon) v_{0}: 0 \leq \varepsilon \leq 1\right\}$ and $S=\partial B_{\rho}\left(u_{0}\right)$. It is easy to see that the pair $\left\{D_{0}, D\right\}$ is linking with $S$ in $W_{n}^{1, p}(\Omega)$. Also, since $\boldsymbol{H}_{5}$ (i), (ii) coincide with $\boldsymbol{H}_{\mathbf{1}}$ (i), (ii), Proposition 4.3 can be invoked, and thus $\phi$ satisfies the C-condition. In conclusion, we can apply Theorem 2.2 and find $y_{0} \in$ $W_{n}^{1, p}(\Omega)$ such that

$$
\phi^{\prime}\left(y_{0}\right)=0 \quad \text { and } \quad \phi\left(v_{0}\right) \leq \phi\left(u_{0}\right)<\eta_{\rho} \leq \phi\left(y_{0}\right)=\inf _{\gamma \in \Gamma \in[0,1]} \max _{t \in[} \phi(\gamma(t)),
$$

where $\Gamma=\left\{\gamma \in C\left([0,1], W_{n}^{1, p}(\Omega)\right): \gamma(0)=v_{0}, \gamma(1)=u_{0}\right\}$ and $\eta_{\rho}$ is defined in (5.10). 
From the second relation in (5.11), we see that $y_{0} \notin\left\{v_{0}, u_{0}\right\}$ and from [9, pages $84-85$, page 90 ] we know that (recall that $y_{0}$ is a critical point of mountain pass type)

$$
C_{1}\left(\phi, y_{0}\right) \neq 0 .
$$

On the other hand, recall that, by [24, Proposition 2.1], hypothesis $\boldsymbol{H}_{\mathbf{5}}$ (iii) implies that

$$
C_{k}(\phi, 0)=0 \quad \text { for all } k \geq 0 .
$$

Comparing (5.12) and (5.13), we infer that $y_{0} \neq 0$. Finally, from the equality in (5.11) we have that $y_{0}$ solves (4.1), and nonlinear regularity theory implies that $y_{0} \in C_{n}^{1}(\bar{\Omega})$.

In Theorem 5.5 the weight function $\beta$ is bounded, and this requirement appears to be essential in the proof. It is natural to ask wether we can have a multiplicity result when $\beta$ is unbounded. In this case, for the solutions we do not have regularity up to the boundary, and so we do not have at out disposal Theorem 2.5 relating local Sobolev and Hölder minimizers. For the case of unbounded $\beta$, we will prove a multiplicity theorem for coercive (hence nonresonant) problems.

Remark 5.6. We believe it is an interesting open problem wether one can have a multiplicity theorem for resonant problems in presence of an unbounded weight function $\beta$.

In light of the considerations just made, the hypotheses on the reaction $f$ now are the following:

$\boldsymbol{H}_{\mathbf{6}}: f: \Omega \times \mathbb{R} \rightarrow \mathbb{R}$ is a Carathéodory function such that $f(z, 0)=0$ a.e. in $\Omega$, hypothesis $\boldsymbol{H}_{\mathbf{6}}(\mathrm{i})$ is the same as the corresponding hypothesis $\boldsymbol{H}_{\mathbf{1}}(\mathrm{i})$ and

(ii): there exists $\theta \in L^{\infty}(\Omega)$ such that

$$
\theta(z) \leq \hat{\lambda}_{1} \quad \text { a.e. in } \Omega, \quad \theta \neq \hat{\lambda}_{1}
$$

and

$$
\lim _{x \rightarrow 0} \frac{p F(z, x)}{|x|^{p}} \leq \theta(z) \quad \text { uniformly for a.e. } z \in \Omega
$$

(iii): $\lim _{x \rightarrow 0} \frac{f(z, x)}{|x|^{p-2} x}=\lambda$ uniformly for a.e. $z \in \Omega$, with $\lambda>\hat{\lambda}_{2}$ and $\lambda \notin \sigma_{p}(\Omega)$.

Remark 5.7. Hypotheses $\boldsymbol{H}_{\mathbf{6}}$ (iii) is not very satisfactory, because from the point of view of the spectral properties developed in Section 3, it is not excluded the possibility that at least for some domains $\Omega$ we will have $\sigma_{p}=\left\{\hat{\lambda}_{1}\right\} \cup\left[\hat{\lambda}_{2}, \infty\right)$. We must underline that this is not the case if $p=2$ (see Section 6) and if $N=1$ (ordinary differential equations, see Zhang [43] and Binding-Rynne [6]). Nevertheless, such hypothesis has been already used in the literature (see, for example, Liu-Li [30] and Medeiros-Perera [33]. 
Example 5.8. The following function $f$ satisfies hypotheses $\boldsymbol{H}_{\mathbf{6}}$ :

$$
f(x)= \begin{cases}\lambda|x|^{p-2} x & \text { if }|x| \leq 1, \\ \theta|x|^{p-2} x+(\lambda-\theta)|x|^{q-2} x & \text { if }|x|>1\end{cases}
$$

with $\lambda>\hat{\lambda}_{2}, \lambda \notin \sigma_{p}(\Omega), \theta<\hat{\lambda}_{1}$ and $1<q<p$.

Let $\lambda>\hat{\lambda}_{2}, \lambda \notin \sigma_{p}(\Omega)$, and consider the $C^{1}$ functional $\psi_{\lambda}: W^{1, p}(\Omega) \rightarrow \mathbb{R}$ defined by

$$
\psi_{\lambda}(u)=\frac{1}{p} \mathscr{E}(u)-\frac{\lambda}{p}\|u\|_{p}^{p} \quad \text { for all } u \in W^{1, p}(\Omega) .
$$

Proposition 5.9. $C_{0}\left(\psi_{\lambda}, 0\right)=C_{1}\left(\psi_{\lambda}, 0\right)=0$.

Proof. Consider the open set $\mathcal{U}=\left\{u \in W^{1, p}(\Omega): \mathscr{E}(u)<\lambda\|u\|_{p}^{p}\right\}$. Clearly $\pm \hat{u}_{1} \in \mathcal{U}$.

We now show that $\mathcal{U}$ is path connected. To this end, let $u \in \mathcal{U}$ and let $V_{u}$ be the path component of $\mathcal{U}$ containing $u$ and set

$$
\eta_{u}=\inf \left\{\frac{\mathscr{E}(v)}{\|v\|_{p}^{p}}: v \in V_{u}\right\}<\lambda .
$$

For the previous optimization problem (5.14), we consider a minimizing sequence $\left\{u_{n}\right\}_{n \geq 1} \subseteq V_{u}$, and due to the $p$-homogeneity of $\mathscr{E}$, we can suppose that

$$
\left\|u_{n}\right\|_{p}=1 \text { for all } n \geq 1 \text { and } \mathscr{E}\left(u_{n}\right) \rightarrow \eta_{u} \text { as } n \rightarrow \infty \text {. }
$$

From (3.5) and (5.15) we immediately infer that $\left\{u_{n}\right\}_{n \geq 1} \subseteq W^{1, p}(\Omega)$ is bounded. So, passing to a suitable subsequence, if necessary, we may assume that

$$
u_{n} \rightarrow u \text { in } W^{1, p}(\Omega) \text { and } u_{n} \rightarrow u \text { in } L^{p q^{\prime}}(\Omega) \text { as } n \rightarrow \infty .
$$

Invoking the Ekeland variational principle and recalling that $M=\left\{v \in W^{1, p}(\Omega)\right.$ : $\left.\|v\|_{p}=1\right\}$, we can find a sequence $\left\{v_{n}\right\}_{n \geq 1} \subseteq \overline{V_{u} \cap M}$ such that

$$
\mathscr{E}\left(v_{n}\right) \leq \mathscr{E}\left(u_{n}\right) \leq \eta_{u}+\frac{1}{n^{2}}, \quad\left\|v_{n}-u_{n}\right\| \leq \frac{1}{n}
$$

and

$$
\mathscr{E}\left(v_{n}\right) \leq \mathscr{E}(v)+\frac{1}{n^{2}}\left\|v-v_{n}\right\| \quad \text { for all } v \in \overline{V_{u} \cap M} .
$$

If $v_{n} \in \partial\left(\overline{V_{u} \cap M}\right)$ for infinitely many $n$ 's, then from Lemma 3.5(c) of Cuesta-de Figueiredo-Gossez [13], for the related sequence of indexes, say for all $n \geq 1$, we have that

$$
\mathscr{E}\left(v_{n}\right)=\lambda \leq \mathscr{E}\left(u_{n}\right) \leq \eta_{u}+\frac{1}{n^{2}}<\lambda
$$


a contradiction. Therefore, $v_{n} \in V_{u} \cap M$ for all $n \geq 1$ and so, from (5.17), we get in a standard fashion

$$
\left(\mathscr{E}_{\mid M}\right)^{\prime}\left(v_{n}\right) \rightarrow 0 \quad \text { as } n \rightarrow \infty .
$$

From the proof of Proposition 3.6, we know that $\mathscr{E}_{\mid M}$ satisfies the PS-condition. Therefore, from (5.18) it follows that, at least for a subsequence, we have

$$
v_{n} \rightarrow v_{0} \quad \text { in } W^{1, p}(\Omega) \text { as } n \rightarrow \infty,
$$

and thus

$$
v_{0} \in \overline{V_{u} \cap M} \quad \text { and } \quad \mathscr{E}\left(v_{0}\right)=\eta_{u}<\lambda .
$$

As above, via Lemma 3.5(c) of [13], we infer that $v_{0} \in V_{u} \cap M$. Hence, in order to show the path-connectedness of $\mathcal{U}$, it suffices to join $\hat{u}_{1}$ and $v_{0}$ with a path staying in $\mathcal{U}$. If $v_{0} \leq 0$, then by virtue of Proposition 3.4, we have $v_{0}=-\hat{u}_{1}$, and then the desired path exists because of Proposition 3.6 and since $\lambda>\hat{\lambda}_{2}$. Analogously, if $v_{0} \geq 0$, then $v_{0}=\hat{u}_{1}$ and we are done. So, we may assume that $v_{0}^{ \pm} \neq 0$. Let

$$
y_{t}=\frac{v_{0}^{+}-(1-t) v_{0}^{-}}{\left\|v_{0}^{+}-(1-t) v_{0}^{-}\right\|_{p}} \in M \quad \text { for all } t \in[0,1] .
$$

By (5.18) and the strong convergence of $v_{n}$ to $v_{0}$, we know that

$$
\left\langle A\left(v_{0}\right), h\right\rangle+\int_{\Omega} \beta\left|v_{0}\right|^{p-2} v_{0} h d z=\eta_{u} \int_{\Omega}\left|v_{0}\right|^{p-2} v_{0} h d z \quad \text { for all } h \in W^{1, p}(\Omega) .
$$

In (5.19) first we choose $h=v_{0}^{+}$, and then $h=-v_{0}^{-}$, obtaining respectively

$$
\mathscr{E}\left(v_{0}^{+}\right)=\eta_{u}\left\|v_{0}^{+}\right\|_{p}^{p} \quad \text { and } \quad \mathscr{E}\left(v_{0}^{-}\right)=\eta_{u}\left\|v_{0}^{-}\right\|_{p}^{p}
$$

From these equalities and since the functions $v_{0}^{+}$and $v_{0}^{-}$have disjoint interior supports, we have

$$
\mathscr{E}\left(y_{t}\right)=\eta_{u}\left\|y_{t}\right\|_{p}^{p}=\eta_{u} \quad \text { for all } t \in[0,1],
$$

and thus $y_{t} \in \mathcal{U}$ for all $t \in[0,1]$.

Finally, note that $y_{0}=v_{0} /\left\|v_{0}\right\|_{p}=v_{0}$, since $v_{0} \in V_{u} \cap M$, and $y_{1}=$ $v_{0}^{+} /\left\|v_{0}^{+}\right\|_{p}=\hat{u}_{1}$ by Proposition 3.4. Therefore, the map $t \mapsto y_{t}$ is the desired path in $\mathcal{U}$ which joins $v_{0}$ and $\hat{u}_{1}$. This proves that $\mathcal{U}$ is path connected.

Now, let $z \in \mathcal{U}$. Then we have (for example, see [9, page 5])

$$
H_{0}(\mathcal{U}, z)=0 .
$$

The $p$-homogeneity of the functional $\psi_{\lambda}$ easily implies that the set

$$
\psi_{\lambda}^{0}=\left\{u \in W^{1, p}(\Omega): \psi_{\lambda}(u) \leq 0\right\}
$$


is contractible (it is enough to consider the segment $t u$ for any $u \in \psi_{\lambda}^{0}$ ). Hence (for example, see [21, page 389]),

$$
H_{k}\left(\psi_{\lambda}^{0}, z\right)=0 \quad \text { for all } k \geq 0 .
$$

The Second Deformation Lemma (see, for example, [18, page 628]), ensures that $\psi_{\lambda}^{0} \backslash\{0\}$ and $\psi_{\lambda}^{-\varepsilon}$ are homotopy equivalent for $\varepsilon>0$ small enough, since $u=0$ is the only critical point of $\psi_{\lambda}$. Similarly, if

$$
\dot{\psi}_{\lambda}^{0}=\left\{u \in W^{1, p}(\Omega): \psi_{\lambda}(u)<0\right\},
$$

then from Granas-Dugundji [21, page 407], we have that $\dot{\psi}_{\lambda}^{0}=\mathcal{U}$ and $\psi_{\lambda}^{-\varepsilon}$ are homotopy equivalent if $\varepsilon>0$ is small enough. Therefore, it follows that $\mathcal{U}$ and $\psi_{\lambda}^{0} \backslash\{0\}$ are homotopy equivalent, and so

$$
\begin{aligned}
& H_{k}(\mathcal{U}, z)=H_{k}\left(\psi_{\lambda}^{0} \backslash\{0\}, z\right) \quad \text { for all } k \geq 0, \\
& H_{0}\left(\psi_{\lambda}^{0} \backslash\{0\}, z\right)=0 \quad(\text { see }(5.20)) .
\end{aligned}
$$

Now, we consider the following reduced homology sequence:

$$
\begin{aligned}
\ldots H_{k}\left(\psi_{\lambda}^{0} \backslash\{0\}, z\right) \rightarrow & H_{k}\left(\psi_{\lambda}, z\right)=0 \\
& \stackrel{i_{*}}{\rightarrow} H_{k}\left(\psi_{\lambda}^{0}, \psi_{\lambda}^{0} \backslash\{0\}\right) \stackrel{\partial_{*}}{\rightarrow} H_{k-1}\left(\psi_{\lambda}^{0} \backslash\{0\}, z\right) \rightarrow \ldots,
\end{aligned}
$$

where $i_{*}$ is the group homomorphism arising from the inclusion map, and $\partial_{*}$ is the boundary homomorphism (for example, see Granas-Dugundji [21, page 388]). From (5.23), together with (5.21), we have

$$
\operatorname{ker} \partial_{*}=\operatorname{im} i_{*}=\{0\} .
$$

Thus $\partial_{*}$ is an isomorphism between $H_{k}\left(\psi_{\lambda}^{0}, \psi_{\lambda}^{0} \backslash\{0\}\right)$ and a subgroup of $H_{k-1}\left(\psi_{\lambda}^{0} \backslash\right.$ $\{0\}, z)$. Hence, by (5.22), and noting that $u=0$ is the only critical point of $\psi_{\lambda}$, we get

$$
C_{1}\left(\psi_{\lambda}, 0\right)=\boldsymbol{H}_{\mathbf{1}}\left(\psi_{\lambda}^{0}, \psi_{\lambda}^{0} \backslash\{0\}\right)=0 .
$$

This identity and (5.23) finally imply that

$$
C_{0}\left(\psi_{\lambda}, 0\right)=0,
$$

and the proposition is thus completely proved.

Using this proposition, we can now prove our multiplicity result for problem (4.1) when the weight function $\beta$ is unbounded.

Theorem 5.10. If hypotheses $\boldsymbol{H}_{\mathbf{6}}$ hold and $\beta \in L^{q}(\Omega)$ with $q>\frac{N p}{p-1}$, then problem (4.1) has at least three nontrivial smooth solutions $u_{0}, v_{0}, y_{0} \in C^{1, \alpha}(\Omega)(0<\alpha \leq 1)$ with

$$
v_{0}(z)<0<u_{0}(z) \quad \text { for all } z \in \Omega .
$$


Proof. Let $\hat{\beta}(z)=|\beta(z)|+1$, so that $\hat{\beta} \in L^{q}(\Omega)_{+}$, and consider the following truncation-perturbations of the reaction $f$ :

$$
\hat{f}_{+}(z, x)= \begin{cases}0 & \text { if } x \leq 0, \\ f(z, x)+\hat{\beta}(z) x^{p-1} & \text { if } x>0,\end{cases}
$$

and

$$
\hat{f}_{-}(z, x)= \begin{cases}f(z, x)+\hat{\beta}(z)|x|^{p-2} x & \text { if } x \leq 0, \\ 0 & \text { if } x>0 .\end{cases}
$$

Of course, both $\hat{f}_{ \pm}$are Carathéodory functions. Then we set $\hat{F}_{ \pm}(z, x)=\int_{0}^{x} \hat{f}_{ \pm}(z, s) d s$ and consider the $C^{1}$ functional $\hat{\phi}_{ \pm}: W^{1, p}(\Omega) \rightarrow \mathbb{R}$ defined by

$$
\hat{\phi}_{ \pm}(u)=\frac{1}{p} \mathscr{E}(u)+\frac{1}{p} \int_{\Omega} \hat{\beta}(z)|u(z)|^{p} d z-\int_{\Omega} \hat{F}_{ \pm}(z, u(z)) d z
$$

for all $u \in W^{1, p}(\Omega)$.

First we produce the positive solution $u_{0}$. By virtue of hypothesis $\boldsymbol{H}_{\mathbf{6}}(\mathrm{ii})$, given $\varepsilon>0$, there exists $c_{11}=c_{11}(\varepsilon)>0$ such that

$$
F(z, x) \leq \frac{1}{p}(\theta(z)+\varepsilon)|x|^{p}+c_{11} \quad \text { for all } x \in \mathbb{R} \text { and a.e. } z \in \Omega .
$$

Therefore, for $u \in W^{1, p}(\Omega)$ we have

$$
\begin{aligned}
\hat{\phi}_{+}(u) & =\frac{1}{p} \mathscr{E}(u)+\frac{1}{p} \int_{\Omega} \hat{\beta}(z)|u(z)|^{p} d z-\int_{\Omega} \hat{F}_{+}(z, u(z)) d z \\
& \geq \frac{1}{p} \mathscr{E}(u)+\frac{1}{p} \int_{\Omega} \hat{\beta}|u|^{p} d z-\frac{1}{p} \int_{\Omega} \theta\left(u^{+}\right)^{p} d z-\frac{\varepsilon}{p}\left\|u^{+}\right\|_{p}^{p}-c_{11} \mathscr{L}_{N}(\Omega)
\end{aligned}
$$

by (5.24) and (5.26). The last line of the previous inequality is

$$
\begin{aligned}
\geq & \frac{1}{p}\left[\mathscr{E}\left(u^{+}\right)-\int_{\Omega} \theta\left(u^{+}\right)^{p} d z\right]-\frac{\varepsilon}{p}\left\|u^{+}\right\|^{p} \\
& +\frac{1}{p}\left[\mathscr{E}\left(u^{-}\right)+\int_{\Omega} \hat{\beta}\left(u^{-}\right)^{p} d z\right]-c_{11} \mathscr{L}_{N}(\Omega) \\
\geq & \frac{\mu_{0}-\varepsilon}{p}\left\|u^{+}\right\|^{p}+\frac{1}{p}\left\|D u^{-}\right\|_{p}^{p} \\
& +\frac{1}{p} \int_{\Omega}(\hat{\beta}+\beta)\left(u^{-}\right)^{p} d z-c_{11} \mathscr{L}_{N}(\Omega)(\text { by Lemma 4.11) } \\
\geq & \frac{\mu_{0}-\varepsilon}{p}\left\|u^{+}\right\|^{p}+\frac{1}{p}\left\|D u^{-}\right\|_{p}^{p}+\frac{1}{p}\left\|u^{-}\right\|_{p}^{p}-c_{11} \mathscr{L}_{N}(\Omega),
\end{aligned}
$$

since $\hat{\beta}+\beta=2 \beta^{+}+1$. 
Choosing $\varepsilon \in\left(0, \mu_{0}\right)$, we see that

$$
\hat{\phi}_{+}(u) \geq c_{12}\|u\|^{p}-c_{11} \mathscr{L}_{N}(\Omega) \quad \text { for some } c_{12}>0,
$$

and so $\hat{\phi}_{+}$is coercive.

Moreover, it is easy to see that $\hat{\phi}_{+}$is sequentially weakly lower semicontinuous in $W^{1, p}(\Omega)$. Therefore, by the Weierstrass Theorem, we can find $u_{0} \in W^{1, p}(\Omega)$ such that

$$
\hat{\phi}_{+}\left(u_{0}\right)=\inf \left\{\hat{\phi}_{+}(u): u \in W^{1, p}(\Omega)\right\}=m_{+} .
$$

By virtue of hypothesis $\boldsymbol{H}_{\mathbf{6}}$ (iii), we can find $\delta_{0}>0$ such that

$$
F(z, x)>\frac{\hat{\lambda}_{1}}{p}|x|^{p} \text { for a.e. } z \in \Omega \text { and all } 0<|x| \leq \delta_{0} .
$$

Thus, if $t \in(0,1)$ is sufficiently small, we have $t\left\|\hat{u}_{1}\right\|_{\infty}<\delta_{0}$, and hence

$$
\begin{aligned}
\hat{\phi}_{+}\left(t \hat{u}_{1}\right) & =\frac{1}{p} \mathscr{E}\left(t \hat{u}_{1}\right)-\int_{\Omega} F\left(z, \hat{u}_{1}\right) d z \quad(\operatorname{see}(5.24)) \\
& \geq \int_{\Omega}\left(\frac{\hat{\lambda}_{1}}{p}\left(t \hat{u}_{1}\right)^{p}-F\left(z, t \hat{u}_{1}\right)\right) d z<0
\end{aligned}
$$

and so, recalling (5.27),

$$
\hat{\phi}\left(u_{0}\right)=\hat{m}_{+}<0=\hat{\phi}(0),
$$

i.e. $u_{0} \neq 0$.

From (5.27) we also have $\hat{\phi}_{+}^{\prime}\left(u_{0}\right)=0$, that is

$$
A\left(u_{0}\right)+(\beta+\hat{\beta})\left|u_{0}\right|^{p-2} u_{0}=\hat{N}_{+}\left(u_{0}\right),
$$

where $\hat{N}_{+}(u)(\cdot)=\hat{f}_{+}(\cdot, u(\cdot))$ for all $u \in W^{1, p}(\Omega)$.

On (5.28) we now act with $-u_{0}^{-} \in W^{1, p}(\Omega)$; by (5.24), and recalling that $\hat{\beta}+\beta \geq 1$, we immediately obtain

$$
\left\|D u_{0}^{-}\right\|_{p}^{p}+\left\|u_{0}^{-}\right\|_{p}^{p} \leq 0
$$

that is $u_{0}^{-} \equiv 0$, and so $u_{0} \geq 0, u_{0} \neq 0$.

Therefore (5.28) becomes

$$
A\left(u_{0}\right)+\beta u_{0}^{p-1}=N\left(u_{0}\right),
$$

with, by (5.24),

$$
N(u)(\cdot)=f(\cdot, u(\cdot)) \quad \text { for all } u \in W^{1, p}(\Omega) ;
$$


that is

$$
\begin{cases}-\Delta_{p} u_{0}(z)+\beta(z) u_{0}^{p-1}(z)=f\left(z, u_{0}(z)\right) & \text { a.e. in } \Omega \\ \frac{\partial u_{0}}{\partial n}=0 & \text { on } \partial \Omega\end{cases}
$$

i.e. $u_{0}$ solves problem (4.1), and, as usual, $u_{0} \in C^{1, \alpha}(\Omega)$ for some $\alpha \in(0,1]$.

Moreover, from the Harnack inequality of Trudinger [41] (see also Damascelli [14] and Pucci-Serrin [37]), we have that $u_{0}(z)>0$ for any $z \in \Omega$.

Next we show that $u_{0}$ is also a local minimizer for $\phi$. Thus, suppose that $u_{n} \rightarrow u_{0}$ in $W^{1, p}(\Omega)$ as $n \rightarrow \infty$. We will show that there exists $n_{0} \geq 1$ such that

$$
\phi\left(u_{n}\right) \geq \phi\left(u_{0}\right) \quad \text { for all } n \geq n_{0} .
$$

To this end, we write

$$
\begin{aligned}
\phi\left(u_{n}\right) & =\frac{1}{p}\left[\mathscr{E}\left(u_{n}^{+}\right)+\mathscr{E}\left(u_{n}^{-}\right)\right]-\int_{\Omega} F\left(z, u_{n}^{+}\right) d z-\int_{\Omega} F\left(z, u_{n}^{-}\right) d z \\
& \geq \phi\left(u_{n}^{+}\right)+\frac{1}{p} \mathscr{E}\left(u_{n}^{-}\right)-c_{13}\left\|u_{n}\right\|_{p q^{\prime}}^{p}
\end{aligned}
$$

for some $c_{13}>0$, thanks to Hölder's inequality and hypotheses $\boldsymbol{H}_{\mathbf{6}}(\mathrm{i})$, (iii).

Since $u_{0}>0$ and $u_{n} \rightarrow u_{0}$ in $W^{1, p}(\Omega)$, we have

$$
u_{n}^{+} \rightarrow u_{0}^{+} \quad \text { and } \quad u_{n}^{-} \rightarrow 0 \quad \text { as } n \rightarrow \infty
$$

If $u_{n}^{-}=0$ for all $n \geq n_{0}$, then $u_{n}^{+}=u_{n}$ for all $n \geq n_{0}$ and so (5.29) holds from (5.30). Thus, passing to a subsequence if necessary, we may assume that $u_{n}^{-} \neq 0$ for all $n \geq 1$. Now, for every $n \geq 1$, we set $\Omega_{n}^{-}=\left\{z \in \Omega: u_{n}(z)<0\right\}$, which has thus positive measure.

Claim 1: $\mathscr{L}_{N}\left(\Omega_{n}^{-}\right) \rightarrow 0$ as $n \rightarrow \infty$.

From the regularity of the Lebesgue measure on $\mathbb{R}^{N}$, we know that, given $\varepsilon>0$, we can find a compact set $K_{\varepsilon} \subset \Omega$ such that $\mathscr{L}_{N}\left(K_{\varepsilon}^{C}\right)=\mathscr{L}_{N}\left(\Omega \backslash K_{\varepsilon}\right) \leq \varepsilon$. From the very definition of $\Omega_{n}^{-}$we have

$$
\left\|u_{n}-u_{0}\right\|_{p}^{p}=\int_{\Omega}\left|u_{n}-u_{0}\right|^{p} d z \geq \int_{\Omega_{n} \cap K_{\varepsilon}}\left|u_{n}-u_{0}\right|^{p} d z \geq \int_{\Omega_{n} \cap K_{\varepsilon}}\left|u_{0}\right|^{p} d z
$$

and so

$$
\left\|u_{n}-u_{0}\right\|_{p}^{p} \geq \mu_{\varepsilon}^{p} \mathscr{L}_{N}\left(\Omega_{n}^{-} \cap K_{\varepsilon}\right),
$$

where $\mu_{\varepsilon}=\min \left\{u_{0}(z): z \in K_{\varepsilon}\right\}>0$.

Since $u_{n} \rightarrow u_{0}$ in $W^{1, p}(\Omega)$, in particular we have that $u_{n} \rightarrow u_{0}$ in $L^{p}(\Omega)$, and so from (5.31), we have

$$
\mathscr{L}_{N}\left(\Omega_{n}^{-} \cap K_{\varepsilon}\right) \rightarrow 0 \quad \text { as } n \rightarrow \infty
$$


On the other hand, note that

$$
\Omega_{n}^{-} \subseteq\left(\Omega_{n}^{-} \cap K_{\varepsilon}\right) \cup K_{\varepsilon}^{C}=\left(\Omega_{n}^{-} \cap K_{\varepsilon}\right) \cup\left(\Omega \backslash K_{\varepsilon}\right),
$$

and so

$\mathscr{L}_{N}\left(\Omega_{n}^{-}\right) \leq \mathscr{L}_{N}\left(\Omega_{n}^{-} \cap K_{\varepsilon}\right)+\mathscr{L}_{N}\left(\Omega \backslash K_{\varepsilon}\right) \leq \mathscr{L}_{N}\left(\Omega_{n}^{-} \cap K_{\varepsilon}\right)+\varepsilon \quad$ for all $n \geq 1 ;$

(5.32) now immediately implies that

$$
\limsup _{n \rightarrow \infty} \mathscr{L}_{N}\left(\Omega_{n}^{-}\right) \leq \varepsilon
$$

Since $\varepsilon>0$ is arbitrary, we can conclude that $\mathscr{L}_{N}\left(\Omega_{n}^{-}\right) \rightarrow 0$ as $n \rightarrow \infty$, and Claim 1 is thus proved.

Claim 2: there exists $n_{0} \geq 1$ such that

$$
\frac{1}{p} \mathscr{E}\left(u_{n}^{-}\right)>c_{13}\left\|u_{n}^{-}\right\|_{p q^{\prime}}^{p} \quad \text { for all } n \geq n_{0} .
$$

We argue by contradiction: so, suppose that, at least for a subsequence, we have

$$
\frac{1}{p} \mathscr{E}\left(u_{n}^{-}\right) \leq c_{13}\left\|u_{n}^{-}\right\|_{p q^{\prime}}^{p} \quad \text { for all } n \geq 1 .
$$

Set $y_{n}=\frac{u_{n}^{-}}{\left\|u_{n}^{-}\right\|_{p q^{\prime}}}, n \geq$. Then, using the homogeneity of $\mathscr{E}$, we get

$$
\frac{1}{p} \mathscr{E}\left(y_{n}\right) \leq c_{13} \quad \text { for all } n \geq 1,
$$

and so, by (3.5), we have that $\left\{y_{n}\right\}_{n \geq 1} \subseteq W^{1, p}(\Omega)$ is bounded. Hence, we may assume that

$$
y_{n} \rightarrow y \text { in } W^{1, p}(\Omega) \text { and } y_{n} \rightarrow y \text { in } L^{p q^{\prime}}(\Omega) \text { as } n \rightarrow \infty .
$$

Note that $\|y\|_{p q^{\prime}}=1$ and that $y \geq 0, y \neq 0$. Thus, we can find $\Lambda>0$ so small that, setting $\Omega_{\Lambda}=\{z \in \Omega: y(z) \geq \Lambda\}$, we have

$$
\mathscr{L}_{N}\left(\Omega_{\Lambda}\right)>0 .
$$

Then

$$
\begin{aligned}
\left\|y_{n}-y\right\|_{p}^{p} & =\int_{\Omega}\left|y_{n}-y\right|^{p} d z \geq \int_{\Omega_{\Lambda} \backslash \Omega_{n}^{-}}\left|y_{n}-y\right|^{p} d z \\
& \geq \int_{\Omega_{\Lambda} \backslash \Omega_{n}^{-}} y^{p} d z \quad\left(\text { by definition of } \Omega_{\Lambda}\right) \\
& \geq \Lambda^{p} \mathscr{L}_{N}\left(\Omega_{\Lambda} \backslash \Omega_{n}^{-}\right) \geq \Lambda^{p}\left(\mathscr{L}_{N}\left(\Omega_{\Lambda}\right)-\mathscr{L}_{N}\left(\Omega_{n}^{-}\right)\right) \quad \text { for all } n \geq 1,
\end{aligned}
$$


and so, by Claim 1 ,

$$
0=\lim _{n \rightarrow \infty}\left\|y_{n}-y\right\|_{p}^{p}=\Lambda^{p} \mathscr{L}_{N}\left(\Omega_{\Lambda}\right),
$$

which contradicts (5.33). This proves Claim 2.

Returning to (5.30), and using Claim 2, we obtain

$$
\phi\left(u_{n}\right) \geq \phi\left(u_{n}^{+}\right) \geq \phi\left(u_{0}\right) \quad \text { for all } n \geq n_{0},
$$

since $\phi_{\mid W_{+}}=\left(\hat{\phi}_{+}\right)_{\mid W_{+}}$, where $W_{+}=\left\{u \in W^{1, p}(\Omega): u \geq 0\right\}$, that is $u_{0}$ is a local minimizer for $\phi$.

Similarly, working with $\hat{\phi}_{-}$and using (5.25) in place of (5.24), we obtain a second constant sign smooth solution $v_{0}$ such that

$$
v_{0} \in C^{1, \alpha}(\Omega) \quad(0<\alpha \leq 1) \quad \text { and } v_{0}(z)<0 \quad \text { for all } z \in \Omega
$$

and such that $v_{0}$ is another local minimizer for $\phi$.

Having $u_{0}, v_{0}$ and assuming without any loss of generality that $\phi\left(v_{0}\right) \leq \phi\left(u_{0}\right)$, as in the proof of Theorem 5.5, via the use of Theorem 2.2, we can find a third critical point $y_{0}$ of $\phi$ such that $y_{0} \neq u_{0}, v_{0}$. Then $y_{0}$ solves problem (4.1) and, as usual, $y_{0} \in C^{1, \alpha}(\Omega)(0<\alpha \leq 1)$. Now we need to show that $y_{0} \neq 0$.

We consider the homotopy $h:[0,1] \times W^{1, p}(\Omega) \rightarrow W^{1, p}(\Omega)$ defined by

$$
h(t, u)=(1-t) \phi(u)+t \psi_{\lambda}(u) \quad \text { for all }(t, u) \in[0,1] \times W^{1, p}(\Omega),
$$

where $\psi_{\lambda}: W^{1, p}(\Omega) \rightarrow \mathbb{R}$ is the $C^{1}$ functional introduced in Proposition 5.9. We will show that $u=0$ is an isolated critical point of $h(t, \cdot)$ uniformly in $t \in[0,1]$. Arguing by contradiction, suppose we could find two sequences $\left\{t_{n}\right\}_{n \geq 1} \subseteq[0,1]$ and $\left\{u_{n}\right\}_{n \geq 1} \subseteq W^{1, p}(\Omega)$ such that

$$
\begin{aligned}
& t_{n} \rightarrow t \in[0,1], \quad u_{n} \rightarrow 0 \text { in } W^{1, p}(\Omega) \text { as } n \rightarrow \infty \\
& \text { and } h_{u}\left(t_{n}, u_{n}\right)=0 \text { for all } n \geq 1 .
\end{aligned}
$$

From the equation in (5.34), we have

$$
A\left(u_{n}\right)+\beta\left|u_{n}\right|^{p-2} u_{n}=\left(1-t_{n}\right) N\left(u_{n}\right)+t_{n} \lambda\left|u_{n}\right|^{p-2} u_{n} \quad \text { for all } n \geq 1,
$$

where, as above, $N(u)(\cdot)=f(\cdot, u(\cdot))$ for all $u \in W^{1, p}(\Omega)$. that

Let $y_{n}=\frac{u_{n}}{\left\|u_{n}\right\|}, n \geq 1$. Then $\left\|y_{n}\right\|=1$ for all $n \geq 1$ and so we may assume

$$
y_{n} \rightarrow y \text { in } W^{1, p}(\Omega) \text { and } y_{n} \rightarrow y \text { in } L^{p q^{\prime}}(\Omega) \text { as } n \rightarrow \infty .
$$

From (5.35) we obtain

$$
A\left(y_{n}\right)+\beta\left|y_{n}\right|^{p-2} y_{n}=\left(1-t_{n}\right) \frac{N\left(u_{n}\right)}{\left\|u_{n}\right\|^{p-1}}+t_{n} \lambda\left|y_{n}\right|^{p-2} y_{n} \quad \text { for all } n \geq 1 .
$$


On (5.37) we act with $y_{n}-y \in W^{1, p}(\Omega)$, pass to the limit as $n \rightarrow \infty$ and use (5.36), obtaining

$$
\lim _{n \rightarrow \infty}\left\langle A\left(y_{n}\right), y_{n}-y\right\rangle=0,
$$

which implies, with Proposition 2.7, that

$$
y_{n} \rightarrow y \text { in } W^{1, p}(\Omega), \text { and so }\|y\|=1 .
$$

Using hypothesis $\boldsymbol{H}_{\mathbf{6}}($ iii), we have

$$
\frac{N\left(u_{n}\right)}{\left\|u_{n}\right\|^{p-1}} \rightarrow \lambda|y|^{p-2} y \quad \text { in } L^{p^{\prime}}(\Omega) \text { as } n \rightarrow \infty .
$$

So, passing to the limit as $n \rightarrow \infty$ in (5.37) and using (5.38), we obtain

$$
A(y)+\beta|y|^{p-2} y=\lambda|y|^{p-2} y
$$

that is

$$
\begin{cases}-\Delta_{p} y(z)+\beta(z)|y(z)|^{p-2} y(z)=\lambda|y(z)|^{p-2} y(z) & \text { a.e. in } \Omega \\ \frac{\partial y}{\partial n}=0 & \text { on } \partial \Omega .\end{cases}
$$

Since $\lambda \notin \sigma_{p}(\Omega)$, from (5.40) we infer that $y=0$, which contradicts (5.38). Therefore, we can find $\rho \in(0,1)$ so small that $u=0$ is the only critical point in $\bar{B}_{\rho}=\left\{u \in W^{1, p}(\Omega):\|u\| \leq \rho\right\}$ of $h(t, \cdot)$ for all $t \in[0,1]$. Note that $h(0, \cdot)=\phi(\cdot)$, which is coercive due to hypothesis $\boldsymbol{H}_{\mathbf{6}}$ (ii) and Lemma 4.11 , hence it satisfies the PS-condition, while $h(1, \cdot)=\psi_{\lambda}(\cdot)$, and since $\lambda \notin \sigma_{p}(\Omega)$, also $\psi_{\lambda}$ satisfies the PS-condition. Thus, from the homotopy invariance of the critical groups (see Chang [10, page 334]), we have

$$
C_{k}(h(0, \cdot), 0)=C_{k}(h(1, \cdot), 0) \quad \text { for all } k \geq 0,
$$

that is

$$
C_{k}(\phi, 0)=C_{k}\left(\psi_{\lambda}, 0\right) \quad \text { for all } k \geq 0 .
$$

From Proposition 5.9, we have $C_{0}\left(\psi_{\lambda}, 0\right)=c_{1}\left(\psi_{\lambda}, 0\right)=0$, and so, from (5.41), we get

$$
C_{0}(\phi, 0)=C_{1}(\phi, 0)=0 .
$$

From Chang [9, pages 84-85] we know that

$$
C_{1}\left(\phi, y_{0}\right) \neq 0
$$

see also (5.12).

Comparing (5.42) and (5.43), we conclude that $y_{0} \neq 0$, as claimed. 


\section{Semilinear problems}

In this section we deal with semilinear problems (i.e., $p=2$ ), so the problem under consideration is now the following:

$$
\begin{cases}-\Delta u(z)+\beta(z) u(z)=f(z, u(z)) & \text { a.e. in } \Omega, \\ \frac{\partial u}{\partial n}=0 & \text { on } \partial \Omega .\end{cases}
$$

In this section we will assume that $\beta \in L^{\infty}(\Omega)$ and that $f(z, \cdot)$ is a $C^{1}$ function on $\mathbb{R}$. Using the tools of Morse theory, which is more effective since we work in the framework of Hilbert spaces, we are able to obtain stronger multiplicity results.

We start by considering the following linear eigenvalue problem:

$$
\begin{cases}-\Delta u(z)+\beta(z) u(z)=\hat{\lambda} u(z) & \text { a.e. in } \Omega \\ \frac{\partial u}{\partial n}=0 & \text { on } \partial \Omega .\end{cases}
$$

Suppose that $\beta \in L^{q}(\Omega)$ with $q>N / 2$. Then, from the Sobolev Embedding Theorem, we have that $u \in H^{1}(\Omega)$ implies $u \in L^{2 q^{\prime}}(\Omega)$. Also $\left|\int_{\Omega} \beta u^{2} d z\right| \leq$ $\|\beta\|_{q}\|u\|_{2 q^{\prime}}^{2}$. From (3.5) with $p=2$ we have

$$
\|u\|^{2} \leq \mathscr{E}(u)+c_{14}\|u\|_{2}^{2} \text { for some } c_{14}>0 \text { and all } u \in H^{1}(\Omega) .
$$

Therefore, by (6.3),

$$
\langle A(u), u\rangle+\int_{\Omega} \beta u^{2} d z=\|u\|^{2}+\int_{\Omega} \beta u^{2} d z=\mathscr{E}(u) \geq\|u\|^{2}-c_{14}\|u\|^{2}
$$

for all $u \in H^{1}(\Omega)$. Invoking Corollary 7.D, page 78, of Showalter [39], we see that problem (6.2) has a sequence $\left\{\hat{\lambda}_{n}\right\}_{n \geq 1}$ of distinct eigenvalues such that

$$
-c_{14}<\hat{\lambda}_{1}<\hat{\lambda}_{2} \leq \ldots \leq \hat{\lambda}_{n} \rightarrow \infty \quad \text { as } n \rightarrow \infty
$$

Moreover, we know that we can choose a corresponding sequence $\left\{\hat{u}_{n}\right\}_{n \geq 1} \subseteq$ $C^{1}(\Omega)$ of eigenfunctions which form an orthonormal basis for $L^{2}(\Omega)$ and an orthogonal basis for $H^{1}(\Omega)$. Again, only $\hat{\lambda}_{1}$ has constant sign eigenfunctions, while all the other eigenvalues have nodal eigenfunctions. Finally, if $\beta \in L^{\infty}(\Omega)$, then $\left\{\hat{u}_{n}\right\}_{n \geq 1} \subseteq C^{1}(\bar{\Omega})$.

We start with a multiplicity result for problems which are resonant at higher parts of the spectrum, i.e. with respect to eigenvalues $\hat{\lambda}>\hat{\lambda}_{2}$. Now, we do not require an oscillatory behaviour of $f(z, \cdot)$ (as in hypothesis $\boldsymbol{H}_{\mathbf{5}}(\mathrm{iii})$ ), and near the origin we do not have concave terms (as in hypothesis $\boldsymbol{H}_{\mathbf{5}}$ (iii)). Moreover, in order to exploit the full strength of Morse theory, we need a $C^{2}$ energy functional, which of course means that $f(z, \cdot)$ is of class $C^{1}$. More precisely, the new hypotheses on $f$ are:

$\boldsymbol{H}_{\mathbf{7}}: f: \Omega \times \mathbb{R} \rightarrow \mathbb{R}$ is a measurable function such that $f(z, 0)=0$ and $f(z, \cdot) \in$ $C^{1}(\mathbb{R})$ a.e. in $\Omega$, and 
(i): there exist $a \in L^{\infty}(\Omega)_{+}, c>0$ and $r \in\left[2,2^{*}\right)$ such that

$$
\left|f_{x}^{\prime}(z, x)\right| \leq a(z)+c|x|^{r-2} \quad \text { for a.e. } z \in \Omega \text { and all } x \in \mathbb{R} ;
$$

(ii): there exist an integer $m \geq 2, \eta_{0} \in L^{\infty}(\Omega), \eta_{0} \geq \hat{\lambda}_{m}$ a.e. in $\Omega, \eta_{0} \neq \lambda_{m}, \beta_{0}>0$ and $\tau \in(0,2)$ such that

$$
\eta_{0}(z) \leq \liminf _{|x| \rightarrow \infty} \frac{f(z, x)}{x} \leq \limsup _{|x| \rightarrow \infty} \frac{f(z, x)}{x} \leq \hat{\lambda}_{m+1} \text { uniformly for a.e. } z \in \Omega
$$

and

$$
\beta_{0} \leq \liminf _{|x| \rightarrow \infty} \frac{f(z, x) x-2 F(z, x)}{|x|^{\tau}} \quad \text { uniformly for a.e. } z \in \Omega
$$

(iii): $f_{x}^{\prime}(z, 0)=\lim _{x \rightarrow 0} \frac{f(z, x)}{x} \leq \hat{\lambda}_{1}$ uniformly for a.e. $z \in \Omega$ and $f_{x}(\cdot, 0) \neq \hat{\lambda}_{1}$.

Remark 6.1. Hypotheses $\boldsymbol{H}_{7}$ dictate a linear growth for $f(z, \cdot)$ both near zero and near $\pm \infty$. Moreover, hypothesis $\boldsymbol{H}_{7}$ (ii) permits resonance with an eigenvalue $\hat{\lambda}$ bigger than the first two, i.e. $\hat{\lambda} \notin\left\{\hat{\lambda}_{1}, \hat{\lambda}_{2}\right\}$.

Example 6.2. The following simple function $f$ satisfies hypotheses $\boldsymbol{H}_{7}$ :

$$
f(x)= \begin{cases}\theta x+\varepsilon x^{3} & \text { if }|x| \leq 1, \\ \eta x-3 \varepsilon x^{1 / 3} & \text { if }|x|>1,\end{cases}
$$

with $\eta<\hat{\lambda}_{1}, \eta \in\left(\hat{\lambda}_{m}, \hat{\lambda}_{m+1}\right], m \geq 2$, and $\varepsilon=\frac{1}{4}(\eta-\theta)>0$.

We will use the Sobolev space $H_{n}^{1}(\Omega)=\overline{C_{n}^{1}(\bar{\Omega})} \cdot \cdot \cdot \|$, for which we have the following orthogonal direct sum decomposition:

$$
H_{n}^{1}(\Omega)=\overline{\oplus_{i \geq 1} E\left(\hat{\lambda}_{i}\right)},
$$

where $E\left(\hat{\lambda}_{i}\right)$ denotes the eigenspace corresponding to the eigenvalue $\hat{\lambda}_{i}$. Recall that the eigenspaces $E\left(\hat{\lambda}_{i}\right), i \geq 1$, have the "unique continuation property" (UCP for short), namely: if $u \in E\left(\hat{\lambda}_{i}\right)$ vanishes on a set of positive measure, then $u(z)=0$ for all $z \in \bar{\Omega}$.

Now, let $\phi: H_{n}^{1}(\Omega) \rightarrow \mathbb{R}$ be the energy funtional associated to problem (6.1), defined by

$$
\phi(u)=\frac{1}{2} \mathscr{E}(u)-\int_{\Omega} F(z,(u)) d z \quad \text { for all } u \in H_{n}^{1}(\Omega) .
$$

Hypotheses $\boldsymbol{H}_{7}$ immediately imply that $\phi \in C^{2}\left(H_{n}^{1}(\Omega)\right)$. 
Proposition 6.3. If hypotheses $\boldsymbol{H}_{7}(\mathrm{i})$, (ii) hold and $\beta \in L^{\infty}(\Omega)$, then $\phi$ satisfies the $C$-condition.

Proof. Let $\left\{u_{n}\right\}_{n \geq 1} \subseteq H_{n}^{1}(\Omega)$ be a sequence such that

$$
\left|\phi\left(u_{n}\right)\right| \leq M_{12} \quad \text { for some } M_{12}>0 \text { and all } n \geq 1
$$

and

$$
\left(1+\left\|u_{n}\right\|\right) \phi^{\prime}\left(u_{n}\right) \rightarrow 0 \quad \text { in } H_{n}^{1}(\Omega)^{*} \text { as } n \rightarrow \infty .
$$

As in the proof of Proposition 4.3, from (6.4) and (6.5), we have

$$
\int_{\Omega}\left[f\left(z, u_{n}\right) u_{n}-2 F\left(z, u_{n}\right)\right] d z \leq M_{13} \quad \text { for some } M_{13}>0 \text { and all } n \geq 1
$$

which implies that

$$
\frac{1}{\left\|u_{n}\right\|^{\tau}} \int_{\Omega}\left[f\left(z, u_{n}\right) u_{n}-2 F\left(z, u_{n}\right)\right] d z \leq \frac{M_{13}}{\left\|u_{n}\right\|^{\tau}} \quad \text { for all } n \geq 1 \text {. }
$$

Claim: the sequence $\left\{u_{n}\right\}_{n \geq 1} \subseteq H_{n}^{1}(\Omega)$ is bounded.

Suppose by contradiction that the Claim is not true. Without any loss of generality, we may assume that $\left\|u_{n}\right\| \rightarrow \infty$ as $n \rightarrow \infty$. We set $y_{n}=u_{n} /\left\|u_{n}\right\|, n \geq 1$. Then $\left\|y_{n}\right\|=1$ for all $n \geq 1$, and so we can assume that

$$
y_{n} \rightarrow y \text { in } H_{n}^{1}(\Omega) \text { and } y_{n} \rightarrow y \text { in } L^{2}(\Omega) \text { as } n \rightarrow \infty \text {. }
$$

Thus, from (6.5), we immediately have

$$
\left|\left\langle A\left(y_{n}\right), h\right\rangle+\int_{\Omega} u_{n} h d z-\int_{\Omega} \frac{N\left(u_{n}\right)}{\left\|u_{n}\right\|} h d z\right| \leq \frac{\varepsilon_{n}\|h\|}{1+\left\|u_{n}\right\|} \quad \text { for all } h \in H_{n}^{1}(\Omega),
$$

with $\varepsilon_{n} \rightarrow 0$ as $n \rightarrow \infty$, and, as above, $N(u)(\cdot)=f(z, u(\cdot))$ for all $u \in H_{n}^{1}(\Omega)$. Hypotheses $\boldsymbol{H}_{7}(\mathrm{i})$, (ii) readily imply the existence of $\hat{a} \in L^{\infty}(\Omega)_{+}$and $\hat{c}>0$ such that

$$
|f(z, x)| \leq \hat{a}(z)+\hat{c}|x| \quad \text { for a.e. } z \in \Omega \text { and all } x \in \mathbb{R} \text {. }
$$

From (6.7) and (6.9) it follows that $\left\{\frac{N\left(u_{n}\right)}{\left\|u_{n}\right\|}\right\}_{n \geq 1} \subseteq L^{2}(\Omega)$ is bounded, and so we may assume that

$$
\frac{N\left(u_{n}\right)}{\left\|u_{n}\right\|} \rightarrow g \text { in } L^{2}(\Omega) \text { as } n \rightarrow \infty .
$$

Using hypothesis $\boldsymbol{H}_{7}$ (ii), we have that

$$
g=\hat{\eta} y \quad \text { with } \eta_{0}(z) \leq \hat{\eta}(z) \leq \lambda_{m+1} \text { a.e. in } \Omega \text {. }
$$


Now, recall that $A \in \mathscr{L}\left(H_{n}^{1}(\Omega), H_{n}^{1}(\Omega)^{*}\right)$. So, if in (3.11) we pass to the limit as $n \rightarrow \infty$ and use (6.7), (6.10) and (6.11), we obtain

$$
A(y)+\beta y=\hat{\eta} y .
$$

If $\hat{\eta} \neq \hat{\lambda}_{m+1}$, then acting on (6.12) with $y^{0}=\operatorname{proj}_{E\left(\hat{\lambda}_{m+1}\right)} y$, where $\operatorname{proj}_{E\left(\hat{\lambda}_{m+1}\right)} y$ is the orthogonal projection of $y$ onto the eigenspace $E\left(\hat{\lambda}_{m+1}\right)$; exploiting the orthogonality of the spaces $E\left(\hat{\lambda}_{i}\right)$, we have

$$
\left\|D y^{0}\right\|_{2}^{2}+\int_{\Omega} \beta\left(y^{0}\right)^{2} d z=\int_{\Omega} \hat{\eta}\left(y^{0}\right)^{2} d z
$$

and so

$$
\int_{\Omega}\left(\hat{\eta}(z)-\hat{\lambda}_{m+1}\right) y^{0}(z)^{2} d z=0 .
$$

On the other hand, by virtue of the UCP of $E\left(\hat{\lambda}_{m+1}\right)$ (see above), we have

$$
\int_{\Omega}\left(\hat{\eta}(z)-\hat{\lambda}_{m+1}\right) y^{0}(z)^{2} d z<0,
$$

which contradicts the previous identity.

If $\hat{\eta}=\hat{\lambda}_{m+1}$, then from (6.12) we have

$$
\begin{cases}-\Delta y+\beta y=\hat{\lambda}_{m+1} y & \text { a.e. in } \Omega, \\ \frac{\partial y}{\partial n}=0 & \text { on } \partial \Omega,\end{cases}
$$

that is $y \in E\left(\hat{\lambda}_{m+1}\right)$. Moreover, if in (6.8) we choose $h=y-y_{n} \in H_{n}^{1}(\Omega)$, pass to the limit as $n \rightarrow \infty$ and use (6.7), we have

$$
\lim _{n \rightarrow \infty}\left\langle A\left(y_{n}\right), y_{n}-y\right\rangle=0,
$$

hence $y_{n} \rightarrow y$ in $H_{n}^{1}(\Omega)$ by Proposition 2.7. Therefore, $y \in E\left(\hat{\lambda}_{m+1}\right) \backslash\{0\}$. By the UCP of $E\left(\hat{\lambda}_{m+1}\right)$, this implies that $|y(z)| \neq 0$ a.e. in $\Omega$, and then $\left|u_{n}(z)\right| \rightarrow \infty$ for a.e. $z \in \Omega$ as $n \rightarrow \infty$. Then, by virtue of hypothesis $\boldsymbol{H}_{7}$ (ii), we have

$$
\beta_{0} \leq \liminf _{n \rightarrow \infty} \frac{f\left(z, u_{n}(z)\right) u_{n}(z)-2 F\left(z, u_{n}(z)\right)}{\left|u_{n}(z)\right|^{\tau}} \quad \text { for a.e. } z \in \Omega .
$$

Hence, by Fatou's Lemma, we have

$$
\begin{aligned}
& \liminf _{n \rightarrow \infty} \frac{1}{\left\|u_{n}\right\|^{\tau}} \int_{\Omega}\left[f\left(z, u_{n}\right) u_{n}-2 F\left(z, u_{n}\right)\right] d z \\
= & \liminf _{n \rightarrow \infty} \int_{\Omega} \frac{f\left(z, u_{n}\right) u_{n}-2 F\left(z, u_{n}\right)}{\left|u_{n}\right|^{\tau}}\left|y_{n}\right|^{\tau} d z \\
\geq & \int_{\Omega} \liminf _{n \rightarrow \infty}\left(\frac{f\left(z, u_{n}\right) u_{n}-2 F\left(z, u_{n}\right)}{\left|u_{n}\right|^{\tau}}\left|y_{n}\right|^{\tau}\right) d z \\
\geq & \int_{\Omega} \beta_{0}|y|^{\tau} d z>0
\end{aligned}
$$


by (6.13) and (6.7). On the other hand, from (6.6) we have

$$
\limsup _{n \rightarrow \infty} \frac{1}{\left\|u_{n}\right\|^{\tau}} \int_{\Omega}\left[f\left(z, u_{n}\right) u_{n}-2 F\left(z, u_{n}\right)\right] d z \leq 0 .
$$

Comparing (6.14) and (6.15), we reach a contradiction and thus the Claim is proved.

By virtue of the Claim, we may assume that

$$
u_{n} \rightarrow u \text { in } H_{n}^{1}(\Omega) \text { and } u_{n} \rightarrow u \text { in } L^{2}(\Omega) .
$$

Then, choosing $h=y_{n}-y \in H_{n}^{1}(\Omega)$ in (6.8), passing to the limit as $n \rightarrow \infty$ and using as before (6.16) and Proposition 2.7, we conclude that $\phi$ satisfies the C-condition.

Next we compute the critical groups of $\phi$ at infinity.

Proposition 6.4. If hypotheses $\boldsymbol{H}_{7}$ (i), (ii) hold and $\beta \in L^{\infty}(\Omega)$, then $C_{k}(\phi, \infty)=$ $\delta_{k, d_{m}} \mathbb{Z}$ for all $k \geq 0$, where $d_{m}=\operatorname{dim} \oplus_{i=1}^{m} E\left(\hat{\lambda}_{i}\right) \geq 2$.

Proof. We fix $\lambda \in\left(\hat{\lambda}_{m}, \hat{\lambda}_{m+1}\right)$ and consider the $C^{1}$ functional $\psi_{\lambda}: H_{n}^{1}(\Omega) \rightarrow \mathbb{R}$ defined by

$$
\psi_{\lambda}(u)=\frac{1}{2} \mathscr{E}(u)-\frac{\lambda}{2}\|u\|_{2}^{2} \quad \text { for all } u i H_{n}^{1}(\Omega) .
$$

Now, we consider the homotopy $h:[0,1] \times H_{n}^{1}(\Omega) \rightarrow H_{n}^{1}(\Omega)$ defined by

$$
h(t, u)=(1-t) \phi(u)+t \psi_{\lambda}(u) \quad \text { for all }(t, u) \in[0,1] \times H_{n}^{1}(\Omega) .
$$

Evidently, $h(0, \cdot)=\phi, h(1, \cdot)=\psi_{\lambda}$ and both satisfy the C-condition (see Proposition (6.3) and recall that $\left.\lambda \notin \sigma_{2}(\Omega)\right)$.

Claim: There exist $\alpha \in \mathbb{R}$ and $\delta>0$ such that for every $(t, u) \in[0,1] \times H_{n}^{1}(\Omega)$ with $h(t, u) \leq \alpha$, there holds $(1+\|u\|)\left\|h_{u}(t, u)\right\|_{*} \geq \delta$.

We argue by contradiction and suppose the Claim is not true. Then we can find two sequences $\left\{t_{n}\right\}_{n \geq 1} \subseteq[0,1]$ and $\left\{u_{n}\right\}_{n \geq 1} \subseteq H_{n}^{1}(\Omega)$ such that

$$
\begin{gathered}
\qquad t_{n} \rightarrow[0,1], h\left(t_{n}, u_{n}\right) \rightarrow-\infty,\left\|u_{n}\right\| \rightarrow \infty \\
\text { and }\left(1+\left\|u_{n}\right\|\right) h_{u}(t, u) \rightarrow 0 \text { in } H_{n}^{1}(\Omega)^{*} \text { as } n \rightarrow \infty .
\end{gathered}
$$

From the last convergence in (6.17), after some rearrangements, we easily have

$$
\begin{aligned}
\mid\left\langle A\left(u_{n}\right), h\right\rangle & +\int_{\Omega} \beta u_{n} h d z-\left(1-t_{n}\right) \int_{\Omega} f\left(z, u_{n}\right) h d z-t_{n} \lambda \int_{\Omega} u_{n} h d z \mid \\
& \leq \frac{\varepsilon_{n}\|h\|}{1+\left\|u_{n}\right\|}
\end{aligned}
$$

for all $h \in H_{n}^{1}(\Omega)$ with $\varepsilon \rightarrow 0^{+}$as $n \rightarrow \infty$. 
Now, set $y_{n}=u_{n} /\left\|u_{n}\right\|, n \geq 1$. Then $\left\|y_{n}\right\|=1$ for all $n \geq 1$ and so we may assume that

$$
y_{n} \rightarrow y \text { in } H_{n}^{1}(\Omega) \text { and } y_{n} \rightarrow y \text { in } L^{2}(\Omega) .
$$

From (6.18) we have

$$
\begin{aligned}
& \left|\left\langle A\left(y_{n}\right), h\right\rangle+\int_{\Omega} \beta y_{n} h d z-\left(1-t_{n}\right) \int_{\Omega} \frac{f\left(z, u_{n}\right) h}{\left\|u_{n}\right\|} d z-t_{n} \lambda \int_{\Omega} y_{n} h d z\right| \\
& \quad \leq \frac{\varepsilon_{n}\|h\|}{\left(1+\left\|u_{n}\right\|\right)\left\|u_{n}\right\|}
\end{aligned}
$$

for all $h \in H_{n}^{1}(\Omega)$ and all $n \geq 1$.

From hypotheses $\boldsymbol{H}_{7}$ (i), (ii) it is clear that $\left\{\frac{f\left(\cdot, u_{n}(\cdot)\right)}{\left\|u_{n}\right\|}\right\}_{n \geq 1} \subseteq L^{2}(\Omega)$ is bounded, and so we may assume that

$$
\begin{aligned}
& \frac{f\left(\cdot, u_{n}(\cdot)\right)}{\left\|u_{n}\right\|} \rightarrow g \text { in } L^{2}(\Omega) \\
& \text { and } g=\hat{\eta} y, \text { with } \eta_{0}(z) \leq \hat{\eta}(z) \leq \lambda_{m+1} \text { a.e. in } \Omega,
\end{aligned}
$$

thanks to hypothesis $\boldsymbol{H}_{\mathbf{7}}$ (ii).

As before, if in (6.20) we choose $h=y_{n}-y \in H_{n}^{1}(\Omega)$ and pass to the limit as $n \rightarrow \infty$, then we end up with the fact that $y_{n} \rightarrow y$ in $H_{n}^{1}(\Omega)$ as $n \rightarrow \infty$ and $\|y\|=1$. have

If in (6.20) we pass to the limit as $n \rightarrow \infty$ and use (6.19) and (6.21), then we

$$
A(y)+\beta y=[(1-t) \hat{\eta}+t \lambda] y,
$$

that is

$$
\begin{cases}-\Delta y(z)+\beta(z) y(z)=[(1-t) \hat{\eta}(z)+t \lambda] y(z) & \text { a.e. in } \Omega \\ \frac{\partial y}{\partial n}=0 & \text { on } \partial \Omega .\end{cases}
$$

If $0<t \leq 1$, then

$$
\begin{cases}-\Delta y(z)+\beta(z) y(z)=\hat{\eta}_{t}(z) y(z) & \text { a.e. in } \Omega \\ \frac{\partial y}{\partial n}=0 & \text { on } \partial \Omega\end{cases}
$$

where we have set $\hat{\eta}_{t}(z)=(1-t) \hat{\eta}(z)+t \lambda$, and with $\hat{\lambda}_{m}<\hat{\eta}_{t}(z)<\lambda_{m+1}$ a.e. in $\Omega$, since $t>0$. From (6.23) and the UCP of the eigenspaces $E\left(\hat{\lambda}_{m}\right)$ and $E\left(\hat{\lambda}_{m+1}\right)$, we infer that $y=0$, a contradiction.

If $t=0$, then (6.22) becomes

$$
\begin{cases}-\Delta y(z)+\beta(z) y(z)=\hat{\eta}(z) y(z) & \text { a.e. in } \Omega \\ \frac{\partial y}{\partial n}=0 & \text { on } \partial \Omega .\end{cases}
$$


If $\hat{\eta} \not \equiv \hat{\lambda}_{m+1}$, then, as above, by virtue of the UCP of the eigenspaces, we have $y=0$, which is a contradiction.

If $\hat{\eta}=\hat{\lambda}_{m+1}$, then $y \in E\left(\hat{\lambda}_{m+1}\right)$ and so $y(z) \neq 0$ a.e. in $\Omega$ by the UCP of $E\left(\hat{\lambda}_{m+1}\right)$; as a consequence, $\left|u_{n}(z)\right| \rightarrow \infty$ for a.e. $z \in \Omega$ as $n \rightarrow \infty$. Then

$$
\beta_{0} \leq \liminf _{n \rightarrow \infty} \frac{f\left(z, u_{n}(z)\right) u_{n}(z)-2 F\left(z, u_{n}(z)\right)}{\left|u_{n}(z)\right|^{\tau}} \quad \text { for a.e. } z \in \Omega .
$$

From the second convergence in (6.17), we see that we can find an integer $n_{0} \geq 1$ such that

$$
\mathscr{E}\left(u_{n}\right)-\left(1-t_{n}\right) \int_{\Omega} 2 F\left(z, u_{n}\right) d z-t_{n} \lambda\left\|u_{n}\right\|_{2}^{2} \leq 0 \quad \text { for all } n \geq n_{0} .
$$

On the other hand, from (6.18) with $h=u_{n} \in H_{n}^{1}(\Omega)$, we see that, given $\varepsilon>0$, we can find $n_{1}=n_{1}(\varepsilon) \geq n_{0}$ such that

$$
-\mathscr{E}\left(u_{n}\right)+\left(1-t_{n}\right) \int_{\Omega} f\left(z, u_{n}\right) u_{n} d z+t_{n} \lambda\left\|u_{n}\right\|_{2}^{2} \leq \varepsilon \quad \text { for all } n \geq n_{1} .
$$

Recall that $t_{n} \rightarrow 0$, since we are considering the case $t=0$, so we may assume that $1-t_{n}>0$ for all $n \geq n_{1}$. Adding (6.25) and (6.26), we obtain

$$
\int_{\Omega}\left[f\left(z, u_{n}\right) u_{n}-2 F\left(z, u_{n}\right)\right] d z \leq \frac{\varepsilon}{1-t_{n}} \quad \text { for all } n \geq n_{1},
$$

and so

$$
\limsup _{n \rightarrow \infty} \frac{1}{\left\|u_{n}\right\|^{\tau}} \int_{\Omega}\left[f\left(z, u_{n}\right) u_{n}-2 F\left(z, u_{n}\right)\right] d z \leq 0 .
$$

$\mathrm{O}$ the other hand, from (6.24) and Fatou's Lemma, we have

$$
\begin{aligned}
& \liminf _{n \rightarrow \infty} \frac{1}{\left\|u_{n}\right\|^{\tau}} \int_{\Omega}\left[f\left(z, u_{n}\right) u_{n}-2 F\left(z, u_{n}\right)\right] d z \\
= & \liminf _{n \rightarrow \infty} \int_{\Omega} \frac{f\left(z, u_{n}\right) u_{n}-2 F\left(z, u_{n}\right)}{\left|u_{n}\right|^{\tau}}\left|y_{n}\right| \tau d z \\
\geq & \beta_{0} \int_{\Omega}|y|^{\tau} d z>0 .
\end{aligned}
$$

Comparing (6.27) and (6.28) we reach a contradiction, and this proves the Claim. have

The Claim permits us the use of Proposition 3.2 of Liang-Su [28], and so we

$$
C_{k}(h(0, \cdot), \infty)=C_{k}(h(1, \cdot), \infty) \quad \text { for all } k \geq 0,
$$

that is

$$
C_{k}(\phi, \infty)=C_{k}\left(\psi_{\lambda}, \infty\right) \quad \text { for all } k \geq 0
$$


Since $\lambda \notin \sigma_{2}(\Omega)$, the only critical point of $\psi_{\lambda}$ is $u=0$. Hence

$$
C_{k}\left(\psi_{\lambda}, \infty\right)=C_{k}\left(\psi_{\lambda}, 0\right) \quad \text { for all } k \geq 0 .
$$

But clearly $u=0$ is a nondegenerate critical point of $\psi_{\lambda}$ of Morse index $d_{m}=$ $\operatorname{dim} \oplus_{i=1}^{m} E\left(\hat{\lambda}_{i}\right) \geq 2$, since $m \geq 2$. Hence

$$
C_{k}\left(\psi_{\lambda}, 0\right)=\delta_{k, d_{m}} \mathbb{Z} \quad \text { for all } k \geq 0 .
$$

From (6.29), (6.30) and (6.31) we conclude that $C_{k}(\phi, \infty)=\delta_{k, d_{m}} \mathbb{Z}$ for all $k \geq 0$, as claimed.

Now we are ready for the first multiplicity result for the semilinear problem (6.1).

Theorem 6.5. If hypotheses $\boldsymbol{H}_{7}$ hold and $\beta \in L^{\infty}(\Omega)$, then problem (6.1) has at least three nontrivial smooth solutions

$$
u_{0} \in \operatorname{int} C_{+}, v_{0} \in-\operatorname{int} C_{+} \text {and } y_{0} \in C_{n}^{1}(\bar{\Omega}) \backslash\{0\} .
$$

Proof. Let $\lambda>\|\beta\|_{\infty}$, and consider the following truncation-perturbations of the reaction $f$ :

$$
f_{+}^{\lambda}(z, x)= \begin{cases}0 & \text { if } x \leq 0 \\ f(z, x)+\lambda x & \text { if } x>0\end{cases}
$$

and

$$
f_{-}^{\lambda}(z, x)= \begin{cases}f(z, x)+\lambda x & \text { if } x \leq 0, \\ 0 & \text { if } x>0 .\end{cases}
$$

Of course, both $f_{ \pm}^{\lambda}$ are Carathédodory functions. We introduce the natural functions $F_{ \pm}^{\lambda}(z, x)=\int_{0}^{x} f_{ \pm}^{\lambda}(z, s) d s$ and consider the functionals $\phi_{ \pm}^{\lambda}: H_{n}^{1}(\Omega) \rightarrow \mathbb{R}$ defined by

$$
\phi_{ \pm}^{\lambda}(u)=\frac{1}{2} \mathscr{E}(u)+\frac{\lambda}{2}\|u\|_{2}^{2}-\int_{\Omega} F_{ \pm}^{\lambda}(z, u) d z \quad \text { for all } u \in H_{n}^{1}(\Omega) .
$$

Evidently $\phi_{ \pm}^{\lambda}$ is of class $C^{2}$ in $H_{n}^{1}(\Omega)$, with the only possible exception of the point $u=0$. that

By virtue of hypothesis $\boldsymbol{H}_{7}(i i i)$, given $\varepsilon>0$, we can find $\delta=\delta(\varepsilon)>0$ such

$$
F(z, x) \leq \frac{1}{2}\left(\theta_{0}+\varepsilon\right) x^{2} \quad \text { for a.e. } z \in \Omega \text { and all }|x| \leq \delta,
$$

where we have set $\theta_{0}(\cdot)=f_{x}^{\prime}(\cdot, 0)$. 
Let $u \in C_{n}^{1}(\bar{\Omega})$ be such that $\|u\|_{C_{n}^{1}(\bar{\Omega})} \leq \delta$. Then we have

$$
\begin{aligned}
\phi_{+}^{\lambda}(u) & =\frac{1}{2} \mathscr{E}(u)+\frac{\lambda}{2}\|u\|^{2}-\int_{\{u \geq 0\}} F(z, u) d z-\frac{\lambda}{2} \int_{\{u \geq 0\}} u^{2} d z \\
& \geq \frac{1}{2} \mathscr{E}(u)-\frac{1}{2} \int_{\{u \geq 0\}} \theta_{0} u^{2} d z+\frac{\lambda}{2} \int_{\Omega}\left(u^{+}\right)^{2} d z-\frac{\varepsilon}{2} \int_{\Omega} u^{2} d z \\
& \geq \frac{1}{2} \mathscr{E}(u)-\frac{1}{2} \int_{\Omega} \theta_{0} u^{2} d z-\frac{\varepsilon}{2}\|u\|^{2} \quad \text { (by (6.34)) } \\
& \geq \frac{\mu_{0}-\varepsilon}{2}\|u\|^{2} \quad \text { (by Lemma 4.11). }
\end{aligned}
$$

Choosing $\varepsilon \in\left(0, \mu_{0}\right)$, from (6.35) we see that

$$
\phi_{+}^{\lambda}(u) \geq 0=\phi_{+}^{\lambda}(0) \quad \text { for all } u \in C_{n}^{1}(\bar{\Omega}) \text { with }\|u\|_{C_{n}^{1}(\bar{\Omega})} \leq \delta,
$$

and then $u=0$ is a local minimizer of $\phi_{+}^{\lambda}$ (see Theorem 2.5).

We may always assume that $u=0$ is an isolated critical point of $\phi_{+}^{\lambda}$. Indeed, if this is not the case, we can find a sequence $\left\{u_{n}\right\}_{n \geq 1} \subseteq H_{n}^{1}(\Omega)$ such that $u_{n} \rightarrow 0$ in $H_{n}^{1}(\Omega)$ as $n \rightarrow \infty$ and

$$
\left(\phi_{+}^{\lambda}\right)^{\prime}\left(u_{n}\right)=0 \quad \text { for all } n \geq 1,
$$

that is

$$
A\left(u_{n}\right)+(\beta+\lambda) u_{n}=N_{+}^{\lambda}\left(u_{n}\right),
$$

where $N_{+}^{\lambda}(u)(\cdot)=f_{+}^{\lambda}(\cdot, u(\cdot))$ for all $u \in H_{n}^{1}(\Omega)$.

In (6.35) we act with $-u_{n}^{-} \in H_{n}^{1}(\Omega)$. Thanks to (6.32) we thus have

$$
\mathscr{E}\left(u_{n}^{-}\right)+\lambda\left\|u_{n}^{-}\right\|_{2}^{2}=0,
$$

and so, recalling that $\lambda>\|\beta\|_{\infty}$, we get $\left\|u_{n}^{-}\right\|=0$, and hence $u_{n} \geq 0, u_{n} \neq 0$, for all $n \geq 1$.

Then (6.36) becomes

$$
A\left(u_{n}\right)+\beta u_{n}=N\left(u_{n}\right) \quad \text { for all } n \geq 1,
$$

where $N(u)(\cdot)=f(\cdot, u(\cdot))$ for all $u \in H_{n}^{1}(\Omega)$. Thus $u \in C_{+}$solves (6.1) for all $n \geq 1$, and by the maximum principle of Vazquez [42], $u_{n} \in \operatorname{int} C_{+}$for all $n \geq 1$.

So we have produced a whole sequence of nontrivial smooth solutions of (6.1) which are strictly positive in $\Omega$, and the first statement of Theorem 6.5 is obviously proved. 
Therefore, we assume that $u=0$ is isolated; thus we can find $\rho \in(0,1)$ so small that

$$
\phi_{+}^{\lambda}(0)=0<\inf \left\{\phi_{+}^{\lambda}(u):\|u\|=\rho\right\}=\eta_{+}^{\lambda} .
$$

Hypothesis $\boldsymbol{H}_{7}$ (ii) implies that

$$
\phi_{+}^{\lambda}\left(t \hat{u}_{1}\right) \rightarrow-\infty \quad \text { as } t \rightarrow \infty .
$$

A slight modification of the proof of Proposition 6.3 shows that $\phi_{+}^{\lambda}$ satisfies the Ccondition (recall that only the principal eigenvalue $\hat{\lambda}_{1}$ has constant sign eigenfunctions). Thus, this fact, together with (6.37) and (6.38), permit the use of Theorem 2.3 (the Mountain Pass Theorem). Hence we can find $u_{0} \in H_{n}^{1}(\Omega)$ such that

$$
\phi_{+}^{\lambda}(0)=0<\eta_{+}^{\lambda} \leq \phi_{+}^{\lambda}\left(u_{0}\right) \text { and }\left(\phi_{+}^{\lambda}\right)^{\prime}\left(u_{0}\right)=0 .
$$

From (6.39) it follows that $u_{0} \neq 0$, and acting as done from (6.36) for $u_{n}$, we get that $u_{0} \in C_{+} \backslash\{0\}$ and solves (6.1).

Since $f(z, \cdot) \in C^{1}(\mathbb{R})$, by using hypothesis $\boldsymbol{H}_{7}(i)$ we see that for every $r>0$ we can find $\xi_{r}>0$ such that for a.e. $z \in \Omega$ the function $x \mapsto f(z, x)+\xi_{r} x$ is nondecreasing on $[0, r]$. Let us choose $r=\left\|u_{0}\right\|_{\infty}$. Then

$$
-\Delta u_{0}(z)+\left(\beta(z)+\xi_{r}\right) u_{0}(z)=f\left(z, u_{0}(z)\right)+\xi_{r} u_{0}(z) \quad \text { a.e. in } \Omega,
$$

and so

$$
\Delta u_{0}(z) \leq\left(\|\beta\|_{\infty}+\xi_{r}\right) u_{0}(z) \quad \text { a.e. in } \Omega .
$$

As usual, an application of the Strong Maximum Principle of Vazquez [42], implies that $u_{0} \in \operatorname{int} C_{+}$.

Since $u_{0}$ is a critical point of mountain pass type, as above, we have

$$
C_{k}\left(\phi_{+}^{\lambda}, u_{0}\right)=\delta_{k, 1} \mathbb{Z} \quad \text { for all } k \geq 0,
$$

see Chang [10].

Now, recall that $u_{0} \in \operatorname{int} C_{+}$and note that $\phi_{+\mid C_{+}}^{\lambda}=\phi_{\mid C_{+}}$. Hence

$$
C_{k}\left(\phi_{+\mid C_{n}^{1}(\bar{\Omega})}^{\lambda}, u_{0}\right)=C_{k}\left(\phi_{\mid C_{n}^{1}(\bar{\Omega})}, u_{0}\right) \quad \text { for all } k \geq 0 .
$$

But from Bartsch [4] and Liu-Wu [31] we know that

$$
C_{k}\left(\phi_{+\mid C_{n}^{1}(\bar{\Omega})}^{\lambda}, u_{0}\right)=C_{k}\left(\phi_{+}^{\lambda}, u_{0}\right) \quad \text { for all } k \geq 0
$$

and

$$
C_{k}\left(\phi_{\mid C_{n}^{1}(\bar{\Omega})}, u_{0}\right)=C_{k}\left(\phi, u_{0}\right) \quad \text { for all } k \geq 0 .
$$

From (6.40), (6.41), (6.42) and (6.43) it follows that

$$
C_{k}\left(\phi, u_{0}\right)=\delta_{k, 1} \mathbb{Z} \quad \text { for all } k \geq 0 .
$$


Similarly, working with the functional $\phi_{-}^{\lambda}$ and using this time (6.33) in place of (6.32), we produce another constant sign smooth solution $v_{0} \in-\operatorname{int} C_{+}$such that

$$
C_{k}\left(\phi, v_{0}\right)=\delta_{k, 1} \mathbb{Z} \quad \text { for all } k \geq 0 .
$$

Since $u=0$ is a local minimizer of $\phi$, we have

$$
C_{k}(\phi, 0)=\delta_{k, 0} \mathbb{Z} \quad \text { for all } k \geq 0 .
$$

From Proposition 6.4 we know that $C_{k}(\phi, \infty)=\delta_{k, d_{m}} \mathbb{Z}$ for all $k \geq 0$. This means that there is $y_{0} \in H_{n}^{1}(\Omega)$, a critical point of $\phi$, such that

$$
C_{d_{m}}\left(\phi, y_{0}\right) \neq 0 \text {. }
$$

Since $d_{m} \geq 2$, comparing (6.47) with (6.44), (6.45) and (6.46), we infer that $y_{0} \notin$ $\left\{0 u_{0}, v_{0}\right\}$. Moreover, by regularity theory, $y_{0} \in C_{n}^{1}(\bar{\Omega})$ and it solves problem (6.1).

Remark 6.6. A natural question is whether it is possible to relax the assumption on $\beta$, and to have the previous result when $\beta \in L^{q}(\Omega)$ with $q>N / 2$. Indeed, if we were dealing with the Dirichlet problem, then this would have been the case. Because then, by a bootstrap technique, we have that the solutions belong to $C_{0}^{1}(\bar{\Omega})$, so they are smooth up to the boundary (see Struwe [40, pages 270-271]). For the Neumann problem, this is no longer true. So our proof, with the application of Theorem 2.5, fails, and we do not know if the multiplicity theorem is true when $\beta \in L^{q}(\Omega)$ with $q>N / 2$.

Finally, we have the "semilinear" version of Theorem 5.5, producing this time four nontrivial smooth solutions for problem (6.1).

The hypotheses on $f$ now are:

$\boldsymbol{H}_{\mathbf{8}}: f: \Omega \times \mathbb{R} \rightarrow \mathbb{R}$ is a measurable function such that $f(z, 0)=0$ and $f(z, \cdot) \in$ $C^{1}(\mathbb{R})$ a.e. in $\Omega$, hypothesis $\boldsymbol{H}_{\mathbf{8}}(i)$ is the same as the corresponding hypothesis $\boldsymbol{H}_{7}(\mathrm{i})$ and

(ii): there exist $\eta_{0} \in L^{\infty}(\Omega), \eta_{0} \geq \hat{\lambda}_{1}$ a.e. in $\Omega, \eta_{0} \neq \lambda_{1}, \beta_{0}>0$ and $\tau \in[1,2)$ such that $\eta_{0}(z) \leq \liminf _{|x| \rightarrow \infty} \frac{2 F(z, x)}{x^{2}} \leq \limsup _{|x| \rightarrow \infty} \frac{2 F(z, x)}{x^{2}}<\hat{\lambda}_{2} \quad$ uniformly for a.e. $z \in \Omega$ and

$$
\beta_{0} \leq \liminf _{|x| \rightarrow \infty} \frac{f(z, x) x-2 F(z, x)}{|x|^{\tau}} \quad \text { uniformly for a.e. } z \in \Omega
$$

(iii): there exist $\delta_{0}>0$ and $\mu \in(1,2)$ such that $\mu F(z, x) \geq f(z, x) x>0$ a.e. in $\Omega$ for all $0<|x| \leq \delta_{0}$. Moreover, there exist $K>0$ such that $F(z, x) \geq K|x|^{\mu}$ a.e. in $\Omega$ for all $|x| \leq \delta_{0}$; 
(iv): there exist two functions $\left.w_{ \pm} \in H^{1}(\Omega) \cap C^{(} \bar{\Omega}\right)$ such that

$$
\begin{gathered}
w_{-}(z) \leq c_{-}<0<c_{+} \leq w_{+} \quad \text { for all } z \in \bar{\Omega}, \\
\underset{\Omega}{\operatorname{ess} \sup }\left\{f\left(\cdot, w_{+}(\cdot)\right)-\beta(\cdot) w_{+}(\cdot)\right\}<0<\underset{\Omega}{\operatorname{essinf}}\left\{f\left(\cdot, w_{-}(\cdot)\right)-\beta(\cdot) w_{-}(\cdot)\right\}, \\
A\left(w_{-}\right) \leq 0 \leq A\left(w_{+}\right) \quad \text { in } H_{n}^{1}(\Omega)^{*} .
\end{gathered}
$$

Remark 6.7. The reader will recognize that $\boldsymbol{H}_{\mathbf{8}}$ (iii) is simply $\boldsymbol{H}_{\mathbf{1}}$ (iii) $=\boldsymbol{H}_{\mathbf{5}}$ (iii) with $p=2$, while $\boldsymbol{H}_{\mathbf{8}}$ (iv) coincides with $\boldsymbol{H}_{\mathbf{5}}$ (ii) when $p=2$.

Example 6.8. The following function satisfies hypotheses $\boldsymbol{H}_{\mathbf{8}}$ :

$$
f(x)= \begin{cases}\lambda x^{1 / 6}-\eta x^{1 / 3} & \text { if }|x| \leq 1 \\ \eta_{0} x-\theta & \text { if }|x|>1\end{cases}
$$

with $\eta_{0} \in\left(\hat{\lambda}_{1}, \hat{\lambda}_{2}\right], \lambda, \eta>0, \eta-\lambda>\|\beta\|_{\infty}$ and $\theta=\frac{2}{3} \eta-\frac{5}{6} \lambda>0$.

Concerning problem (6.1), we have the following final multiplicity result.

Theorem 6.9. If hypotheses $\boldsymbol{H}_{\mathbf{8}}$ hold and $\beta \in L^{\infty}(\Omega)$, then problem (6.1) has at least four nontrivial smooth solutions

$$
u_{0} \in \operatorname{int} C_{+}, v_{0} \in-\operatorname{int} C_{+}, y_{0}, \hat{y} \in C_{n}^{1}(\bar{\Omega}) .
$$

Proof. From Theorem 5.5 with $p=2$, which can be applied thanks to Remark 6.1, we already have three nontrivial smooth solutions

$$
u_{0} \in \operatorname{int} C_{+}, v_{0} \in-\operatorname{int} C_{+} \text {and } y_{0} \in C_{n}^{1}(\bar{\Omega}) .
$$

By Proposition 5.4 applied with $p=2$, we know that the two constant sign solutions $u_{0}$ and $v_{0}$ are local minimizers of $\phi$. Hence, by (2.2),

$$
C_{k}\left(\phi, u_{0}\right)=C_{k}\left(\phi, v_{0}\right)=\delta_{k, 0} \mathbb{Z} \quad \text { for all } k \geq 0 .
$$

Moreover, $y_{0}$ is a critical point of mountain pass type (see the proof of Theorem 5.5), and since $\phi \in C^{2}\left(H_{n}^{1}(\Omega)\right)$, we have

$$
C_{k}\left(\phi, y_{0}\right)=\delta_{k, 1} \mathbb{Z} \quad \text { for all } k \geq 0,
$$

see Bartsch [4].

Also, recall that hypothesis $\boldsymbol{H}_{\mathbf{8}}$ (iii) implies that

$$
C_{k}(\phi, 0)=0 \quad \text { for all } k \geq 0 .
$$

Finally, it is not hard to see that an analogous of Proposition 6.4 holds with $d_{m}=1$, being $m=1$ and $E\left(\hat{\lambda}_{1}\right)=\mathbb{R}$; and from such a result we immediately have

$$
C_{k}(\phi, \infty)=\delta_{k, 1} \mathbb{Z} \quad \text { for all } k \geq 0 .
$$


Now, suppose by contradiction that $\left\{0, u_{0} v_{0}\right\}$ are the only critical points of $\phi$. Using (6.48), (6.49), (6.50), (6.51) and the Morse relation (2.1), we obtain

$$
2(-1)^{0}+(-1)^{1}=(-1)^{1},
$$

a contradiction. This means that $\phi$ has one more critical point $\hat{y} \notin\left\{0, u_{0} v_{0}\right\}$. Then, as usual, $\hat{y} \in C_{n}^{1}(\bar{\Omega})$ and it solves problem (6.1).

\section{References}

[1] S. Aizicovici, N. Papageorgiou and V. Staicu, Existence of multiple solutions with precise sign information for superlinear Neumann problems, Ann. Mat. Pura Appl. (4) 188 (2009), 679-719.

[2] W. Allegretto and Y.X. Huang, A Picone's identity for the p-Laplacian and applications, Nonlinear Anal. 32 (1998), 819-830.

[3] P. BARTOlo, V. BENCI and D. ForTUnATO, Abstract critical point theorems and applications to some nonlinear problems with "strong" resonance at infinity, Nonlinear Anal. 7 (1983), 981-1012.

[4] T. BARTSCH, Critical point theory on partially ordered Hilbert spaces, J. Funct. Anal. 186 (2001), 117-152.

[5] T. BARTSCH and S. LI, Critical point theory for asymptotically quadratic functionals and applications to problems with resonance, Nonlinear Anal. 28 (1997), 419-441.

[6] P. A. BINDING and B. P. RYNNE, Variational and non-variational eigenvalues of the $p$ Laplacian, J. Differential Equations 244 (2008), 24-39.

[7] I. BIRINDELLI and F. DEMENGEL, Existence of solutions for semi-linear equations involving the p-Laplacian: the non coercive case, Calc. Var. Partial Differential Equations 20 (2004), 343-366.

[8] H. Brezis and L. Nirenberg, $H^{1}$ versus $C^{1}$ local minimizers, C. R. Acad. Sci. Paris Sér. I Math. 317 (1993), 465-472.

[9] K.-C. Chang, "Infinite-dimensional Morse Theory and Multiple Solution Problems", In: Progress in Nonlinear Differential Equations and their Applications 6, Birkhäuser Boston, MA, 1993.

[10] K.-C. CHANG, "Methods in Nonlinear Analysis", Springer Monographs in Mathematics, Springer-Verlag, Berlin, 2005.

[11] M. CUESTA, Eigenvalue problems for the p-Laplacian with indefinite weights, Electron. J. Differential Equations 2001, No. 33, 1-9.

[12] M. CUeSTA and H. RAMOS QUOIRIN, A weighted eigenvalue problem for the p-Laplacian plus a potential, NoDEA Nonlinear Differential Equations Appl. 16 (2009), 469-491.

[13] M. CuestA, D. DE Figueiredo and J. P. Gossez, The beginning of the Fučik spectrum for the p-Laplacian, J. Differential Equations 159 (1999), 212-238.

[14] L. DAMASCELLI, Comparison theorems for some quasilinear degenerate elliptic operators and applications to symmetry and monotonicity results, Ann. Inst. H. Poincaré Anal. Non Linéaire 15 (1998), 493-516.

[15] L. M Del PEZzo, J. Fernández Bonder and J. D. Rossi, An optimization problem for the first weighted eigenvalue problem plus a potential, Proc. Amer. Math. Soc. 138 (2010), $3551-3567$.

[16] J. Fernández Bonder and L. M. Del Pezzo, An optimization problem for the first eigenvalue of the p-Laplacian plus a potential, Commun. Pure Appl. Anal. 5 (2006), 675690 . 
[17] J. P. García Azorero, I. Peral Alonso and J. J. Manfredi, Sobolev versus Hölder local minimizers and global multiplicity for some quasilinear elliptic equations, Commun. Contemp. Math. 2 (2000), 385-404.

[18] L. Gasinski and N. S. PApageorgiou, "Nonlinear analysis", Series in Mathematical Analysis and Applications 9, Chapman \& Hall/CRC, Boca Raton, FL, 2006.

[19] N. Ghoussoub, "Duality and Perturbation Methods in Critical Point Theory", Cambridge Tracts in Mathematics 107, Cambridge University Press, Cambridge, 1993.

[20] S. GoldberG, "Unbounded Linear Operators. Theory and Applications", McGraw-Hill Book Co., New York, 1966.

[21] A. GRanas and J. Dugundu, "Fixed Point Theory", Springer Monographs in Mathematics, Springer-Verlag, New York, 2003.

[22] Z. GUO and Z. ZHANG, $W^{1, p}$ versus $C^{1}$ local minimizers and multiplicity results for quasilinear elliptic equations, J. Math. Anal. Appl. 286 (2003), 32-50.

[23] S. HU and N. S. PAPAgEORgIOU, Nonlinear Neumann equations driven by a nonhomogeneous differential operator, Commun. Pure Appl. Anal. in press.

[24] Q. JIU and J. SU, Existence and multiplicity results for perturbations of the p-Laplacian, J. Math. Anal. Appl. 281 (2003), 587-601.

[25] A. LÊ, Eigenvalue problems for the p-Laplacian, Nonlinear Anal. 64 (2006), 1057-1099.

[26] L. LEADI and A. YeCHOUI, Principal eigenvalue in an unbounded domain with indefinite potential, NoDEA Nonlinear Differential Equations Appl. 17 (2010), 391-409.

[27] C. LI, The existence of infinitely many solutions of a class of nonlinear elliptic equations with Neumann boundary condition for both resonance and oscillation problems, Nonlinear Anal. 54 (2003), 431-443.

[28] Z. LIANG and J. SU, Multiple solutions for semilinear elliptic boundary value problems with double resonance, J. Math. Anal. Appl. 354 (2009), 147-158.

[29] G. M. LIEBERMAN, Boundary regularity for solutions of degenerate elliptic equations, Nonlinear Anal. 12 (1988), 1203-1219.

[30] S. LIU and S. LI, Existence of solutions for asymptotically 'linear' p-Laplacian equations, Bull. London Math. Soc. 36 (2004), 81-87.

[31] J. LIU and S. WU, Calculating critical groups of solutions for elliptic problem with jumping nonlinearity, Nonlinear Anal. 49 (2002), 779-797.

[32] J. LóPEZ-GómEZ, The maximum principle and the existence of principal eigenvalues for some linear weighted boundary value problems, J. Differential Equations 127 (1996), 263294.

[33] E. Medeiros and K. Perera, Multiplicity of solutions for a quasilinear elliptic problem via the cohomological index, Nonlinear Anal. 71 (2009), 3654-3660.

[34] D. Motreanu, V. Motreanu and N.S. PAPAgeorgiou, Nonlinear Neumann problems near resonance, Indiana Univ. Math. J. 58 (2009), 1257-1279.

[35] D. MugnaI, Addendum to: Multiplicity of critical points in presence of a linking: application to a superlinear boundary value problem, NoDEA Nonlinear Differential Equations Appl. 11 (2004), 379-391, and a comment on the generalized Ambrosetti-Rabinowitz condition, NoDEA Nonlinear Differential Equations Appl. 19 (2004), 299-301.

[36] N. S. PAPAgeORgiou and S. T. Kyritsi, "Handbook of Applied Analysis", Advances in Mechanics and Mathematics 19, Springer, New York, 2009.

[37] P. PuCCI and J. SERrin, "The Maximum Principle", Progress in Nonlinear Differential Equations and their Applications 73, Birkhäuser Verlag, Basel, 2007.

[38] A. QIAN, Existence of infinitely many nodal solutions for a superlinear Neumann boundary value problem, Bound. Value Probl. 2005, 329-335.

[39] R. E. Showalter, “ Hilbert Space Methods for Partial Differential Equations”, Monographs and Studies in Mathematics 1, Pitman, London, 1977.

[40] M. Struwe, "Variational Methods. Applications to Nonlinear Partial Differential Equations and Hamiltonian Systems", Fourth edition, Springer-Verlag, Berlin, 2008. 
[41] N. S. TRudinger, On Harnack type inequalities and their application to quasilinear elliptic equations, Comm. Pure Appl. Math. 20 (1967), 721-747.

[42] J. VAZQUEZ, A strong maximum principle for some quasilinear elliptic equations, Appl. Math. Optim. 12 (1984), 191-202.

[43] M. ZHANG, The rotation number approach to eigenvalues of the one-dimensional pLaplacian with periodic potentials, J. London Math. Soc. (2) 64 (2001), 125-143.

Dipartimento di Matematica

e Informatica

Università di Perugia

Via Vanvitelli, 1

06123 Perugia, Italia

mugnai@dipmat.unipg.it

Department of Mathematics

National Technical University

Zografou Campus

Athens 15780, Greece

npapg@math.ntua.gr 